


\section{Dis-locating innovation: \\ Amphibious geographies of creative reuse and alternative value production}

Iulian I.V. Barba Lata 


\section{Thesis committee}

\section{Promotor}

Prof. Dr C. Minca

Professor of Cultural Geography

Wageningen University \& Research

\section{Co-promotor}

Dr M. Duineveld

Assistant professor, Cultural Geography Group

Wageningen University \& Research

\section{Other members}

Prof. Dr B.E. Buscher, Wageningen University \& Research

Prof. Dr M. Jones, University of Sheffield, UK

Prof. Dr H. Lorimer, University of Glasgow, UK

Prof. Dr P.M. Macnaghten, Wageningen University \& Research

This research was conducted under the auspices of the Graduate School of Social Sciences (WASS). 


\title{
Dis-locating innovation: Amphibious geographies of creative reuse and alternative value production
}

\author{
Iulian I.V. Barba Lata
}

\section{Thesis}

submitted in fulfilment of the requirements for the degree of doctor at Wageningen University by the authority of the Rector Magnificus

Prof. Dr A.P.J. Mol, in the presence of the

Thesis Committee appointed by the Academic Board to be defended in public on Wednesday 1 February 2017 at 4 p.m. in the Aula 
Iulian I.V. Barba Lata

Dis-locating innovation: Amphibious geographies of creative reuse and alternative value production, 141 pages.

PhD thesis, Wageningen University \& Research, Wageningen, NL (2017)

With references, with summary in English

ISBN 978-94-6343-013-5

DOI $10.18174 / 396514$ 
To all silent Others that enable our meaningful lives... 


\section{Acknowledgements}

The process of bringing a $\mathrm{PhD}$ to completion is very much a nonlinear one, as I have discovered over these past years. When I started, thought that great enthusiasm and persistence would suffice to move mountains. It took a while to realise that a good deal of 'engineering' and pragmatism is also required for that kind of dislocation work. And, as rewarding as the latter might occasionally prove both professionally and personally, it is nonetheless part of an eminently painful metamorphosis, one that constantly redraws the confines of your comfort bubble. Having reached this point, perhaps the best way to describe this phase of my life journey would be as a bittersweet symphony. Fortunately, many wonderful people stood by me in this endeavour, offering support during the most difficult moments and sharing the joy of small victories along the way. Though far from perfect, this dissertation would not have been anywhere near its final shape without the vibrant ecology that endorsed it.

I am indebted to my supervisors Claudio Minca and Martijn Duineveld for their invaluable guidance and friendship. After the years spent on turning a rather unconventional research project into a graspable end product, it would be about time to admit that both of them excelled in coping with my caprices, stubbornness and often obscure ideas. Moreover, they proved surprisingly innovative in doing so, through salient counter-examples of creative reuse and constantly putting the squeeze on my endless exercises of anticipation. While I certainly had difficulties in identifying the immediate benefits, on the longer run they became more obvious. In turn, besides the inherent training in scholarly gymnastics, our teamwork provided a much needed learning experience, particularly in cultivating my patience and humbleness. I consider myself really lucky to have had the chance of working with you and digest the complexity of academic life by your side. Claudio, Martijn, what a ride this has been!

Being a junior scholar comes with all sorts of advantages, including the fact that one could end up having several academic parents. Right from an early stage, I had the privilege of meeting bright scholars and get inspired by them. I am grateful to Rosi Braidotti, Mike Crang and Gunnar Olsson, people I respect enormously for their work, as well as their generosity. Special thanks go to Annemarie Mol and colleagues from the Health, Care and the Body group at the University of Amsterdam, for all the therapy and food for thought provided during the walking seminars. My work has benefitted greatly from the many interesting conversations I had over time with Sebastian Abrahamsson, Kristof van Assche, Raoul Beunen, Maartje Bulkens, Clemens Driessen, Martijn Felder, Paolo Giaccaria, Henk de Haan, Alan Latham, Hamzah Muzaini, Meghann Ormond, Arjaan Pellis, Lauren Wagner and Arjen 
Zegwaard. The person I owe the most for my PhD trajectory is Christian Abrahamsson. Chris, you have been a close friend and constant source of inspiration. The memorable discussions and long strolls around Uppsala and Lund are still very fresh in my mind.

Although I enjoy traveling, it often happens to get homesick. During the past five years or so, the Cultural Geography group offered me that warm and comfy home to long for. And what made it special, was the GEO family. I am proud to have been part of it, and truly thankful for all the great moments we have spent together. Undoubtedly, the best place to start my tour is the infamous room B301. Back in 2012, I took over from Meike and Katharina in sharing the office with my dear Maartje and Arjaan. With such pleasant and fun people it was not that difficult to live up to the legacy of B301 as the hottest spot in the group. Later on, Alexandra and Rodrigo have joined the cause, and just before I left, Eugenie also moved in. In contrast to my early days in the group, GEO now accommodates a spirited $\mathrm{PhD}$ community and it has been a pleasure to witness its development over the years. I would like to thank Alexandra, Arjaan, Chalermpat, Eugenie, John, Maartje, Martijn, Nelly, Nowella, Pieternel, Rita, Rodrigo, Said, Tabitha, Trista and Zulkhairi, with whom I shared the ups and downs of PhD life. As most of them, I was fortunate to benefit from the kind support of Chin Ee, Clemens, Hamzah, Henk, Karin, Lauren, Maarten, Michael, Meghann and Rene, who were always there to offer advice and encouragement. I owe a big thanks as well to Carla, Keen and Mieke for being such a nice company and frequently performing wonders with even the most tangled admin issues. GEO family, wish you all the best, and keep going strong wherever the road takes you!

Another place close to my heart is de Leeuwenborch, home base of the Wageningen School of Social Sciences (WASS). My journey would have felt incomplete without the time spent there as a member of the WASS PhD council. That proved a rewarding experience in so many regards. Carolina, Geralda, Gohar Jannette, Jeroen and Muriel: thank you for making teamwork so fun and being such dedicated comrades!

What I find most remarkable about the process of doing research are the unexpected synergies one ends up joggling with. I am grateful to quite a few people for enabling that to happen, particularly to Maxim Amosov, Rutger de Graaf, Femke Haccoû, Sergey Ivanov, Anastasia Juritsjeva, Vladimir Kolossov, Keira Medvedeva, Erwin van Tuijl, William Veerbeek and Theodoor van der Voort. I am much obliged also to my former supervisors Liviu Ianasi and Paolo Russo, as well as to Luis Carvalho, for encouraging me to pursue an academic trajectory. On the same note, I would like to thank Leonie Janssen-Jansen, Marleen Buizer, Katrien Termeer and Henk Wolfert for entrusting me with a new mission.

Caught in the swirl of my project, I have failed many times to manage the work routine in a decent manner. Thankfully, I had plenty of friends around to constantly 
remind me there are all sorts of fun things happening outside the $\mathrm{PhD}$ sphere. This goes to Arjaan, Hamzah, Martijn, Maartje, Chin Ee, Lauren, Meghann and Oscar, Miha and Gabriel, Arjen, Jeroen, Liz, Koos and Regina, Jan-Pieter and Stacy, Koos and Jose, Ilias, Evangelos, Raoul, Joop, Alexandru and Viorel.

Last but not least, I dedicate this dissertation to my family: to my parents Petre and Elena for all the wisdom, support and love I have been blessed with, and for inspiring me to acknowledge that when the going gets tough, the tough get going; to Silviu and Marian for being the best brothers in the world; to Catalina and Constantin for being so caring and cheering up even the darkest days; and most of all, to Ioana, my love and better restless half. You are the bright fire in my life, the sea, the clear sky and all the things that make me feel complete! 


\section{Table of contents}

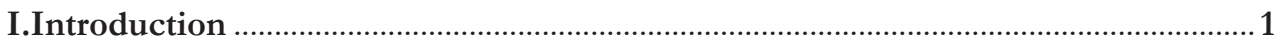

I.1. Objective and research questions …........................................................................... 4

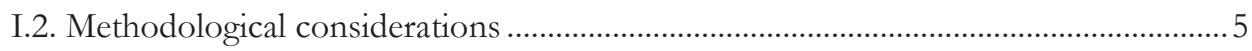

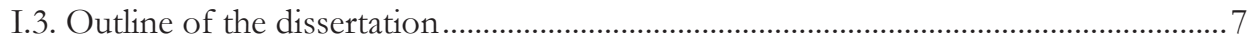

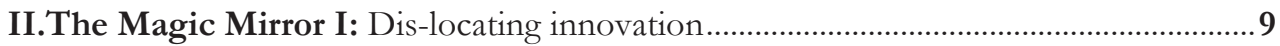

II.1. Performative dimensions of innovation: the road to creative destruction ....................11

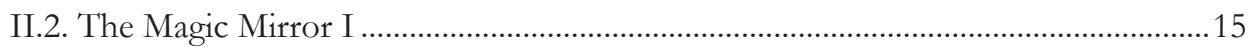

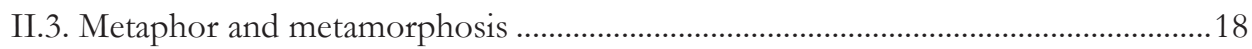

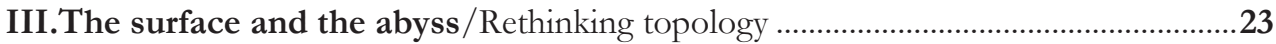

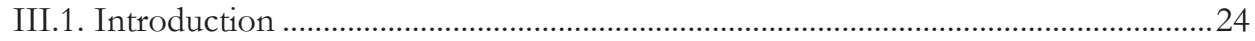

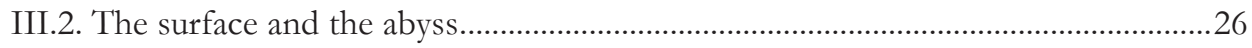

III.3. Topological trajectories: from the mathematics of space to the space of mathematics....31

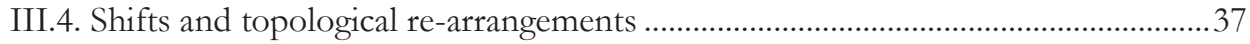

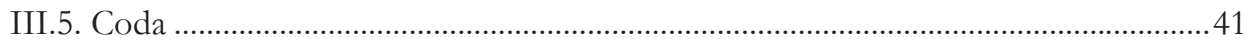

IV.The floating churches of Volgograd: River topologies and warped spatialities of

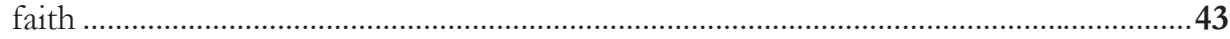

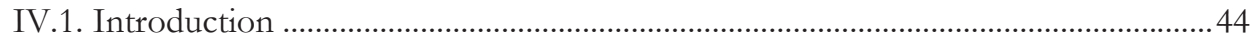

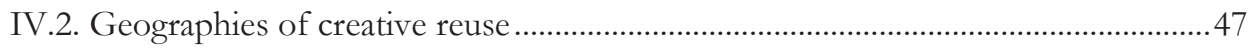

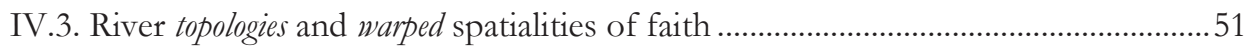

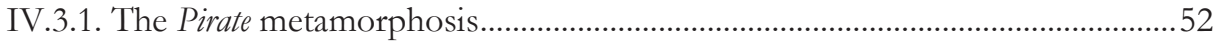

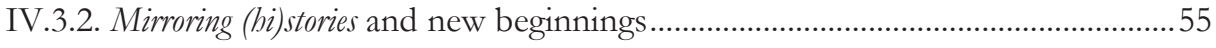

IV.3.3. Saint Nicholas return: the repair vessel, the barge and the landing craft...........57

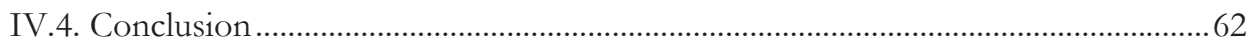


V. A harbour on land: De Ceuvel's amphibious topologies of creative reuse

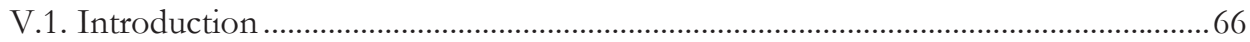

V.2. Alternatives of creative reuse: reanimating urban materialities .................................69

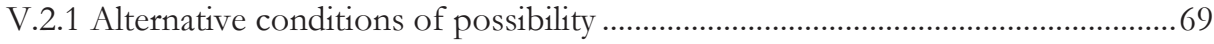

V.2.2 Reanimating urban materialities ............................................................................. 71

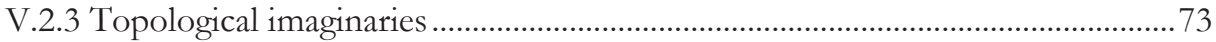

V.3. De Ceuvel: on-land harbour and fluid sociotechnical arrangements ...................... 75

V.3.1 Creative reuse imaginaries and ad hoc coalitions ................................................. 75

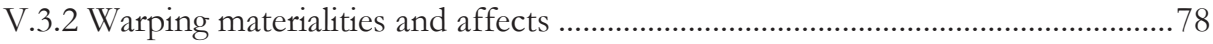

V.4. Topologies of creative reuse: transient fixed points and shifting spatialities .....................83

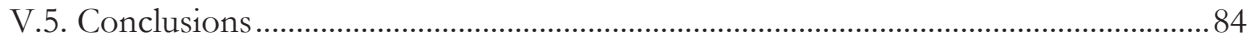

VI.The Magic Mirror II: Floating churches, stranded houseboats and other surprising (re)turns

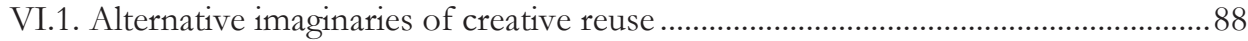

VI.2. Topologies of fixed points and shifting spatialities ................................................. 91

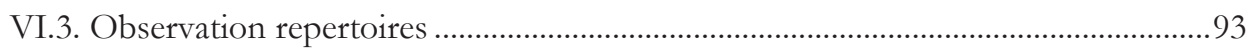

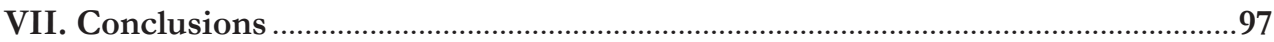

VII.1. Navigating realms of loose connections ................................................................

VII.2. Warping spatiotemporal conditions of encounter..............................................100

VII.3. The dance of contingent imaginaries and anamorphic reflections ......................101

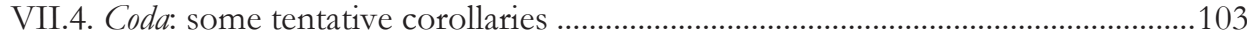

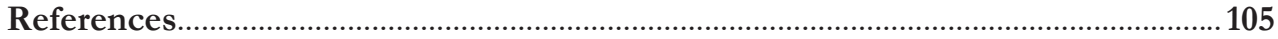

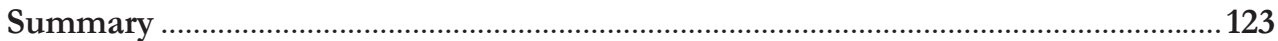




\section{Introduction}

"Knowledge is always accompanied with accessories of emotion and purpose."

(Whitehead, 1967:4)

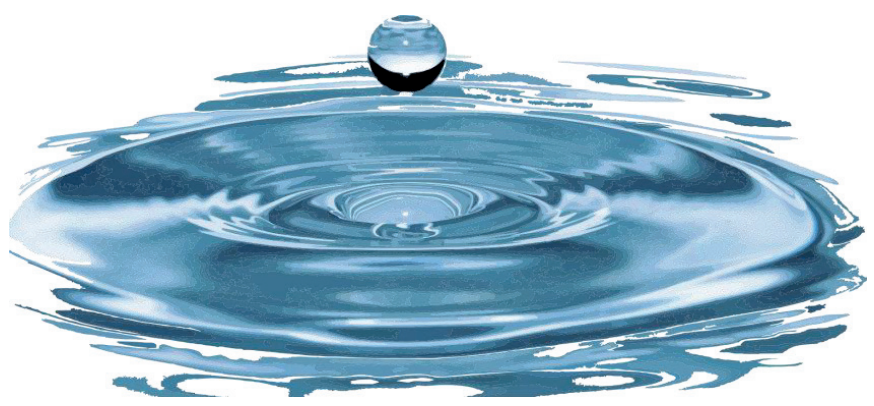




\section{Introduction}

Innovation is a hot topic nowadays in both academia and societal debates. The upheaval of information and communication technologies during the 1990's turned out to be the tipping point of a new cultural economy, animated by emergent forms of knowledge production and symbolic capital (Amin and Thrift, 2007; Scott, 1999). The fundamental changes occasioned by this 'epochal shift', which some commentators heralded as marking a transition from industrial to "immaterial or biopolitical production" (Hardt, 2010:18), have kept scholars of various denominations busy ever since. Resulting turns in economic theory and beyond flagged the liberalization of research and development activities as a new strategy to generate competitive advantage (Chesbrough, 2003; von Hippel, 2005), which fostered a greater emphasis on aspects related to creativity and the engineering of effective environments for innovation. In order to capture the creative potential scored across heterogeneous networks, the powerhouses located at the heart of the new economy had to rely on technologically enhanced infrastructures that would enable them to act as knowledge traps and innovation hotspots, while negotiating the tensions between local and global interfaces of exchange (Bathelt et al., 2004).

Subsequently, with the advances in understanding the social and cultural dimensions of innovation, the role of spaces and places as enabling factors in the competition for value creation has gained much analytical purchase thereafter. From cities and regions highlighted as the frontrunners of a rampant global economy (Asheim et al., 2007; Florida, 2009), to state-of-art research facilities (Gieryn, 2008; Lenoir and Alt, 2002) and corporate spaces (Allen and Henn, 2006; Garud et al., 2011), "contexts needed to be actively designed as an extension of intelligence" (Thrift, 2006a:292). Through its prime focus on novelty and emergent value regimes, this expanding corpus of enquiry has provided insightful accounts on the dynamics of innovation processes, but at the same time it also acted as a catalyst in mainstreaming elitist narratives and normative assumptions on what being innovative stands for. This has in turn fuelled some wideranging debates, for instance, on the 'over-romanticized' character of creativity (Jeanes, 2006:519) or the euphoria of success stories entertained by dominant innovation imaginaries, found to be flagrantly competing with the claim "that every person and every place can be a creative winner" (Peck, 2005:767).

In a more recent course of action, there is a growing commitment to approaches inspired by the creative potential that lies beyond the immediate reach of the 
performative economy. By and large, this timely spate of contributions seems to pivot on counter narratives articulated by micro-societal shifts and informal practices that persistently dog the logic of incumbent innovation imaginaries. Usually evoked under the heading of alternatives, they expose an astounding variety of more-or-less radical spaces of innovation, ranging from remote ice-fishing communities (Van Assche et al., 2013) and low impact developments (Pickerill and Maxey, 2009) to geographies of squatting (Vasudevan, 2015a), slums and favelas (Amin, 2014; McFarlane, 2012), all the way to the latest developments in experimental governance (Bulkeley and Castán Broto, 2013; Evans, 2011) and smart urbanism (Calzada and Cobo, 2015; Luque-Ayala and Marvin, 2015). Unveiled by this trend is an increasingly fluctuating landscape liable to shifting instances of core and periphery, which call into question notions of value creation, novelty or materiality. Accordingly, in her latest book, Anna Tsing (2015:65) eloquently captures the contingent character of alternatives in the discussion of 'salvage accumulation', showing how the fabric of capitalism is constantly reworked through the interplay between indigenous knowledge and dominant relations of production.

Most importantly, what many of these interventions arguably signal is the (still) poorly recognized fact that innovation processes are a common feature of any practice and hence, they afford a myriad of spatial, temporal and material inflections. Added to that, as Reviel Netz (2003:2) convincingly points out, "[s]hared beliefs are much less common than shared practices". This calls for a broader outlook on the emergence of alternatives, often to be recovered from the very dynamics of mainstream innovations, branching out beyond their original purpose. Moreover, the contingent character of mainstream and alternative innovations connotes processes of varying dynamics and rhythmic qualities, which appear to escape the sole grip of linear or cyclical interpretations. Instructed by this preliminary set of assumptions, the approach taken hereafter could be regarded as belonging to an amphibious domain of enquiry, operating along the interface between presumably grounded and more fluid readings of innovation processes. Aligned to the amphibious conceptual imaginary, there is also the thematic repertoire and empirical ambit of case studies explored within this dissertation. As such, the evoked conceptual liminality dictated the particular focus on amphibious practices, as the referents of material and affective dispositions, as well as of narratives of belonging scored across land-water interfaces.

The main case studies presented in chapters IV and $V$ were the result of an exploratory phase, with its point of departure in a pilot study conducted on the emergence of floating urbanization solutions in the Netherlands. The surveyed modalities of inhabiting land-water interfaces led me to wonder on the existence of alternative conditions of possibility to what otherwise appeared and were also tagged as very innovative attempts to reimagine urban dwelling. This struck me as a thorny task: 
where do you start in qualifying something as innovative or not? It took another survey of historical practices and some lengthy reflection sessions to realize that beyond the shifts and turns it has supposedly informed, innovation is much more performative than I initially thought. Thus, I started conducting ethnographic fieldwork by focusing on a pretty unusual case - floating churches, in Volgograd, Russia, more rural than urban, and definitely not the kind of instance you would run across in the mainstream innovation literature. The second case selection followed more or less the same oddly-informed pattern, this time - an on-land harbour, the brainchild of an experimental self-sufficient community recently established in Amsterdam, The Netherlands.

Given the above circumstances, I would like to sound a note of caution from the outset that grappling with these cases and the treatment of innovation will involve a rather convoluted journey, more experimental than canonical, yet hopefully lucrative enough in yielding a fresh perspective on the question of alternatives.

\section{I.1. Objective and research questions}

In studying innovation, perhaps the most immediate and striking aspect to consider is the unprecedented proliferation of political agendas it inspired over the past decade or so. The euphoric flagging of domains, practices and charismatic figures that previously manifested little to no reflexivity on their innovative condition arguably substantiates the performativity, as well as the liberating potential of the present state of affairs. Speaking from the field of Cultural Geography such an endeavor appears to be an opportune exercise, particularly for better understanding the underlying conditions of the current innovation ethos and the ways it (potentially) shapes future trajectories. Thus, the main objective that drives this dissertation is to investigate the emergence of alternatives in relation to dominant innovation imaginaries, through the spatiotemporal and material conditions they inform. Further on, the investigation draws on three main research questions, which address the meanings (1), workings (2) and expectations (3) connected to various innovation imaginaries, as follows:

(1) In what ways do different amphibious practices acknowledge the spatiotemporal and material conditions of innovation?

(2) How do those conditions enable the emergence of alternative innovations?

(3) To what extent are emergent alternatives influencing incumbent political repertoires as part of the current innovation ethos?

In answering these research questions, the dissertation brings into dialogue multiple disciplinary filiations and, as a secondary and more subtle objective, it reflects upon a new set of spatial (and temporal) imaginaries that would add up to the emergent 
spatial grammars currently animating geographical thought. Within the broader ambit of unpacking the workings of innovation processes, the theoretical and empirical exploration weaves contributions to the burgeoning strands of work on topological thinking, geographies of religion and secularism, archival practices and knowledge mobilities, urban progressive movements, and particularly, to the ongoing debates on 'new materialism' (Steinberg and Peters, 2015). As such, the methodological sway of this study covers a spectrum ranging from grand theory to ethnographic accounts of microsocietal shifts.

\section{I.2. Methodological considerations}

To negotiate the discrepancies of the above modalities of enquiry, the methodological approach taken in this project relies on a series of genealogical exercises that address the basics of normative distinctions, such as the interplay between innovation and imitation. The resulting observation repertoires combine the use of more conventional toolkits with experimental associations, which would bring us closer to what Deleuze and Guattari (1987:145, emphasis added) describe as diagrammatic reasoning: "taking regimes of signs or forms of expression and extracting from them particles-signs that are no longer [or not yet] formalized but instead constitute unformed traits capable of combining with one another." This strategy serves in unveiling new adjacencies, where being adjacent "may or may not imply contact but always implies absence of anything of the same kind in between" (Rabinow, 2008:33). Along this line of reasoning, the methodology shares a commitment to processual and practicecentred approaches, which emphasise the performative and contingent character of relationality and orders. In simple terms, practices ‘craft realities' (Law, 2004:113).

Subsequently, practices should be understood through their historical and spatial specificities, as "dialogical and processual", as mobile rather than static, and "responsive", meaning that they often "entail unpredictable, or unintentional, outcomes" (Cadman, 2009:4). In the study of innovation, these idiosyncrasies and volatile protocols are paramount to unpacking the functional domains of, and synergies between, various practices. De Certeau (1984:43) presses this point, by suggesting that practices "depend on a vast ensemble which is difficult to delimit but which we may provisionally designate as an ensemble of procedures", with the latter representing "schemas of operations and of technical manipulations". While spatiotemporal and material conditions are identified as key features in the treatment of innovation, it should be noted that the investigation pivoted primarily on the role of relations.

More specifically, particular attention was dedicated to the couplings that inform emergent alternatives and their functional domains. Here diagrammatic reasoning was 
at its peak in tracing how sometimes unconventional synergies - across heterogeneous functional domains - lead to processes of accumulation and shifting value regimes, which spark off alternatives to dominant innovation imaginaries. Therefore, any of the innovations under scrutiny plays second fiddle to "its relation to the social aggregates of which it forms a part" (Lorenc, 2012:80). Addressing the often volatile character of these relations requires a more generous reading of 'expressive action' (Thrift, 2008:118), to consider also the transient synergies between different practices that enable the "contingency of orders to morph into an explicit concern with the new, and with the chances of invention and creativity" (Anderson and Harrison, 2010:19).

In making a plea for the symmetry principle in scientific research, John Law offers the notion of method assemblage as "a way of thinking about all methods in the same terms, whether or not these fit normative rules about social science method" (2004:164). This is regarded as "the crafting or bundling of relations in three parts: (a) whatever is in-here or present (for instance a representation or an object); (b) whatever is absent but also manifest (that is, it can be seen, is described, is manifestly relevant to presence); and (c) whatever is absent but is Other because, while necessary to presence, it is also hidden, repressed or uninteresting" (Law, 2004:161). To follow up on Law, working through a methodological spectrum articulated by symmetry enables also a more reflective stance toward the research practice itself and its performative nature. And this has a critical impact on interrogating instances of inclusion and exclusion, ways of knowing and being, as well as the realities enacted through the political agendas of examined practices.

When taking the principles of symmetry and associativity seriously, Whatmore (2003:93) contends that ethnographic work comes "closest to the notion of 'generating materials', as opposed to 'collecting data', of any method in the social sciences", and this is redolent with the translation processes occasioned by the encounters between different practices (Latour, 1999; Mol, 2002). Accordingly, the ethnographic exploration underpinning this research has built upon three main methodological articulations. The first pertains to what Dwyer and Davies (2010) call animating the archives. This involves a broader outlook in the engagement with historical materials, based on an understanding of archives as mobile, and as subject to unremitting processes of negotiation. As they point out, such "work engages directly with the contradictory processes of archiving, of giving form to the identities and capacities of past communities" in "seeking to retain a dialogue between what can be made a lively presence and what remains a telling absence, whether in the built environment, urban or rural landscape", or as part of attempts "to document challenging labile environments of water and air" (Dwyer and Davies, 2010:89). 
The second methodological articulation was specifically concerned with the issue of performativity and the shifting roles of the researcher. On this point, I would like to emphasize the interplay between participant observation and observant participation techniques, with the latter implying "to understand and theorize the place of proactive, not just reactive participation”(Whitehead, 2009:5). The interplay involves here the attunement of methods to the vibe of different performances, from the unfolding of everyday life routines, to rituals and ceremonies or any unforeseen events. Some of the recursive techniques applied in these instances ranged from keeping a diary, recording audio and video materials and taking photos to "performing, talking, witnessing, sensing and listening" (Morton, 2005:668), as well as involving other participants in an active manner in the process of generating materials. Another lucrative method falling under this heading relates to modalities of narrating the self and the landscape (Wylie, 2005) or the embodied mobilities occasioned by various journeys and forms of encounter (Cresswell, 2010; Scriven, 2014). This proved particularly relevant in attaining to the action repertoires of amphibious practices and the multiple displacements they afford across land-water interfaces.

The third methodological articulation targeted the discursive realms enacted by the examined practices and how they translate into the (re)ordering of various material and affective dispositions. Complemented by the methods discussed above, a collage of semi-structured interviews, informal talks and field notes enabled a more comprehensive account on emergent narratives of belonging and their recurrent "patterns of similarity and difference" (Law, 2004:110). This exercise involved the exploration of contrasting imaginaries, of how they interfere with, and shape each other, or even morph into new narratives of belonging and creative action. And this last aspect brings me back to the notion of method assemblage, as a modality to think through the spectrum of what (and how) is made present, absent or Othered, in the process. While, "it is the emphasis on presence that distinguishes method from any other form of assemblage" (Law, 2004:84), there are always productive latencies resulting along such explorations, which make the playgrounds for imagination and critique. And in this respect, allow me to be surprised by the things that for any reason I did not take into account, or could not be openly evoked hereafter.

\section{I.3. Outline of the dissertation}

The dissertation is structured into seven chapters and its red thread could be envisioned as describing a loop between chapters II and VI, accordingly entitled The Magic Mirror I and The Magic Mirror II. Hence, the next chapter provides a critical overview of grand innovation narratives and their diverse filiations across Western thought, to outline the conceptual imaginary that drives this investigation. The 
thematic focus of The Magic Mirror I concerns the normative distinction between innovation and imitation, which arguably deters an ampler understanding of innovation processes. Chapter III, The surface and the abyss, expands on this preliminary vision through an extensive genealogical exercise to suggest some alternative imaginaries for the treatment of spatiotemporal conditions and connecting these to the empirical exploration presented in chapter IV, The floating churches of Volgograd. The latter dwells on an innovative development inspired by forms of religious ritual and related creative manifestations that resulted into an intriguing response to processes of identity formation and place-making in the Volgograd oblast, Russia. The fifth chapter, $A$ barbour on land, presents the case of an experimental self-sufficient community recently established in Amsterdam Noord, which through a makeshift sociotechnical arrangement seeks to redefine practices around sustainable and inclusive city-making. In answering the main research questions, both case studies discussed in chapters IV and V challenge a whole inventory of normative assumptions including, but not limited to, the interplay between formal and informal domains, secular and religious practices, mobility and stillness, rudimentary and advanced technologies or the functional status and materiality of things. The sixth chapter, The Magic Mirror II, closes the loop by connecting the findings to the introductory discussion from The Magic Mirror I, and elaborating further upon a more generous imaginary to tackle the workings of innovations, as well as the emergence of related alternatives. Chapter VII answers the main research questions and packs a final reflection in the form of some tentative corollaries informed by the findings of this exploratory journey. 


\section{II.The Magic Mirror I: Dis-locating innovation*}

"Two tasks: to defend the new against the old and to link the old with the new."

(Nietzsche, 1979:109)
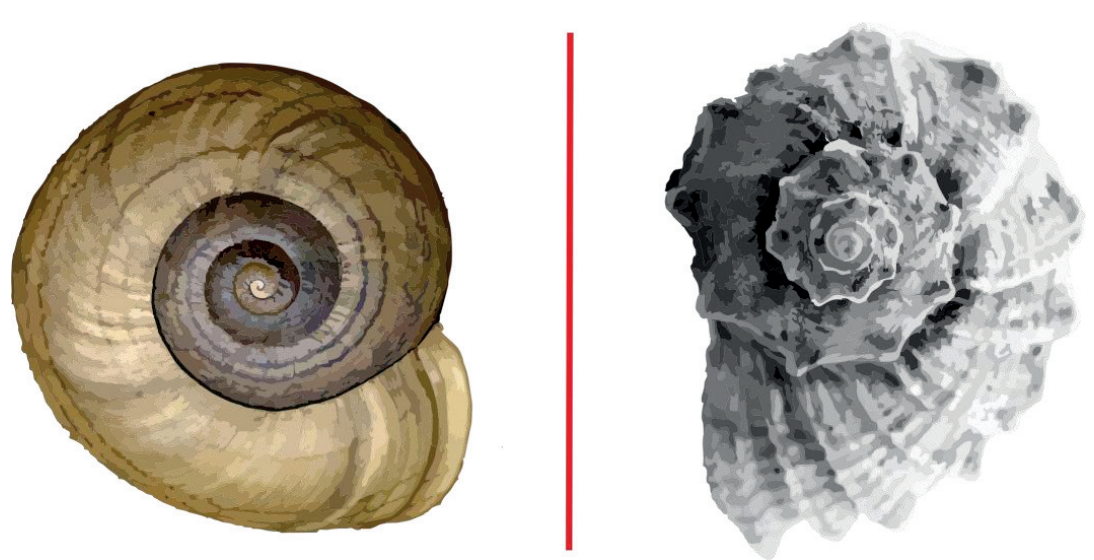

* This chapter includes excerpts from the essays published as:

Barba Lata I (2015) Topology and object formation. In: Beunen R, Van Assche K and Duineveld M (eds) Evolutionary Governance Theory: Theory and Applications. Cham: Springer, pp. 155 - 165.

Barba Lata I (2014) Review - Rob Shields' Spatial Questions: Cultural Topologies and Social Spatialisations. Society \& Space Open Site: https://societyandspace.com/2014/10/02/shields/ 


\section{The Magic Mirror I: Dis-locating innovation}

Any scientific enquiry regardless of its ambit or methodological layout rests upon a set of qualitative distinctions, and so does this dissertation. In light of the introductory notes, I commence by giving a spin to the concept of innovation and concurrently to its dislocation. As such, this chapter dwells on one of the fundamental questions in the treatment of innovation. This concerns the relation between innovation and imitation (Girard, 1990; Tarde, 1903). To unpack their interplay, and outline the conceptual imaginary that drives this investigation further, I take a critical overview of related grand narratives and their diverse filiations across Western thought. This could be regarded as a transgressive geographical exploration, one that seeks to reveal alternative conditions of possibility through the way it pieces together the spatial and temporal dimensions of innovation. As point of departure let us go through a brief mapping exercise, since it often happens to face a hitch when my interlocutors learn about the interest I have in innovation. Recurrent questions tap into the kind of innovations that fall within the scope of my work, including further distinctions along disciplinary filiations and, not least, what is actually being interrogated: the concept, the process or the product? So, where does it all the start? And, how can one tell what counts as innovative? The immediate answer is that anything goes, or even better, that anything matters. In avoiding to pick up a quarrel, the answer is usually complemented by a more or less lengthy story - as the one spanning the following chapters - and while audiences shift, the story shifts too in finding some familiar grounds to make sense of what is thrown in-between. And yet, as the story goes, beyond the scholarly gymnastics, ultimate resolutions are as unlikely as having ultimate innovation champions.

This preliminary instance of dislocation pertains to the discursive realms enacted by various appropriations of the concept (meanings), process (workings) and product (expectations). Far from trying to account on what appears to be a straightforward progression, the threefold split alludes to the intricate dynamics that informs the emergence of competing innovation imaginaries. Here, I refer in particular to the mounting interest in the study of innovation and the retake of grand narratives, as the one of creative destruction, which have grown over the past decade or so far beyond the more traditional fiefs of economics, organisation studies or political theory (Cowen, 2009; Topol, 2012). With more strands of work laying a claim over the meaning of innovation, the concept itself has become a floating signifier displaced through all sorts of referents into new combinations that add in making the picture even more 
complex. The current innovation ethos feeds onto that, to the extent that it has acquired an axiomatic of its own. Attaining to the dislocation work that takes place both within and in-between these discursive realms calls for a processual reading of innovation, one that is inevitably generous in breadth. This means that while some normative distinctions still hold in maintaining a fair degree of familiarity, many others will fade into a domain of loose connections and peculiar hybrids. In what follows, I address these questions in relation to the performative dimensions of innovation and the multiple inflections they afford across various strands of thought. The interplay between innovation and imitation is key to this endeavour, on one hand, to understand the evolution of particular innovation narratives and, on the other, to identify more lucrative modalities to interrogate the emergence of alternatives.

\section{II.1. Performative dimensions of innovation: the road to creative destruction}

"It should be known that war is universal, that strife is justice, and all things come into existence by strife and necessity." (Heraclitus, cited in Thom, 1989:55)

In echoing much of the work on innovation, a common denominator appears to be the high premium placed on creativity, competition and, above all, success. Consequently, as soon as the question of value enters the picture, success usually translates into success at the expense of others. As we will see later on, this perspective is neither novel nor a capitalist invention as often asserted, though it served the latter as an indispensable companion since its inception. More specifically along this cut, value is intimately connected to the commodification of innovations, i.e. to their applications and the returns they generate. Drawing on the distinction between invention and innovation, Schrader argues that ultimately "innovation is invention and exploitation" (cited in Allen and Henn, 2006:8). Schrader's fairly basic definition proves nonetheless salient in capturing the dynamics of innovation processes in what concerns their creative and exploitative dimensions. In a similar vein, Akrich et al. (2002a:205) regard innovation as "the art of interesting an increasing number of allies who will make you stronger and stronger".

Dating back at least to Adam Smith (Kurz, 2016), the catalytic effect of competition for new value creation has a long record as a core theme in economics. This is the arena where the creative destruction argument earned its reputation and has become fashionable again in light of the latest twists of the global economy. Joseph Schumpeter is generally credited with having exposed the destructive aura of innovation, as well as with having formalised its modern use in economic theory (McCraw, 2007). From his early works to the more recent insights of evolutionary economics, this morality of innovation seemed tailor-made for explaining the "cyclical nature of the capitalist economy" (Perez, 1983:359). In Capitalism, Socialism, and 
Democracy, Schumpeter (1975:83) contends that the "process of Creative Destruction is the essential fact about capitalism", as "what capitalism consists in and what every capitalist concern has got to live in".

Formalising innovation - and its creative destructive sway - as the main vehicle of capitalist (re)production distinguished as one of the most remarkable moves in the modern history of economics. To account on the evolutionary character of capitalism, Schumpeter (1975:85) resorts to the contextual analysis of particular innovations, i.e. the ways conditions for success are enabled at a given time within "the perennial gale of creative destruction". However, while his argument invites to retroactive inspection in order to tackle the impact of innovation processes, it also reinforces the inescapable and somewhat paradoxical logic of the creative destruction overtone. Taking a historical perspective on the matter, René Girard (1990:14) notes that "a truly innovative process, it is often so continuous with imitation that its presence can be discovered only after the fact, through a process of abstraction". In Anti-Oedipus, Deleuze and Guattari (2000:250) dwell at length on the reproductive logic of capital to signal that the "strength of capitalism indeed resides in the fact that its axiomatic is never saturated, that it is always capable of adding a new axiom to the previous ones".

Mirroring this reproductive logic, innovation processes are understood as coemergent with their environments, thus Akrich et al. (2002b:214) argue that in innovation research "[w]e must be ready at all times to burn that which we used to worship". As such, to bridge the chasm of post-factum interrogations and the emergent realities of an increasingly performative innovation ethos seems a thorny task. In other words, how can one in retrospect gain insight into something that is otherwise future oriented? Or, as Marilyn Strathern (1992:61) suggests, how to think innovatively about the future in making sure this is not 'trapped' by its present axiomatic? This obvious conundrum calls for a further examination of the creative and exploitative dimensions of innovation. To do so, the evolutionary perspective proves particularly accommodating at this point in unveiling the role of imitation or mimesis as politically Other to innovation processes, yet fundamental to their condition. Using Gabriel Tarde's (1903:3) account that "[s]ocially, everything is either invention or imitation" as a reference point, let us dwell briefly on the grand narrative of creative destruction.

Despite the late appropriation of the creative destruction motif in his writings, Schumpeter's figure is commonly associated with it, and for good reasons. The ingenious deployment of this simple but powerful metaphor to describe the workings of modern capitalism proved a veritable innovation in its own right. Implanted at the heart of economic analysis, the creative destruction mantra has become an enthralling narrative for both advocates and critics of capitalist modes of production (Ruttan, 
1959). And still, the roles of imitation and repetition were key not only to the reception or circulation of Schumpeter's conceptual enterprise, but also to its making. Deeply rooted in the history of human thought and belief, the idea of creative destruction appears throughout Egyptian and Greek mythology to Hinduism, with the latter having served as a rich source of inspiration for German philosophy during the late eighteenth and most of the nineteenth century (Reinert and Reinert, 2006). Along this genealogical filiation, Gottfried Herder's work on Vedic philosophy was seminal to other influential thinkers of the time, including Goethe, Schopenhauer and especially Nietzsche, who's treatment of creative destruction revealed a "new morality of innovation" that made its way from philosophy to the core of economic debates (Reinert and Reinert, 2006:56).

First used at the turn of the twentieth century in Werner Sombart's writings on modern capitalism, the creative destruction argument moved into the spotlight of German economics (Reinert, 2002). Schumpeter was no stranger to these ideas. As a fresh graduate from the University of Vienna, Schumpeter enrolled for political economy studies in Berlin, where he was exposed to the latest debates within the German Historical School and became "acquainted with Gustav von Schmoller, Werner Sombart, and other luminaries" (McCraw, 2007:57). Some commentators argue that while his perspective on innovation was praised in Anglo-Saxon economics for its originality, Schumpeter appears to have paid little recognition to the intellectual tradition he built upon (Chaloupek, 1995; Michaelides et al., 2010; Reinert, 2002). Thus, the weaving of convoluted genealogies of thought in Schumpeter's creative destruction imaginary would come closer to an instance of creative reuse, rather than a radical shift. Nonetheless, creative reuse distinguishes as a more inventive strategy than it might seem at first sight, and in light of the mimesis argument it holds the promise to reshuffle the historicity of innovation narratives.

If strife and necessity are the source of morphogenesis and concurrently of all 'inventions', a view that René Thom (1989:323) traces back to pre-Socratic philosophers Heraclitus and Anaximander, then mimesis scores as an evolutionary vehicle of utmost importance. Thom (1989) explains the branching of evolutionary trajectories through the notion of catastrophes, as generative events that trigger the displacement of organising centres and with them the replication of emergent properties. Along this line of reasoning, in Poetics of Space, Gaston Bachelard (1994:106) dwells on the vortical replication of matter, to suggest that animated by a transcendental geometry "life begins less by reaching upward, than by turning upon itself'. The primeval vortex that Bachelard evokes through the geometry of spirals has been a longstanding source of inspiration in the study of phenomena, from Archimedes and Lucretius to Descartes and Leibniz, and further on to Maxwell and J.J. Thompson (Serres and Latour, 1995; Thompson, 1945; see also, Châtelet, 2000; 
Deleuze, 2006). Tarde (2012:45) builds upon the latter's observations in pointing out that:

"Diversity, and not unity, is at the heart of things (...) Everywhere an exuberant richness of unheard-of variations and modulations springs forth from these permanent themes which are called living species and stellar systems, and from equilibria of all kinds, and in the end destroys and renews them utterly (...) Forces, we are told, exist to serve laws, and all laws apply to phenomena to the extent that the latter are perfect repetitions and not repetitions with variations; all laws manifestly tend to ensure the exact reproduction of the themes and the indefinitely prolonged stability of all kinds of equilibria, and to prevent their alteration or renewal."

Tarde's analysis of phenomena dogged by the laws of observation eloquently captures the evolutionary trajectories of innovation narratives as displaced by all sorts of political, scientific and, not least, religious dispositions. While digesting innovation in its astounding diversity is presumably a contemporary habit, the critical engagement with its symptoms appears as a less recent enterprise. From the Greek and Roman Antiquity, the earliest accounts on innovation (or what could be considered its referents) latently embed the tension of variation through mimesis as a drive for renewal and change. Analogous to these initial appropriations would be the distinction Romans drew between the Greek term symposium and convivium in what concerned customary dinner parties. As Mary-Ann Ray (1997:44) tells us, the former - which meant 'drinking together' - was often disallowed by "[s]ome revolutionary Romans, who preferred intelligent conversation to decadent entertainment".

Genealogical interrogations of early references to innovation insist on two main sources considered to have informed the various meanings attached to it thereafter. The oldest one appears to be the Greek kainotomeo, seconded by the Latin innovo (Godin and Lucier, 2014). The Greek historian and philosopher Xenophon used kainotomeo, with the meaning of 'cutting anew', to describe the opening of new silver mines (Godin and Lucier, 2012:8). Besides Xenophon's literal use of the term, later interpretations are found in the works of Aristotle, Plato or Polybius, where innovation is related to cultural and political change, yet bears mostly adverse connotations as "subversive and revolutionary" (Godin and Lucier, 2012:24). With the Romans, the use of innovo pertains first of all to spiritual renewal, informed by the adoption of Christianity as public religion in the fourth century (Godin and Lucier, 2014:13). The Latin import was particularly enduring throughout the Middle Ages. Still, the Reformation sparked another variation on the idea of renewal as redolent with original Christianity, advocating for "the authentic imitation of Christ, uncorrupted by Catholic innovation” (Girard, 1990:8). 
Drawing a parallel between Renaissance and Reformation, Tarde (1903:363, emphasis added) suggests that "each movement was a complete evolution in itself", with the former having proved for "a narrow group of souls reared in the aristocracy of art and intellect, a profound dechristianisation which, underneath the Reformation, was to spread among us in the eighteenth century". Animated by the progress of science and technology, the dawn of modernity was already heralding a fracture with the transcendental models of the past. The longstanding onus inherited from religious dogma, politics and even classical aesthetics became one of the strongest arguments to rehabilitate the idea of novelty (Eco, 2005). As such, the late eighteenth century is considered the turning point, which cultivated a renewed set of dispositions toward innovation. Emancipated from its past legacies, the impetus to innovate - this time in its subversive and revolutionary character - moved centre-stage in preluding the social reforms of the nineteenth century. The profound consequences of this shift are identified by some commentators with a reversal of the roles of innovation and imitation. According to Girard (1990:11, emphasis added), “[o]ur world has always believed that to be innovative and to be imitative are two incompatible attitudes. This was already true when innovation was feared; now that it is desired, it is more true than ever".

\section{II.2. The Magic Mirror I}

"It is possible to discover regularity in a limited domain of phenomena independently of other moments and other phenomena, which therefore can remain completely concealed from the intellectual observation (...) To maintain the certainty of observed regularity as long as possible, one tries to isolate systems, i.e., to exclude observations which disturb this regularity." (Brouwer, cited in Van Stigt, 1979:395, emphasis added)

Walter Benjamin's unfinished masterpiece The Arcades Project offers a remarkable account on the nineteenth century Parisian ethos - the inception of the modern metropolis under the turmoil of Haussmann's renovation programme and the rampant pace of new technologies. Cast against this background, his core concern with the commodification of things prefigured the emergent temporality of creative destruction: "[b]eing past, being no more, is passionately at work in things" (Benjamin, 2002:833). Taking fashion as the referent of accelerating instances of disposal or dissolution, for Benjamin capitalism acted as sort of "vast machinery for the production of just such remainders, objects that assume the status of enigmatic signifiers" (Santner, 2006:79, emphasis added). The detritus of commodity production and consumption opened in Benjamin's historical intuition alternative modalities to engage with the recent past and "achieve the not-yet-possible; one side of a dialectic which also sees the development of new technologies reciprocally offer possibilities for radically new forms of perception and cognition" (Calderbank, 2003:6, emphasis in 
original). This line of reasoning arguably strikes an undertone of fundamental relevance to the treatment of innovation in general, and of its exploitative dimensions in particular.

Whereas the idea of exploitation has proved particularly enduring in the guises of creative destruction and 'diffusionism' (McCann, 2011), the more subtle character of variation through mimesis and even creative reuse deserve further attention in exposing alternative ways to digest innovation. Echoing Girard's (1990) point on the seemingly irreconcilable tensions between imitation and innovation, whether or not considering the reversal of their roles as a feature of modernity, they yet emerge as inherently woven through the question of accumulation. In fact, when taking this forward in terms of value creation, the residual substrate which underpins 'successful' innovations distinguishes as particularly important. As Benjamin (2002:788) notes, "success breeds imitation, and it is rare that the imitations do not bring out the troublesome aspects of the things they copy". Driven by a broad range of dispositions, these instances of appropriation through mimesis, trial and error, are most often scored across the problematic latencies of things and, as such, they can inspire alternative innovations. Besides, once the contingency of the imaginaries they inform is acknowledged, the picture becomes much clearer. Tarde's (1903:366-367, emphasis added) profound reflection on this matter has it that:

"The supreme law of imitation seems to be its tendency towards indefinite progression. This immanent and immense kind of ambition is the soul of the universe. It expresses itself, physically, in the conquest of space by light, vitally, in the claim of even the humblest species to cover the entire globe with its kind. It seems to impel every discovery or innovation, however futile, including the most insignificant individual innovations, to scatter itself through the whole of the indefinitely broadened social field. But unless this tendency be backed up by the coming together of inventions which are logically and teleologically auxiliary, or by the help of the prestige which belongs to alleged superiorities, it is checked by the different obstacles which it has successively to overcome or to turn aside. These obstacles are the logical and teleological contradictions which are opposed to it by other inventions, or the barriers which have been raised up by a thousand causes..."

Tarde's ecological perspective on innovations, as co-emergent with their environments, exposes an intricate meshwork of evolutionary trajectories that are subject to varying intensities, rhythms and momenta. Approached in this non-linear fashion, the obstacles that Tarde invokes to account on competing inventions afford a more dynamical reading, as crucibles to which "symmetry is always a moving target" (Law, 2004:173, emphasis added). With the branching of innovation processes, the resulting interstitial spaces become vibrant realms of loose connections and hybridity. In other words, they pack the debris of creative destruction. Here, the role of variation through mimesis is at its peak, and accumulation thrives on what has been consumed or Othered 
via dominant processes of accumulation and value creation. Caught in-between worlds that are similar yet different, these are the breeding grounds for bybrids gone mobile along asymptotic trajectories that often reanimate the sediments of seemingly established orders. In Deleuze's (1994) terms, the resulting evolutionary trajectories pertain to the problematic domain, where the proliferation of multiplicities constantly dogs axiomatic distinctions. Through a vortical logic of fine adjustments and minor deviations, mimesis becomes in this context a "generative differential element" (Deleuze and Guattari, 1987:489, emphasis added). As such, the norm of this myriad minor successes is tinkering, and their glory lies in creatively scavenging the residual substrate of what Tarde (1903:367) calls alleged superiorities. This is an issue of temporality, as much as one of observation, yet it is hard to fail noticing how life, materiality and affects endure despite the countless vicissitudes encountered along the way. Accordingly, Deleuze (1994:21, emphasis added) notes how:

"A bare, material repetition (repetition of the Same) appears only in the sense that another repetition is disguised within it, constituting it and constituting itself in disguising itself. Even in nature, isochronic rotations are only the outward appearance of a more profound movement, the revolving cycles are only abstractions: placed together, they reveal evolutionary cycles or spirals whose principle is a variable curve, and the trajectory of which has two dissymmetrical aspects, as though it had a right and a left. It is always in this gap, which should not be confused with the negative, that creatures weave their repetition and receive at the same time the gift of living and dying."

Thus, a more generous reading of variation through mimesis beyond the linear entrapments of historical and functional redundancy holds the promise of turning the creative destruction imaginary on its head, by unveiling its political Other, creative reuse. The latter would saliently enhance post-factum accounts on how evolutionary paths contract and expand, solidify and flow again, in fostering particular innovations at particular times. Hence, taking the creative reuse thesis seriously would unlock a domain of coiling progressions and (re)turns (a question explored at length in the upcoming chapters), very much in line with Benjamin's (2002:883, emphasis in original) treatment of the 'Copernican revolution in historical perception':

"Formerly it was thought that a fixed point had been found in 'what has been' and one saw the present engaged in tentatively concentrating the forces of knowledge on this ground. Now this relation is to be overturned, and what has been is to acquire its dialectical fixation trough the synthesis which awakening achieves with the opposing dream images. Politics attains primacy over history. Indeed, historical 'facts' become something that just now happened to us, just now struck us: to establish them is the affair of memory (...) There is a not-yet-conscious knowledge of what has been: its advancement has the structure of awakening." 
This is a move that even Schumpeter might have intuited in attempting to reconcile the two main canons of thought in economics (Reinert, 2002), which could also explain the later use of creative destruction in his work (McCraw, 2007). As indicated in the former section, tracing the displacements that occur along evolutionary trajectories is contingent on the laws of observation and the normative distinctions they inform. Calling these into question, Tarde (1903:46-47, emphasis added) contends that "[f]orms are only brakes and laws are only dykes erected in vain against the overflowing of revolutionary differences and civil dissensions, in which the laws and forms of tomorrow secretly take shape, and which, in spite of the yokes upon yokes they bear, in spite of chemical and vital discipline, in spite of reason, in spite of celestial mechanics, will one distant day, like the people of a nation, sweep away all barriers and from their very wreckage construct the instrument of a still higher diversity". On a similar note, George Gamow suggests that Einstein "was probably the first to realize the important fact that the basic notions and laws of nature, however well established, were valid only within the limits of observation and did not necessarily hold beyond them" (cited in Shields, 2013:147, emphasis added).

In mirroring the axiomatic of creative destruction through its material and affective Others, creative reuse challenges the very laws of observation that perpetuate an ethos of violence in a race, which can never be won. Its disguise is that of excess, and rests on cumulative processes that prize the residual surplus of things beyond the immediate affordances of disposal or dissolution. Seeping through the interstices of dissonant domains, creative reuse acts as catalyst for makeshift arrangements that habitually defy normative assumptions on what counts as innovative and what not. This in-betweenness bears the load of amorphous constellations of bits and pieces open to all sorts of synergies. Michel Serres coins these "spaces of interference", the realms of metaphor and metamorphosis that often carry "analogies, which are dangerous and even forbidden", yet essential, as the only "route to invention" (Serres and Latour, 1995:64-66, emphasis in original).

\section{II.3. Metaphor and metamorphosis}
"We might need to live in many worlds at once, to be many monads at once (...) Often we need to move in several directions at once, but we especially need to follow those trajectories that allow us to enrich our situation by transferring into it new architectures." (Plotnitsky, 2012:367-368, emphasis added)

In attempting to find a clearer expression to the meandering course of argument on metaphor and metamorphosis, M.C. Escher's lithograph the Magic Mirror (figure 1) proved an inspiring companion. The artwork brilliantly captures the interplay between symmetry and dissymmetry organised around the central motif of the magic mirror. 
This gives the impression of a permeable membrane, both transparent and reflective, which spawns two opposed processions of hybrid creatures (griffins). Each procession swirls around a sphere, to converge into a tessellated pattern from which they are extruded again through the mirror's surface. Resonating in multiple ways with the points raised thus far on the emergence of innovations (see for example the questions of bybridity, Othering or observation), the magic mirror provides also a potent metaphor to address the contingent character of creative destruction and creative reuse. In his genealogy of modern science, Gilles Châtelet (2000:182, emphasis in original) finds that the "bold metaphor forces the analogy and steps over degrees of proof (...) [i]t transports thought experiments, allusions from the old theory into the new: the latter gains a whole set of habits, the former a new rigour".

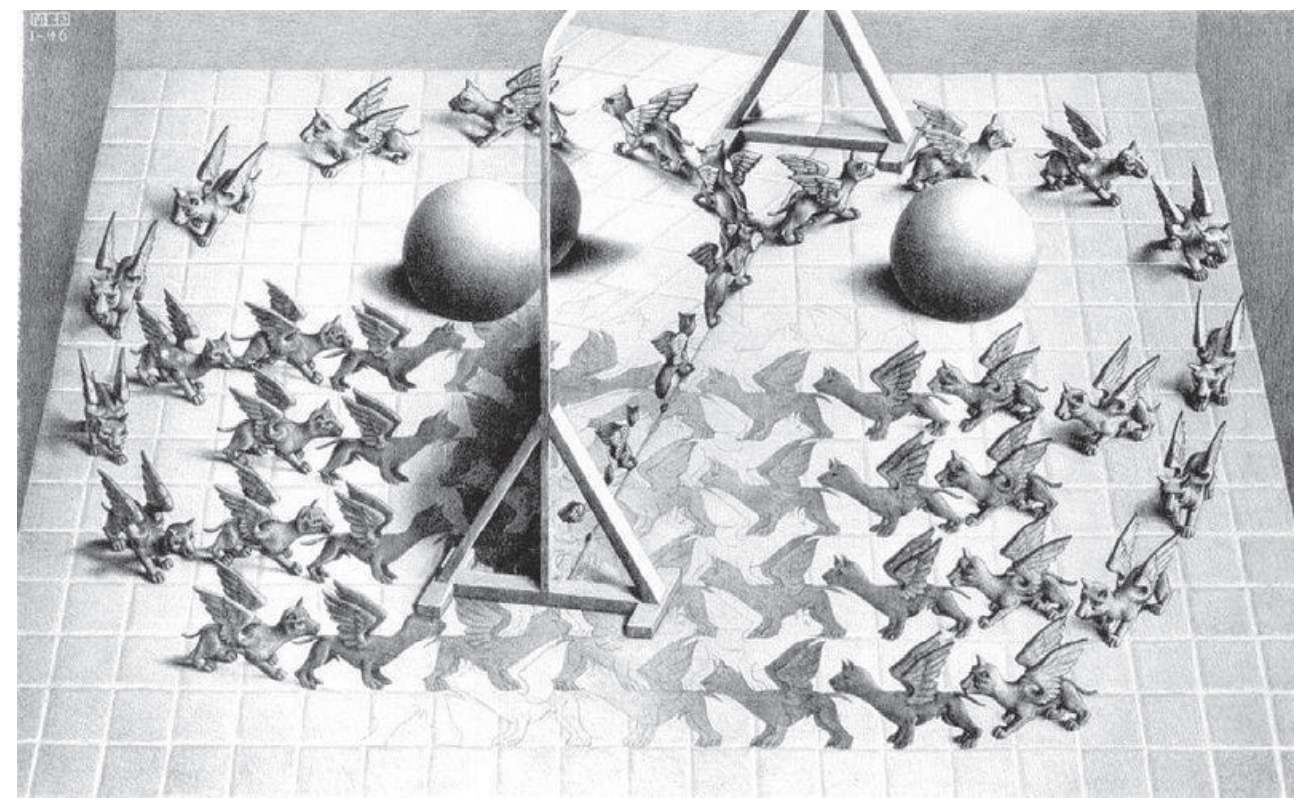

Figure 1 - Magic Mirror (1946), lithograph print by Dutch artist M. C. Escher. Source: All M.C. Escher works (C) 2016 The M.C. Escher Company - the Netherlands. All rights reserved. Used by permission. www.mcescher.com

This treatment of metaphors encapsulates the innovative potential of what Tarde (1903:207, emphasis in original) calls "progress from within to without", a perspective that rests upon two main axioms, namely: "[t]hat imitation of ideas precedes the imitation of their expression" and "[t] hat imitation of ends precedes imitation of means". To draw a parallel with the magic mirror lithograph, the branching trajectories that mimic ideas and ends inform the proliferation of expressions and means. In tune with Tarde's reading, this logic of differentiation distinguishes as an enabling factor for alternative conditions of possibility and creative action. Consequently, the 
meanings, workings and expectations related to these alternative innovations are dislocated along the seams of worlds fusing with each other. The liminal spaces generated as such become the transient cradles of unusual synergies and forms of attunement that recurrently usurp previously held assumptions on the status of things and affects (see chapters IV and V). Attaining to these spatiotemporal and functional displacements would bring us closer to Deleuze's (1994:162) topological treatment of the problematic, as an expression of multiplicities and which "tends to give rise to discontinuity on the basis of continuity, or to ground solutions in the conditions of the problems".

Topology is commonly recognised as the branch of mathematics that studies geometrical objects and more generally spaces, which maintain their properties under continuous deformations. In contrast to geometry, topology examines the structure of spaces and their functional continuity, rather than their measurement or scale (Plotnitsky, 2006). Thus, in topology, seemingly different geometrical objects as the cube and the sphere - but this applies also to more abstract mathematical entities are considered to be equivalent if they can be continuously deformed from one into the other. This has to do with connectedness, a basic topological property that concerns the structure of topological spaces (their constitutive elements) and the relations (paths) holding them together (Totaro, 2010). As Alexandroff (1961:8-9, emphasis added) tells us, a "topological space is nothing other than a set of arbitrary elements (called 'points' of the space) in which a concept of continuity is defined. Now this concept of continuity is based on the existence of relations, which may be defined as local or neighborhood relations - it is precisely these relations which are preserved in a continuous mapping of one figure onto another (...) Moreover, this idea of a space depends only on these relations and not on the nature of the respective objects".

The genealogy of these ideas dates back to Leibniz's work on geometria situs and analysis situs, which describe a form of positional calculus developed for the study of geometrical figures based on their location in space (Durie, 2006). Leibniz's enterprise has inspired some of the most important contributions to the inception of topology in mathematics, among which, the works of Bernard Riemann or Henri Poincaré are legend. The ground-breaking achievement occasioned by topology was intimately connected to its conceptual treatment of space. Through this novel conceptualisation of space, which made possible synergies between different strands of thought in the discipline, topology played a fundamental role in reshuffling the very space of mathematics. According to Totaro (2010:395), the "line of thought introduced by pioneering topologists like Riemann is simple but powerful. Try to translate any problem, even a purely algebraic one, into geometric terms. Then ignore the details of the geometry and study the underlying shape or topology of the problem. Finally, go back to the original problem and see how much has been gained". Along this line of reasoning, one of the most important applications of topological thinking enabled the 
study of functions in relation to the spaces they articulate (Hilbert and Cohn-Vossen, 1990). The further interrogation of creative reuse pivots on this perspective, which informs the conceptual, as much as the empirical explorations scored across the following chapters. Accordingly, the next chapter entitled The surface and the abyss, dwells on a more in-depth account on how the inception of topology provides evidence for an ethos inspired by creative reuse, which keeps recasting all sorts of surprising returns. 



\section{The surface and the abyss/Rethinking topology*}

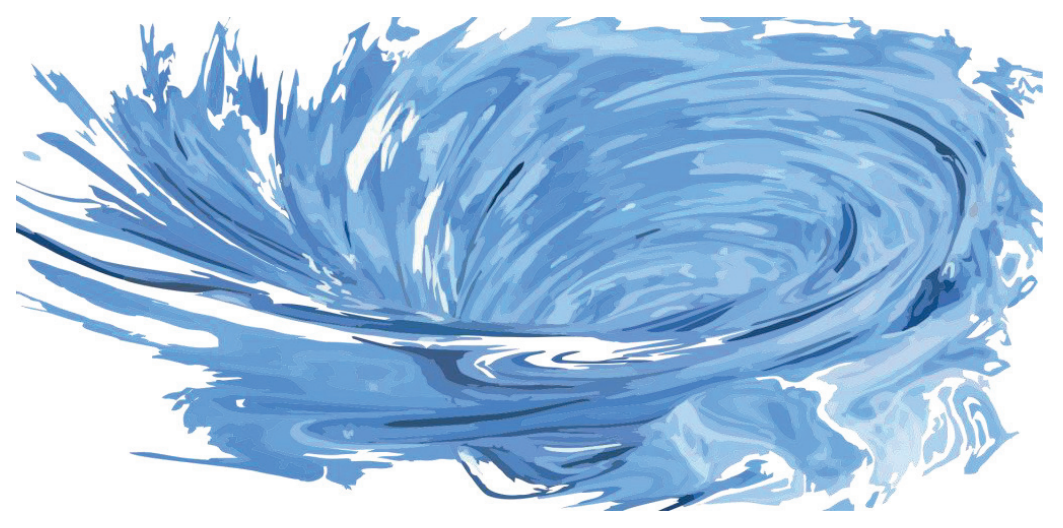

*This chapter is published as:

Barba Lata I and Minca C (2016) The surface and the abyss/Rethinking topology. Environment and Planning D: Society and Space 34(3): 438-455. 


\title{
III. The surface and the abyss/Rethinking topology
}

\begin{abstract}
Through a critical deployment of the surface/depth metaphor, this article explores the catalytic potential of topological thinking to establish points of articulation between apparently opposed notions and canons of thought. Starting from a genealogy of mathematical developments and philosophical mediations toward the end point of geography, we address the interplay between the formal (axiomatic) and conceptual (problematic) dimensions of topology in suggesting some potentially alternative ways of re-imagining the role of topological thinking for spatial theory and human geography.
\end{abstract}

\section{Keywords:}

Topology, surface/depth, mathematics, spatial theory, human geography

\begin{abstract}
"Deafen yourself to the noise of the expressible! Listen instead for the whisper of the taken-for-granted!” (Gunnar Olsson, 1982:224).

"The existing scientific concepts cover always only a very limited part of reality, and the other part that has not yet been understood is infinite. Whenever we proceed from the known into the unknown we may hope to understand, but we may have to learn at the same time a new meaning of the word 'understanding"'(Werner Heisenberg cited in Majid, 2012:98).
\end{abstract}

\section{III.1. Introduction}

Discussions invoking foundational issues are always a sensitive terrain. They often provoke tensions in the bundle of red threads underpinning the ontological status of disciplinary domains. Consequently, new ways of passage are sometimes exposed between familiar and less familiar places, often leading to the erosion of formalised categories and their assimilation into new architectures that challenge conceptual grounds once considered safe havens. This is also true for any plea for more inclusive approaches in current geographical conceptualizations of space, like the one emerged a few years ago with John Allen's call for a 'topological twist' (see, Allen, 2011; 
Coleman, 2011; Elden, 2011; Latham, 2011; Paasi, 2011a). Topology currently scores as one of the putative core topics in geography, but despite the proliferation of topological imaginaries of the past decade or so, it is not clear as yet whether what we are facing is the manifestation of a fashionable trend or something with more profound implications for spatial theory (for a tentative mapping of these see, among others, Martin and Secor, 2013). As such, we are still left with some important questions concerning for instance the issue of 'topological returns' (Paasi, 2011a; Phillips, 2013), or of how to make better sense of topology in analytical and methodological terms (Lorimer, 2007; Shields, 2013).

Arguably, there are many alternative ways to address these queries. Along the interventions that lament over the metaphorical treatment of topology (Shields, 2013) or the often superficial engagement with its 'genealogies of meaning' (Abrahamsson, 2012; Elden, 2011), we propose a genealogical exercise staged through a critical reflection on the surface/depth dichotomy, as a long-standing legacy in Western thought (Tuan, 1989). Echoing Heisenberg's quote above, our exploration may thus be conceived as a passage between the visible and the invisible, the known and the unknown, but also as an expression of the shifting spatialities articulated by appropriations of topology in geography. Working from a broad understanding of topology - which builds upon some key conceptual filiations to its establishment as a branch of mathematical endeavour - we address the interplay between its formal and problematic dimensions, emphasising the underrated potential of the latter for spatial thought.

Our main objective is thus to show how recourse to a tentative genealogy of topology in mathematics could unlock alternative venues for topological thinking in human geography and its approach to spatial theory. We attempt to do so first by reflecting on present-day treatments of topology in geography and by suggesting that a more direct reference to the mathematical tradition in topological thinking could prove useful for a broader geographical audience. The genealogical exercise developed in the second part of the article is therefore specifically preoccupied with how the unfolding of topological thinking has affected the conceptual space of mathematics, something that may arguably inspire alternative ways for geographers to adhere to the 'topological promise'. In the final part we return to the critical reflection on the interplay between the formal and conceptual dimensions of topology, to highlight the potential contribution we envision for topological metaphors and topological thinking in advancing conceptual work in geography. 


\section{III.2. The surface and the abyss}

In order to better understand how our engagement with topology fits contemporary epistemological concerns in geography we would like to start by clarifying the critical use of the surface/depth dichotomy as a tool for analysis. In Surface Phenomena and Aesthetic Experience, Yi-Fu Tuan (1989:240) argues that "cultural geographers-cumstorytellers stand only a little above their material and move only a little below the surfaces of reality in the hope of not losing sight of such surfaces". Taking up Tuan's point, we work through the discursive tensions underpinned by the classical dialectics of outside and inside, surface and depth, in an attempt to rethink the role of topology for spatial theory. Thus, we critically deploy the surface/depth metaphor as an overarching motif in discussing the pivotal relation between the formal and conceptual - or in Deleuzian (1987) terms, the axiomatic and the problematic - dimensions of topology, in both geography and mathematics. As a first step, we proceed from an overview of the geographical treatment of topology and some of its most important filiations.

From the advent of post-structuralism and the widely celebrated relational turn, the role of philosophy as a mediator - to speak with Serres (1995) - between 'the hard and the soft' has been paramount to the evolution of geographical readings of space. The works of Bachelard, Derrida, Deleuze and Guattari, Serres and, more recently, Badiou, hold a critical importance in this respect. To recall Badiou's (2006:22) argument on the indispensable condition of philosophy to engage with mathematics, "a condition that is at once descriptively external and prescriptively immanent", the same could perhaps be argued about the condition of geography concerning its intimacies with philosophy and, occasionally, mathematics. What has grown out of the different translations and mediations of these accounts seems to be a more profound understanding of how space and time bend under different forms of encounter that bring into being a fluid "universe of spaces" (Thrift, 2006:139), where nothing is fixed or bounded anymore. Along the strands of thought stimulated by the infusion of relationalism - of cultural, economic, more-than-human and more-thanrepresentational geographies - we have witnessed the development of a complex spatial lexicon endorsed by an unprecedented proliferation of new imaginaries and conceptual motifs.

On a more subtle level, this development arguably echoes Deleuze and Guattari's (1987) treatment of major and minor science or Châtelet's (2000) take on the history of ideas, which capture in an eloquent manner the dynamics of the axiomatic and the problematic in relation to spatial thought. For Deleuze, the problematic as key feature of minor science emerges as an expression of multiplicities and distinguishes through its topological qualities. As he points out in Difference and Repetition by resorting to Riemannian geometry - discussed in the following section - "[t]o solve a problem is 
always to give rise to discontinuities on the basis of a continuity which functions as Idea" (1994:162). In a similar vein, Châtelet (2000:69) dwells on the interplay between two rhythms that articulate the history of ideas, "one, completely discontinuous, of 'ruptures', 'paradigms' and their refutations, and the other of the problematic latencies that are always available for reactivation". These accounts parallel one of the enduring conundrums in the history of ideas and geography's main concern - that of space as master category and form of knowledge, as transcendental and immanent.

Despite being refracted through several strands of thought, the above perspective has frequently (re)surfaced in various debates across the social sciences and particularly geography. According to Thrift (2008:120), "understanding space-times requires new 'geometric' metaphors that are able to describe them in their own - heterogeneous terms and can take full account of the number and nature of other actual and possible space-times". Following this line of reasoning, the resulting proliferation of imaginaries and conceptual motifs is evocative of the 'problematic of space' but also of the liberating potential some have found in topology, to either add more substrate to or even challenge the geographical conceptualisations of space. Attempts at rethinking the interplay between local and global - complemented by debates around the elusive character of borders, scales, territories, regions or networks - have all taken their share of the topological over the past decade or so (Allen and Cochrane, 2010; Amin, 2007; Latham, 2002; Macleod and Jones, 2007; Mezzadra and Neilson, 2012; Thrift, 2008; Urry, 2005). As such, the growing adherence to the topological promise has reflected in an ever-expanding set of concerns falling under its sway, including sequels to some of the enduring dialogues in geography over the relation of state/territory to biopolitics and governmentality (Collier 2009; Giaccaria and Minca, 2011; Hannah, 2006). The effects of this are manifest through the sheer variety of topological repertoires articulated by both theoretical and empirical concerns and as "[c]ommonly non-territorial, non-linear and non-cartographic, the search for a topology to satisfy seldom seems to settle for very long” (Lorimer, 2007:94).

Part of the current trend, initial deployments of topology in geography have largely built upon the works of Serres, Deleuze and Guattari, and especially by drawing inspiration from actor-network theory (ANT) and Science and Technology Studies (STS) accounts (Murdoch, 2006). The interlocking of the established relational perspectives in geography (Harvey 1997; Massey 2005; Thrift 1996) with those of ANT and STS (Callon and Law, 2004; Latour, 1993; Mol and Law, 1994) has fostered a renewed interest in questions of materiality and agency, bringing along the promise of a spatial lexicon better fitted to account on the multiplicity of space-times resulting through their various intersections. This has proved anything but a smooth transition, in which emergent imaginaries coupled to a broad range of empirical concerns have sought to challenge both the status of established categories and the scope of 
geographical enquiries. In this regard, Annemarie Mol's and John Law's after-ANT revisionist agenda and its topological ethos (Vasantkumar, 2013) scores among the most prolific sources in the geographical appropriations of topology. Added to the infusion of 'elemental' spatialities - of fluid, fire, air and gel (Law and Mol, 2001; Serres and Latour, 1995; Sheller, 2004) - and their topological referents, the growing commitment to various 'affective materialisms' (Anderson and Wylie, 2009) and processual philosophies (Merriman, 2015) have given human geography a more acute sense of intellectual fragmentation, but also a certain flavour of post-disciplinary engagement (Lorimer, 2007).

In addressing the issue of object alterity, Law (2002:102) stresses that '[ $\mathrm{t}$ opology generates spaces by creating rules about what will count as homeomorphic objects and there is no limit to the possible rules and spaces'. Accordingly, the panoply of research objects appropriated in geography through the onus placed on exposing an astounding variety of more-than-human worlds, as well as on questions of performance, emotion and affect, has marked a significant shift by entertaining a "host of spatiotemporalities that previously might not have even been considered properly geographical" (Latham, 2011: 313). In line with the empirical foci and experimental conditions that topology has enabled for social and cultural theory, we find explorations of the psychological dimensions of space (Blum and Secor, 2011), tactility and contagion (Dixon and Jones, 2015), neurosurgical practices (Moreira, 2004), biomedical prevention technologies (Michael and Rosengarten, 2012), humanitarian spaces (Fredriksen, 2014), digital media and socio-technical change (Fuller and Goffey, 2012) or of performing arts and videography (Salazar Sutil, 2013). Far from being an exhaustive review, this note is merely suggestive of the fecundity of topological repertoires in geography and beyond. However, when considering the principle(s) of multiplication behind this expanding universe of spatial imaginaries, the treatment of topology seems to fall short where it could perhaps prove most lucrative, i.e. in challenging not only the axiomatisation of established geographical categories but also of the emergent ones .

After many disparate contributions and several special issues appeared in some key journals (Configurations, 2009, 17:1-2; Society and Space, 2012, 30:2; Theory, Culture \& Society, 2012, 29:4-5; Space and Culture, 2013, 16:2), often heralding a 'topological agenda' (Lury et al., 2012) in social and cultural studies, we are still somehow left with a vague sense of how topology might fit into the playground of geography. The resulting abundance of topological imaginaries and motifs arguably exposes the problematic dimension of topology, confirming - at least for the time being - its status as the ultimate engine of relationalism. Twisted, stretched and bent, the topological proves to be what Girard and Derrida referred to as the pharmakon, "a mimetic drug which is both remedy and poison" (Smyth, 1997:223). Resorting to it has already 
stimulated a growing commitment to approaches that question the arsenal of established categories in the discipline; however, this seems to have happened only to a limited extent, and rather intuitively. This issue is signaled by various critical interventions pleading for more inclusive approaches in the use of topology (Jones, 2009; Martin and Secor, 2013; Paasi, 2011b), whereas other commentators call into question its ontological underpinnings in relation to core geographical categories, as those of space and place (Malpas, 2012), or its metaphorical treatment (Shields, 2013). Yet, although supporting the imperative for more clarity, it does not mean that we are resisting the use of topological metaphors, quite the contrary. As the surface/depth metaphor shows, we are buying into this strategy but in a slightly different manner.

While acknowledging the explanatory power of tropes such as 'rubber-sheetgeometry' or of different analogies with topological figures and their properties, we are left with the impression that the predominant concern with the formal/axiomatic dimensions of topology (i.e. what the basic axioms of topology tell us) has inhibited the engagement with its 'problematic latencies' (Châtelet, 2000) and their potential to foster new points of articulation in the conceptual apparatus of geography. Thus, in this context we envision the critical role of the surface/depth metaphor in capturing the dynamics between the axiomatic and problematic of topology, to reveal alternative possibilities for creative action in spatial thought. The interplay between the two modalities lies at the core of Deleuze and Guattari's (1987:485-486) treatment of major and minor science, in which the former "has a perpetual need for the inspiration of the minor; but the minor would be nothing if it did not confront and conform to the highest scientific requirements". Along this line of reasoning, Abrahamsson (2012) suggests that the approaches that have alimented the debate over the topological 'twist' in geography have generally manifested a poor engagement with the original conceptual grounds where these ideas took shape. Accordingly, by dwelling on a critical reflection that considers the inextricable condition of the axiomatic-problematic, we think there is more creative potential at stake in the spectrum of topological thinking. However, in order to make this more explicit, some further clarification is perhaps of help.

Following up on Elden's (2011) call for historical specificity, we suggest that the treatment of topology should not be separated from its complex trajectories across different fields of knowledge. We are not advocating here to turn to mathematics or philosophy as higher authorities in what Massey (1999:264) describes as 'reverential reference'; however, we believe that discarding the genealogies that have articulated the spectrum of topological thinking may lead to a relatively superficial engagement with topology in geography debates. In Topology, Algebra, Diagrams Rotman (2012:255), for instance, draws on Poincaré's and Châtelet's ideas to explore the potential of diagrams through "the pivotal role they play in mathematical ontogenesis" by 
capturing gestures and the embodied dimensions of space. Providing a similar argument, Netz (2010) contextualizes the topological character of ancient mathematical diagrams through an insightful discussion of the aesthetical valences in Greek mathematics. Besides problematizing diagrammatic reasoning, what both approaches share is the commitment to specificity concerning the genealogies of meaning across which they establish a continuous function.

Martin and Secor (2013:428) rightly point to this aspect by arguing that at stake in the geographical treatment of topology "is the clarity and precision of our theories of space". Their intervention addresses the potential of 'post-mathematical' topology to articulate spatial thought and offers a useful overview of the topological repertoires in geography, as well as a refreshing account on the relation between the topographical and the topological. Although in agreement with most of the points the article brings forward, we cannot help but wondering if topology and its conceptual substrate have ever been eminently mathematical or structuralist, as claimed by these authors. Thinking through the interplay between the axiomatic and the problematic, in the second part of this article, we will try to show how the spectrum of topological thinking has acted as a sort of rail-switch between and beyond different mathematical domains, by establishing new points of articulation with work conducted in physics and philosophy.

Along the calls for more inclusive approaches in current geographical appropriations of topology (Jones, 2009; Paasi, 2011b), we would suggest that the key perhaps lies in the sometimes painstaking translation process stimulated by the incorporation of ideas coming from mathematics, philosophy or other realms of thought. To evoke Gunnar Olsson's (2007:5) discussion of the abyss, the endless pit lying "between the worlds of being and understanding", in tackling the potential of topology we would thus like to resort to an exploration of the space in-between the visible and the less-visible, the axiomatic and the problematic. It is at the interface between these categories, we maintain, that some of the answers to our quest may indeed emerge, with reference to mathematics and, hopefully, geography as well. In the engagement with the early development of topology that follows, our analysis accordingly focusses on the passage and the overlap between several key 'binaries': the conceptual and the formal, the intuitive and the discursive, the classical and the postclassical, or what has occasionally been coined as the non-classical (Plotnitsky, 2002). After this genealogical exercise, in the final part we return to the potentially alternative roles that topology might play for work in geography and spatial thought in general. 
III.3. Topological trajectories: from the mathematics of space to the space of mathematics

The development of topology represents one of the pinnacles articulating the landscape of modern mathematical thought. Its genealogies of meaning go back as far as Greek philosophy and Plato's reflection on chora as a "protospace" (Plotnitsky, 2002:136), or Greek mathematics and the deployment of diagrams (Netz, 2003). However, the inception of topology has been broadly associated with the advent of the Scientific Revolution and its vibrant ethos, as a result of a time that opened up a fertile ground for tackling major questions in mathematics, physics and philosophy. Among the leading figures of this period, two great thinkers introduced what may be labelled as forms of proto-topology: Gottfried Wilhelm Leibniz and Leonhard Euler (Sachs et al., 1988). The first step in this direction was taken by Leibniz with his work on geometria situs and analysis situs in developing an ingenious method to study the properties of geometric figures through positional calculus (Durie, 2006; Knobloch, 2010). Along the major shifts in the development of geometry during the $17^{\text {th }}$ century, Leibniz's analysis situs was the first to introduce definitions and theorems of space, a move described by de Risi (2007:129) as "a truly remarkable innovation, for no definition of space can be found in Euclid's work or, as a rule, in any geometrical treatise prior to modern times". A few decades later, Euler developed the solution to the 'Königsberg Bridges problem' - evoking in his 1736 paper the possible connection with Leibniz's geometria situs (Sachs et al., 1988) - and later formulated the Eulerian characteristic of surfaces, widely acclaimed as pioneering contributions to the inception of graph theory and topology (Poincaré, 2010; Sandifer, 2010).

Introduced by Leibniz (de Risi, 2007), the term analysis situs was commonly used until the early $20^{\text {th }}$ century, when through the contributions of Henri Poincaré, David Hilbert or Hermann Weyl, and especially Luitzen Brouwer, the formal establishment of topology as a branch of mathematics met full recognition (Ferreirós, 2010a). Arguably, the most prominent figure that marked the early development of topological thinking was Bernhard Riemann. His ground-breaking work was connected to some of the most important shifts in the modern history of mathematics and physics, and fostered a new understanding of space in general, and of mathematical space in particular. Following up on Gauss' work, it was his stroke of genius to conceive of a new treatment of space through non-Euclidean geometry and complex functions, making possible new connections between strands of mathematical work previously alienated from each other. In his 1854 lecture On the Hypotheses which lie at the Bases of Geometry, Riemann set the ground for an ambitious research agenda onto the mathematical conceptualizations of space, corresponding to his formal introduction of the concept of manifold with a Riemann metric: 
"Measure consists in the superposition of the magnitudes to be compared; it therefore requires a means of using one magnitude as the standard for another. In the absence of this, two magnitudes can only be compared when one is a part of the other; in which case also we can only determine the more or less and not the how much [...] Such researches have become a necessity for many parts of mathematics, e.g., for the treatment of many-valued analytical functions; and the want of them is no doubt a chief cause why the celebrated theorem of Abel and the achievements of Lagrange, Pfaff, Jacobi for the general theory of differential equations, have so long remained unfruitful' (1882:56-57).

Riemann's visionary account of space importantly extended the discussion from the local-contextual character of geo-metrical properties to the convergence of various strands of thought in mathematics. In his approach, mathematical objects were defined "not in terms of ontologically pre-given assemblies [...] but in terms of concepts", each with their own "particular mode of determination" (Plotnitsky, 2006:194). Lars Ahlfors, a key exponent in the branch of complex analysis, regarded Riemann's topological reasoning as delivering "almost cryptic messages to the future", arguing that his conceptual account on manifolds "would defy any attempt at proof, even with modern methods" (Gray, 2010c:775). Along the lines of other important contributions to the development of topology - and in particular that of Felix Klein Hermann Weyl has pointed out this feature of Riemann's (2010:157) work, by arguing "that it is always the Riemann surface, not the analytic form, which is regarded as the given object".

The convergence of mathematical work mobilised by the early development of topology was outstanding. For David Hilbert and Cohn-Vossen (1990:289, emphasis added), "the theorems of topology have been found to be connected, despite their apparent indefiniteness, with the most precise quantitative results in mathematics, that is, with the results of the algebra of complex numbers, the theory of functions of a complex variable, and the theory of groups", remarking that "topology ranks among the most fertile and successful branches of mathematical endeavour". From the synthesis of non-Euclidean geometry by Gauss, Bolyai and Lobachevskii (Gray, 2010a) to Riemann's work on manifolds, the red threads of topology cut across some of the most important developments in modern mathematics, holding also a prominent position in the foundational crisis of the discipline that marked the end of the $19^{\text {th }}$, as well as the beginning of the $20^{\text {th }}$ century (Corry, 2010). This aspect is of great importance for our discussion on the interplay between the formal and the conceptual underpinning the spectrum of topological thinking, on which we return in the final part of this section.

A key figure to Deleuze philosophy, Albert Lautman regards the oppositions between mathematical notions as "superficial appearances masking much more profound relationships” (Dieudonné, 2011:xli). Thus, Lautman (2011) argues that the mobility 
of mathematical entities and their passage between poles of conceptual tension leads to outstanding developments in the discipline. As we shall see next, topology entirely fits this picture. The context in which these ideas have gained momentum was intimately linked to a particular mode of operation in scientific practice, through endeavours often aimed at questioning both the disciplinary boundaries and the conceptual substrate of mathematics, physics and philosophy. In light of these developments that marked most of the 19th century, Gilles Châtelet (2000) has underlined the role of allusive devices and thought experiments in engaging with some of the most ardent scientific debates. Drawing on the case of magnetic and electrical polarities, Châtelet (2000:75) provides a deep insight into the birth of 'centres of indifference,' which bring into existence new relational instances and, consequently, new 'envelopments' of the pair notion: "Symmetry is rediscovered as a producer of differences". According to Lautman's (2011) path-breaking analysis of mathematical ideas, the modus operandi of modern mathematics relies on the interplay between the formal and the conceptual ${ }^{1}$, through which new ways of passage and new 'points of articulation' take shape with reference to different domains. This perspective resonates with some of the most important developments mobilized by the spectrum of topological thinking, touching upon foundational issues as, for instance, the relation between the local and the global, the intrinsic and the extrinsic or the continuous and the discrete (Lautman, 2011).

Lautman discusses local versus global conceptions of space by resorting to the Theory of Relativity and the contrasting approaches in the works of Riemann and Klein. If the Theory of Special Relativity builds upon Klein's approach, where space is regarded as "globally homogenous" and having "the same constant curvature", the Theory of General Relativity is articulated by Riemann's geometry and draws on the local particularities of space, including the possibility of "variable curvature" (Plotnitsky, 2009:124; Lautman, 2011). The bridging point between these two positions lies in the works of Elie Cartan and Hermann Weyl on 'closed groups', with results attaining to the possibility of determining the (local) metric of a group from its global properties. Despite the structural differences between Klein's and Riemann's approaches concerning the closure of groups, the outstanding result obtained by Cartan via Weyl is that the global properties of a Klein group find their clearest expression through the local metric of Riemann space (Lautman, 2011). Consequently, at stake in this (partial) reconciliation of global and local conceptions of space are the profound implications of the duality defining the spectrum of topological thinking. This feature could also be identified in works exploring the interplay between intrinsic and extrinsic properties

\footnotetext{
1 Here Lautman draws on Hilbert's proof theory and on set theoretical methods, employed to explore consistency and completeness for the sets of axioms within mathematics (2011, pages 141-148)
} 
of mathematical objects. Accordingly, another fine example exposing the convergence of contrasting lines of thought pertains to the development of duality theorems.

The idea behind duality theorems refers to the study of mathematical entities through their counterparts (homology groups), by exploring the properties of given objects based on the relations established with their complementary structures. The initial contributions to duality theorems have drawn on set-theoretical methods, which in the early works on topology were considered to be in sharp contrast with the combinatorial ones (Alexandroff, 1961). The first steps in reconciling the two approaches were made by Camille Jordan and Henri Poincaré, through results initially regarded as disconnected from each other. In 1887, Jordan formulated the Jordan Curve Theorem, showing how a closed curve acts on the plane by creating two separate regions sharing a common boundary (Stubhaug, 2010). A few years later, Poincaré stated the Poincaré duality and provided a preliminary proof ${ }^{2}$ in his 1895 seminal paper Analysis situs, which marked the formal establishment of algebraic topology (Ferreirós, 2010b). While developing his duality theorem, Poincaré already intuited the great potential of a cross-breeding between set-theoretical and combinatorial methods in topological thinking. As Alexandroff (1961) has argued, both methods can be identified in Poincaré's early work, but their merge was not yet possible due to the existing tensions between the two strands of thought in topology and the lack of a general formulation for the concept of manifold.

Nevertheless, around the beginning of the $20^{\text {th }}$ century, Lebesgue's and Brouwer's contributions to Jordan's theorem lay down the premises to reconcile the two sides. According to Alexandroff (1961:31), "[i]n the face of these extreme positions, the monumental structure of Brouwer's topology was erected which contained - at least in essence - the basis for the rapid fusion of the two basic topological methods". Drawing inspiration from Brouwer's work and Poincaré's results, in 1922 Alexander formulated his own duality theorem, attaining to the contextual properties of mathematical objects through the analysis of their internal structure (Lautman, 2011). In line with the treatment of the local and global conceptions of space presented earlier, and the results of duality theorems, Lautman (2011:123) suggests that "[n]obody could indeed have suspected, before the development of algebraic topology, that the properties of internal structure discovered by Poincaré would someday explain the extrinsic situational properties expressed by Jordan's theorem". Consequently, the interplay of the ideal pairs of opposites evoked above, as well as their inherent sway between poles of conceptual tension expose the problematic dimension of topology or what Châtelet (2000:69) refers to as the "problematic

\footnotetext{
${ }^{2}$ His study extending the works of Riemann and Enrico Betti resorts to the analysis of Betti numbers invariants describing the connectivity of surfaces - in order to prove how the internal properties of a closed set could explain the properties of its complementary space (Lautman, 2011).
} 
latencies' available for opening alternative trajectories in the scientific practice. This perspective lies also at the core of Deleuze and Guattari's (1987) project, both in its engagement with mathematical notions and with the relation between minor and major science.

To further support our argument, our final example draws on the Uniformization Theorem, a major turning point in the early development of topology. Most contributions to its formulation have drawn on the study of analytic functions over two-dimensional manifolds, extending Riemann's results. Among the early works in complex analysis, the contrasting approaches of Riemann and Weierstrass in the treatment of functions exposed the polarization of the main streams of thought in the discipline (Ferreirós, 2010a). Along these lines, Poincaré's and Klein's contributions to the Theory of Automorphic Functions played a critical role in the later formulation of Uniformization Theorem (Weyl, 2010). This was Poincaré's first application of a nonEuclidean metric in the study of functions and, as Lautman (2011:62, emphasis added) argues, Poincaré "thought he was witnessing the accidental and almost inexplicable encounter between two orders of thought totally foreign to one another. Non-Euclidean geometry had seemed up until then to be a simple mind game that was only of interest to the philosopher, without being of any use to the mathematician, and it found itself to be essential in the uniformization theory of algebraic functions". On a similar note, despite his different view of Riemann's conceptual approach, Weierstrass has offered an essential contribution to the representation of functions on Riemann surfaces ${ }^{3}$ (Weyl, 2010). Consequently, Weyl (2010) was able to formulate a more general definition for Riemann surfaces ${ }^{4}$. According to Lautman (2011:135), through his new definition of the Riemann surface, Weyl was responsible for bridging Riemann's approach to "the spaces that he introduced in geometry and the surfaces that he introduced in analysis", contrasting with each other through their local and, respectively, global character. The final touch came from Poincaré and Koebe in 1907, when they independently furnished a general proof for the uniformization of analytic functions over Riemann surfaces (Gray, 2010b). As a result, the Uniformization Theorem led to the classification of surfaces into three main categories, rooted in Euclidean, spherical and hyperbolic geometry, emphasizing the predominantly hyperbolic character of most Riemann surfaces (Beardon, 2010). The Uniformization Theorem holds a special place along the genealogies underpinning the spectrum of topological thinking and, together with all the other examples discussed here, it offers but a glimpse at the swirl of ideas animating the

\footnotetext{
3 Weierstrass introduced the classification of functions according to the genus $p$ of the surface - a topological quantity expressing "the maximum number of independent closed curves that can be traced on this surface without dividing it into two separate regions" (Lautman, 2011, page 149).

${ }^{4}$ The new definition was based on the correspondence between the genus $p$ of the Riemann surface (number of holes) and the presence of Abelian functions (number of linearly independent integrals) on it (see Kleiner, 2010).
} 
conceptual space of modern mathematics. In turn, this brief genealogical exercise has hopefully proved productive in exposing the problematic dimension of topology and its potential to dis-locate established categories, by fundamentally undermining the stark separation between apparently opposed notions and canons of thought.

As pointed out above, the development of topology has played a fundamental role in the conceptual space of mathematics. We have seen this at work through the examples invoked so far, and it probably should not come as a surprise that the trajectory of topology was intimately connected to the crisis of foundations in mathematics. This is usually acknowledged as the period of the 1920s, marking the clash between Hilbert's formalist program and Brouwer's revisionist agenda. Ferreirós (2010a) proposes a more extensive analysis of the crisis, by evoking the groundbreaking developments of the late $19^{\text {th }}$ century and their sway over the foundational debates. Among these, we find Riemann's or Poincaré's pioneering works that fostered new connections between different strands of mathematical work. Henceforth, the end of the $19^{\text {th }}$ century is marked by debates around the role of nonEuclidean geometry and the foundations of mathematical analysis, or the development of set-theoretical ideas (Corry, 2010). The frictions between the different strands of work coupled to the foundational debates culminated at the beginning of the $20^{\text {th }}$ century with Cantor's formulation of set-theory and Hilbert's axiomatic program (Archibald, 2010), which opened new venues for the interplay between formal and conceptual thinking in mathematics.

However, it was with Brouwer's revisionist agenda that the debate over the foundations reached its peak. After earning a reputation of unconventional thinker through outstanding contributions to topology, Brouwer dedicated himself to establishing a new school of thought in mathematics through an eminently constructivist program, aimed at challenging the dominance of the formalist trend in the discipline (Van Stigt, 1979). Although received with enthusiasm by leading figures as Alexandroff, Poincaré and Weyl, his program based on 'intuitionistic mathematics' failed in the confrontation with the classical-formalist approach endorsed by Hilbert. Interestingly, in the wake of perhaps the most intense debate in the history of modern mathematics, marked by the clash of ideas but also the role of individual egos (Van Dalen, 2010), in the later refinement of his formalist program Hilbert acknowledges the importance of intuition as an essential component of mathematical work (Ferreirós, 2010a). In the preface of his book Geometry and the imagination, he states that "the tendency toward intuitive understanding fosters a more immediate grasp of the objects one studies, a live rapport with them, so to speak, which stresses the concrete meaning of their relations" (Hilbert and Cohn-Vossen, 1990:iii, original emphasis). On a similar note, Lautman (2011) endorses the inseparable character of intuition and reasoning as fundamental conditions of mathematics. To draw a parallel with Châtelet's (2000:66) 
discussion of amplum integrals, as devices describing the envelopment of all paths of a continuum "where they are virtually equivalent", it may be argued that this was also the case with the trajectory of topology across the landscape of modern mathematics. The unfolding of the paths defining the spectrum of topological thinking thus rested upon the passage between the formal and the conceptual, enabling a transit space turned by the swirl of ideas into a playground for imagination and possibilities to become actualized.

Before exploring the geographical valence of this statement, we would like to pin down some critical aspects pertaining to the trajectory of topology throughout the space of modern mathematics. Across our inventory of conceptual pathways we emphasized on several occasions the dual character of topology and the outstanding potential defining 'its binary'. In this context, by binary we specifically refer to the nexus between the formal and conceptual domains of topology. The latter concerns the problematic dimension, articulated by imagination and intuitive thinking, which by inducing tensions within the established orders of formal domains could stimulate the synthesis of new ideas and thus open new knowledge horizons. Lautman (2011:186) accordingly argues that mathematical reality "does not reside in the differences that separate the completed entities from the incomplete entities, perfect entities from imperfect entities. It resides rather in the possibility of determining one from the other, that is, in the mathematical theory that asserts these connections". This is indeed one of the most remarkable features defining the spectrum of topological thinking and its catalytic potential to establish points of articulation between seemingly opaque domains. To give it a more familiar twist, we could argue that topological thinking operates at the interface between the 'spatial' and relational proximity of mathematical entities. Hence, our exploration of the conceptual grounds on which contrasting approaches to space (and beyond) have been reconciled exposes a more subtle concern with how topological thinking has affected the very space of mathematics or, to put it differently, how the mathematics of space mirrored into the space of mathematics. This aspect is of critical importance for our discussion of the topological in geography and with this perspective in mind, we now return to our exploration of the surface and its obscure depths.

\section{III.4. Shifts and topological re-arrangements}

After going through this genealogical exercise, what are the lessons to be drawn for human geography? In our inventory of the conceptual work that informed the development of topology, we emphasised the role of imagination and intuitive thinking in exposing the problematic/conceptual dimension of topology and, consequently, its potential to erode the distinctions between apparently opposed notions and canons of 
thought. To iterate Deleuze's (1994) treatment of the problematic or Châtelet's (2000:69) account, "two different rhythms underpin the 'history of ideas': the one, completely discontinuous, of 'ruptures', 'paradigms' and their refutations, and the other of the problematic latencies that are always available for reactivation". As already highlighted in our retrospect of mathematical developments, we would like to argue now that the problematic of topology, by inducing tensions in the assemblage of established categories, could foster new shifts within the conceptual space of geography as well. However, this would require a reassessment of the classical fashion in which the topological has often been employed so far in stimulating imaginaries that drag along a whole array of clear-cut distinctions. As Bachelard (1994:218) points out in engaging with the dialectics of outside and inside, "[i]f there exists a border-line surface between such an inside and outside, this surface is painful on both sides". Thus, thinking critically through the surface/depth metaphor, as an expression of the interplay between the axiomatic and the problematic, there are three interrelated points that we would insist upon to make our argument more explicit.

The first refers to the proliferation of new imaginaries and conceptual motifs that has preceded, but also built upon, the 'topological twist' in geography. Consequently, it was argued on several occasions that "the new found relief of geography" (Allen, 2011:283) is not that new after all, and we are actually dealing with what has been coined by some, the topological return(s) in social and cultural theory (Paasi, 2011a; Phillips, 2013). This is precisely the first point we want to make with reference to Châtelet's account on the 'problematic latencies' pertaining to the history of ideas. The rush to put a squeeze on topology has led to an excessive engagement with its axiomatic (surface reading, if we may), by often othering the conceptual baggage that comes along with it, i.e. its problematic dimension. This has in turn stimulated a myriad of topological deployments, some more 'problematic' (not in the Deleuzian sense) than others, which reveal (paradoxically or not) that the very problematic dimension is absent in these accounts. In other words, différance takes its toll. The resulting 'topological trap' - which keeps establishing homeomorphisms (equivalences) between these axiomatic readings - is thus very difficult to escape, and perhaps this is why Martin and Secor (2013:431) wonder if topology has indeed "smuggled structure into spatial theory".

In line with Martin and Secor's observation, others have been attentive to this trend and have called for more inclusive approaches in the treatment of topology and its relation to the conceptual apparatus of geography (Jones, 2009; Paasi, 2011b). Employed in a rather classical fashion as 'other to the topographical' and, perhaps too often, in sharp contrast with the established categories in the discipline, the topological has clearly lubricated the geographical imagination, but at what cost? Arguably, the proliferation of topological imaginaries currently at stake in geography is 
primarily evocative of the 'eternal' return of the Other and seconded by its topological referents. Thus, instead of turning topology into the scapegoat of current geographical conceptualisations of space, we trust there might be other ways in which this could prove more resourceful, precisely by learning from the lesson offered by the trajectory and the effects of topological thinking in mathematics. Accordingly, the above treatment of the axiomatic and the problematic is key to the potential roles we envision for topological thinking in geography. And this leads to our second point.

Related to the above genealogical exercise and Elden's (2011) call for historical specificity, the main potential role we envision for topology pertains to the use of metaphors and their articulations within the conceptual space of geography (see Fall and Minca, 2013). Along the imperative to make mathematical and topological metaphors more explicit (Abrahamsson, 2012; Marques, 2004), we think that recovering the genealogical filiations through which these bridge the axiomatic and problematic would reveal their relevance for the various ontological positions they attempt to legitimise. For instance, the Möbius strip might be an interesting figure to engage with, but how did it come to be acknowledged as topological? Which were the strands of research that led Listing and Möbius to the discovery of this non-orientable surface, and how do those strands of research connect to the different conceptualisations of the Möbius strip within social and cultural theory? This is eminently an issue of observation and, as Châtelet (2000:101) points out, "[s]pace thus appears as a visible understanding and the understanding as an invisible space. The point is to clarify the link between the process of the individuation of the knowing subject and the process of articulation of the forms of the grasping of space". There is always a built-in tension into the exercise of mapping these genealogies that may turn into creative energy, to re-think along convergent or alternative pathways the status and potential of the spatial categories at stake. More importantly, there is a question of functional continuity or of how various topological imaginaries map onto the conceptual space of geography.

Our last point envisages a potentially foundational role for topological thinking in geography. In our exploration of the conceptual grounds on which contrasting approaches to space have been reconciled, we have emphasised the more subtle concern with how topological thinking has affected the very space of mathematics. In Riemann's pioneering approach to space "[e]ach neighbourhood is therefore like a small bit of Euclidean space, but the connection from one neighbourhood to the next neighbourhood is not defined and can be done in an infinity of ways" (Lautman, 2011:98). Echoing Lautman's analysis, Deleuze (1994:162) argues that in the treatment of the problematic "we must move to a geometry of sufficient reason, a Riemanniantype differential geometry which tends to give rise to discontinuity on the basis of continuity, or to ground solutions in the conditions of the problems". Grounding our attempt to rethink the role of topology for geography in the 'conditions of the 
topological' would, however, require a shift in focus. This issue lies at the core of our critical engagement with the surface/depth metaphor throughout the article. To shake-up this metaphor "out of habit of expression to actuality of expression" (Bachelard 1994:221-222), its critical deployment is aimed at expressing the dynamics between the axiomatic and the problematic in the treatment of topology and, in a more profound way, to undermine these distinctions by folding back topological thinking onto the dynamics it actually describes. In other words, applying topological thinking to the treatment of topology. Despite of having boiled underneath the 'surface' for quite some time, this is arguably a largely absent account in the current geographical engagements with the topological.

Martin and Secor (2013:422) stress that geographers' shared interest in topology is ultimately related to "a move to conceptualize the dialectic between continual change and enduring relations". Along previous calls for more inclusive approaches in the treatment of topology, they further argue that "[t]opology's 'post-mathematical' potential resides (...) in how it is reworked through postructuralist theories of space" and that to "move spatial theory in geography forward, our engagements with topological thinking need to build on one another, rather than to produce an endless proliferation of singular deployments" (Martin and Secor, 2013:435). Saluting their argument, we think that the fundamental role we envision for topological thinking in geography runs at a deeper level. In line with our genealogical exercise, the shift in focus invoked earlier relates to a post-classical or non-classical enterprise (Plotnitsky, 2002) of applying topological thinking to how topology is reworked through poststructuralist theories of space'. More precisely, this calls for a closer scrutiny of how geographers' empirical and theoretical engagements with topology map onto, patch or alter the conceptual space of geography. To give it a Deleuzian twist, this concerns how various topological deployments de-/ re-territorialise the conceptual space of geography and the related imaginaries it informs. As with mathematics, this may allow topological thinking to be mobilised as a rail-switch in exploring various affinities and tensions within human geography's patchwork of 'canonical' and emergent imaginaries. In reworking the interplay between the former and the latter, 'naturalizing a single typology of topologies' might prove of little use (Vasantkumar, 2013:929). Instead, a more careful consideration of the problematic calls in for topological approaches that could expose new fixed points and shifting spatialities by attending to the 'negative space'/ 'residual surplus' on which both more traditional and state-of-the-art analytical tools rest upon. We elaborate on a topological logic of fixed points and on the reworking of various geographical proximities in a forthcoming article, based upon an empirical account exploring notions of creative reuse with reference to forms of religious ritual in Volgograd, Russia. 
Tackling this dual condition which underpins the spectrum of topological thinking, we suggest, would enable geographers to get a better hold on how the axiomatic and problematic unfold along/across 'our' poststructuralist theories of space, as well as how these interfere with the realities they try to put a squeeze on. Pushing the topological beyond its status as a mere category, would allow it to become a dynamic operator in revisiting the "ontological underpinnings of the very concepts at issue" (Malpas, 2012:5). And yet, to establish new points of articulation through which geographical categories and new imaginaries (topological or not) could coexist and prove more lucrative in pressing against the 'unknown'. Thus, topological thinking could add indeed a new dimension to conceptual work in geography, in carving out the genealogies of meaning that would sustain the functional continuity of spatial and not-so-spatial categories, and their articulations within new knowledge horizons. Ultimately, this shift in focus might still produce a few puzzling 'returns' and this has probably to do more with geography's own legacy. Underneath the rubble of battered structures and forgotten pasts, there might still lie some 'problematic latencies' on which to cast a new light, an aspect emphasised by Keighren et al. (2012) in their discussion of canonical geographies. To use Serres' claim concerning the history of sciences, it might prove useful also for geography, in the endless process of reinventing itself, to embrace the perspective of "a radical contemporaneity or copresence of the archaic and the contemporary" (Serres and Adkins 2012:369).

\section{III.5. Coda}

By deploying the surface/depth metaphor as an expression of the dynamics between the axiomatic (formal) and problematic (conceptual) dimensions of topology, in this article we have suggested some potentially alternative ways of mobilising topological thinking in human geography. Our intervention has drawn upon the observation that geographical appropriations of mathematically-informed notions have been less attentive to the problematic dimension of topology. Consequently, through a genealogical exercise we have tried to expose the pivotal role held by the interplay between the axiomatic and problematic in the early development of topology and its establishment as a formal branch of mathematics. In tackling the question of how this exercise could prove useful for geography's commitment to topology, we insisted on three interrelated points. First, we argued that the predominant concern with the axiomatic dimension of topology has inhibited the engagement with its 'problematic latencies' and their potential to foster new points of articulation within the conceptual apparatus of geography. Second, we emphasised the catalytic effect that topological metaphors could have in exposing the interplay between the axiomatic and the problematic, and as such to articulate more coherent topological repertoires. Third, we envisioned a potentially fundamental role for the topological, pertaining to a shift in focus that 
would transcend its status as a mere category. Arguably this would allow the topological to act as a dynamic operator across the conceptual space of geography in rethinking the connections between established toolsets for spatial analysis, as well as their affinities with emerging imaginaries. Furthermore, such a move might enable geography to re-engage with its past in the endless process of re-inventing itself. Acknowledging the limitations of our attempt to re-imagine the role of topological thinking in geography, both in its engagement with philosophy of mathematics and various interventions in social and cultural theory, we nonetheless hope to have patched things up with the "topologie samvage" and the "world of continual questioning" that comes along with it (Thrift 2008:120, author's emphasis). At least for now... 


\section{IV.The floating churches of Volgograd: River topologies and warped spatialities of faith*}

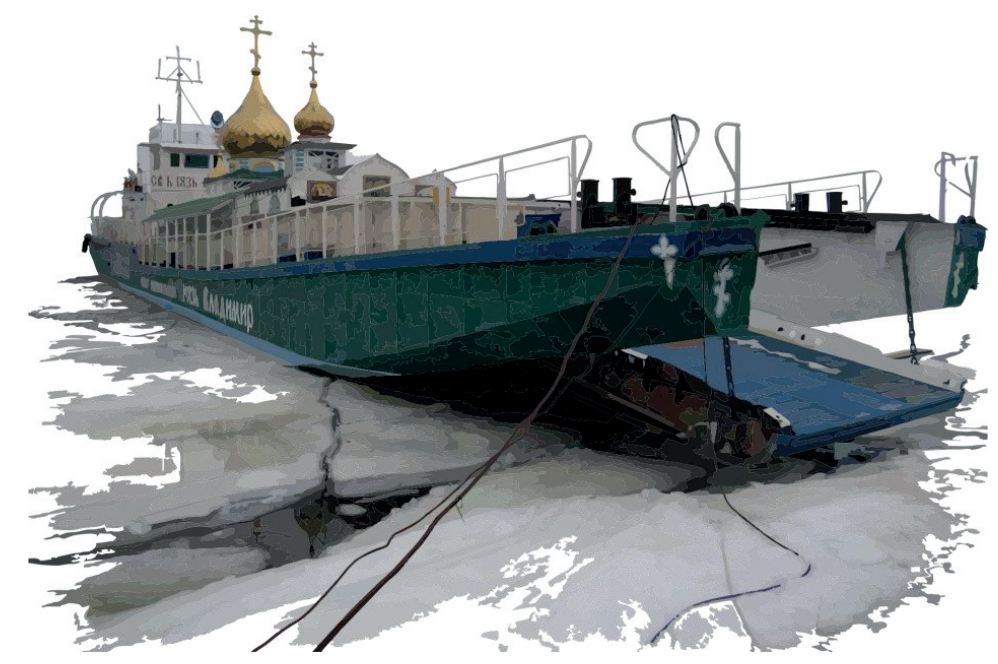

*This chapter is based on the initial version submitted to Transactions of the Institute of British Geographers - currently amended according to the referee's reports:

Barba Lata I and Minca C (under review) The floating churches of Volgograd: River topologies and warped spatialities of faith. Transactions of the Institute of British Geographers. 


\title{
IV. The floating churches of Volgograd: River topologies and warped spatialities of faith
}

\begin{abstract}
This article explores the concept of creative reuse as an alternative modality to interrogate the materiality of things and their documentary sway beyond the immediate affordances dictated by circumstances of disposal or dissolution. Drawing on an amphibious ethnography of the Volga and Don riverscapes, we evoke the case of the floating churches built to support the revival of faith practices in the Volgograd oblast after the collapse of the Soviet Union. In attending to their impact in warping various temporal and geographical proximities, we suggest that their workings rely on topologies of fixed points and shifting spatialities, animated by forms of religious ritual and related creative manifestations. Through recourse to questions of materiality, mobility and affect we argue that creative reuse interventions provide productive ways of exposing and altering the residual surplus on which both things and processes of place-making rest upon.
\end{abstract}

Keywords: floating churches, creative reuse, topology, archiving, Volgograd

\section{IV.1. Introduction}

Every year around the end of July, the city of Volgograd hosts a little known but rather spectacular festival, where live music, poetry and dance performances mingle with religious practice in a unique manner. Organised in Kirovsky district by the Volga River embankment, the three-day festival celebrates the memory of Russian bard Vladimir Vysotsky, an iconic artist of former Soviet Union (Lazarski, 1992). The event is hosted by the local entrepreneur Vladimir Koretsky and includes a diverse pool of performers, of various nationalities, ages and backgrounds. An interesting feature of the festival is its experimental set-up, both in terms of performances and spatiotemporal layout, which allows for the eclectic mix of creative manifestations to fuse with manifold religious motifs through a great deal of improvisation. While attending the $11^{\text {th }}$ edition, back in 2013, we witnessed how creative and spiritual encounters are staged through a succession of ceremonial moments across various 
sites bearing the mark of Vysotsky's popularity. The opening event took place at the local yacht club called Parus, built as a tribute to the Russian bard's oeuvre. The club's main building provides a panoply of artwork, from murals depicting scenes of his artistic career, complemented by the lyrics of his songs and poems, to an impressive display of astrological and Orthodox-religious motifs. As part of an already established tradition, the event started with a religious ceremony held for blessing the venue and participants, including also a procession along the river quay to Vysotsky's memorial and the location of the festival stage. The prayer service culminated in a spectacular moment, when an airplane carrying sacred relics circled the area several times, marking the transition to the organiser's welcome speech and the beginning of live performances on stage.

In line with the mix of performances and the event's tempo, the spatial arrangement of the festival setting presented some distinctive features as well. While the audience was concentrated on the gentle slope of the riverbank, where long benches had been laid out, the festival stage was floating on the Volga. Around twenty meters upstream, flanking the left side of the stage, a more silent presence offered an almost surreal sight. A ship-like-no-other laid moored perpendicular to the riverbank, as if it was there to protect the floating stage from the strong river currents. Marking the main axis of the ship, three gilded onion domes carrying the Orthodox cross emerged from what appeared as a chapel structure (figure 2). We were told by our hosts that the ship was actually a floating church, named Saint Vladimir, which travelled on the Volga River to provide religious services to churchless communities. Fascinated by the look and the function it performed, we attempted to find out more about the story of Saint Vladimir. So we learned that, apart from providing religious functions to villages within the oblast ${ }^{5}$, the floating church served as a mobile stage for charity concerts, as well as an open canvas for different kinds of representation: while being a mobile sacred space, it was also a site for creative performances. However, to our surprise, we soon realised that Saint Vladimir was not the only ship of its kind in the Volgograd oblast and that there existed a legacy connected to what was acknowledged as the 'flotilla for God'6.

Inspired by some remarkable developments rooted in the history of mobilities as articulated by Russian Orthodoxy, the floating churches distinguish as an innovative response along processes of identity formation and place-making in the Volgograd oblast. Built after the collapse of the Soviet Union to support the revival of faith practices, they had an outstanding impact in patching up a constellation of sites completely

\footnotetext{
${ }^{5}$ Administrative division in Russia.

${ }^{6}$ The Volgograd floating churches were first referred to as 'flotilla for God' by Father Werenfried van Straaten, the founder of Aid to the Church in Need: http://www.acnuk.org/news.php/165/russiachapel-boat-sets-off-on-ecumenical-voyage
} 
stripped of religious infrastructure. With their journeys, the floating churches have reanimated the sediments of a contested order - symptomatic to the oppressive campaigns previously directed against religious life - by exposing their operative geographies to alternative spatiotemporal conditions of encounter and new forms of attunement. We thus proceed from one of the striking albeit innovative features that the floating churches arguably share, their rudimentary character. Jury-rigged hybrids made of salvaged ships and all-sorts-of-other-things, some more tangible than others, we argue that the floating churches emerge as an expression of creative action, or rather creative reuse, seeping through various degrees of materiality and affects. Their travelling cycles have in fact weaved a whole range of absences and presences into topological arrangements that contract and expand with the shifting spatialities of religious rituals and the meanings attached to mooring sites.

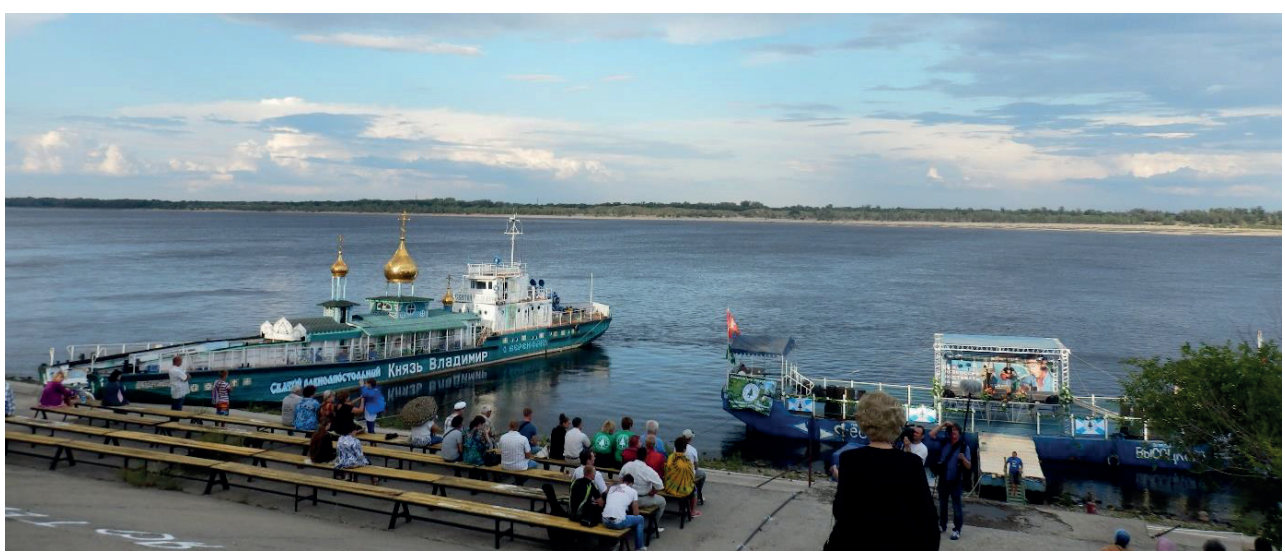

Figure 2. Saint Vladimir floating church (left) and the floating festival stage (right). Source: The authors

Taking up Dwyer et al.'s (2013) call for a more careful consideration of religious creativity and interventions that have sought to rethink the status of archival practices in geography (Cresswell, 2012; DeLyser, 2015; Dwyer and Davies, 2010), we dwell on notions of creative reuse in enacting alternative archives, which coil up various materialities and affects into mobile cosmologies/theologies that, beyond reworking the connections between past and present conditions though their documentary sway, inform also future trajectories. By evoking a series of historical mobilities and contingencies, we treat creativity as a cumulative process of attending to the 'residual' surplus of things beyond the immediate affordances dictated by circumstances of disposal or dissolution. Operating within this imaginary, we explore questions of materiality, mobility and affect in unveiling a topology of fixed points and shifting spatialities animated by forms of religious ritual and related creative manifestations. The paper is accordingly structured into two main sections. The first discusses creative reuse as an alternative modality to interrogate the materiality of things and as 
a potentially lucrative perspective in taking work on archival practices further. The second explores creative reuse in relation to the establishment of the floating churches in Volgograd and, consequently, how their impact is scored across various spatiotemporal conditions and functional registers. We conclude with a reflection on the possibilities that topologies of creative reuse might open up for different strands of work in geography and beyond.

\title{
IV.2. Geographies of creative reuse
}

\begin{abstract}
"It was a geometry book, which he had to hang by strings on the balcony of his apartment in the rue Condamine; the wind had to go through the book, choose its own problems, turn and tear out the pages. Suzanne did a small painting of it, 'Marcel's Unhappy Readymade'. That's all that's left, since the wind tore it up. It amused me to bring the idea of happy and unhappy into readymades, and then the rain, the wind, the pages flying, it was an amusing idea..." (Cabanne and Duchamp, 1987:61)
\end{abstract}

Marcel Duchamp's provocative artworks putatively inhabit a realm informed by counter narratives of creative reuse that have sought to erode the aesthetical fundaments and elitist pretensions of conventional art. As his distinctive signature, the readymades ${ }^{7}$ are often evoked as expressions of functional and temporal displacement (Moore, 2011) that "flow into a principle of universal metamorphosis" "[u]ndermined in the process is the idea of art as communication, enhanced instead the idea of art as life" (Olsson, 2007:162). In the fragment cited above, Duchamp recalls the gift made to Jean Crotti upon marrying his sister Suzanne, a geometry book that he playfully describes as an 'unhappy readymade'. Once suspended in the open, the geometry book becomes a mere supplement to the vortical forces of the elements, consumed by its otherwise 'negative space'. Given the least acknowledged interest Duchamp has manifested in the mathematics of higher-dimensional spaces and Einstein's work (Henderson, 2009), his reference to the geometry book as an 'unhappy readymade' arguably provides an interesting key to tackle the dynamics behind the making and unmaking of things. As Daniel Miller (2011:23) points out, in moving beyond the taken-for-granted status of things "it is only when the juxtaposition or material is distinctly odd that we are shocked into an awareness of the underlying technology".

Over the past decade or so, questions of matter and materiality have animated geographical debates around a broad range of material-affective imaginaries, aimed at exposing a world of mixtures and flow, of labile elements shifting between various

\footnotetext{
7 Ordinary objects elevated to the status of art. 'According to Duchamp, the artist's choice of a readymade should be governed not by the beauty of the object but by his indifference towards it; to these ends it could be selected by chance methods, for example by a predetermined weight or at a predetermined time' (Gale, 2009): http://www.moma.org/collection_ge/theme.php?theme_id=10468
} 
states and predisposed to all sorts of affinities (Adey, 2015), attunements (Stewart, 2011) and turbulences (Cresswell and Martin, 2012). Extruded from such elemental mobilities, bodies mingle through complex processes of circulation generative of affective atmospheres and spatiotemporal conditions of various intensities, rhythms and momenta (Anderson, 2009; McCormack, 2014; Merriman, 2015). As Nigel Thrift (2008:19) has convincingly argued in line with Latour, "there is every reason to believe that we are surrounded by innumerable hybrids, only a few of which we have named and even fewer of which we can claim to understand". Undoubtedly challenging, with the emphasis on a generous reading "as variously turbulent, interrogative, and excessive, materiality is perpetually beyond itself” (Anderson and Wylie, 2009:332). The 'stuff of matter', or what Ingold (2007:10) refers to as materials, "always and inevitably win out over materiality in the long term", a perspective growing out of various processual philosophies that attend to the vortical logic behind the making and unmaking of things (Bennett, 2010:119; also, Serres, 2000). However, along these lines of enquiry, Ash and Simpson (2014:15) warn over the enthusiastic adherence to questions of matter and materiality that on the flipside risk turning into "no more than a generalized metaphor that ignores the objects that actually appear in a given moment". Their intervention indeed raises a series of relevant questions on the possibilities to grapple with the status of things while coupled to process of varying dynamics. In this regard, the temporal and functional registers of things emerge as key dimensions.

In the article The death of great ships Mike Crang (2010:1086) provides an intriguing account on shipbraking in South Asia by evoking the time-image of decommissioned ships as waste, "products at the end of their lives, dumped, discarded, and being dismantled (...) that stresses their undoing and unbecoming". The focus on unbecoming arguably strikes the nub of a largely underrated matter, i.e. the destructive dimension coiled into things as a result of the interplay between their materiality and functional registers (Gregson et al., 2010). In crude terms, contrasting it to a vitalist progression of becoming, unbecoming would equate death or, in a more nuanced manner, the conditions of a process of dissolution. Thus, unbecoming heralds the end of a phase, the unveiling of the vulnerable condition of things while they are consumed within the ecologies that rendered them redundant. In maintaining this overtone, attending to the problematic of becoming-unbecoming and their inseparable conditions translates into a topology of 'fixed points' and shifting spatialities. In other words, novelty and redundancy, appropriation and disposal are co-emergent with the functions that enable the thingness of things across given sets of spatiotemporal conditions. Topologically, fixed points emerge as markers of recursive functions ${ }^{8}$. Yet, despite the

\footnotetext{
${ }^{8}$ Brouwer's Fixed Point Theorem reformulated by Richeson (2011):

http://divisbyzero.com/2011/01/12/beautiful-theorems-about-dynamical-systems-on-the-plane/
} 
counter-intuitive reference to fixity, they are dynamic rather than static entities subject to various couplings that would sustain the functional continuity of a topological domain. Building upon the pioneering works of Henri Poincaré and Luitzen Brouwer, fixed point theorems have played an important role in the early development of topology through their non-constructive character, meaning that "they establish the existence of a fixed point rather than defining one or telling you how to find it" (Bergelson, 2010:693). The conceptual substrate of these ideas, particularly in capturing the dynamics between singularity and multiplicity, is traced back by Gilles Châtelet (2000) to the dawns of experimental electromagnetism, while Michel Serres (2006:40) invokes the Keplerian revolution in accounting for the displacement of the centre through multiplication. Châtelet (2000:75) draws on the case of magnetic and electrical polarities to emphasise the elusiveness of the origin point, described as a shifting indifference centre' that "makes it possible to envelop a whole fascia of virtualities without mixing them up".

In line with John Law's (2002:92) discussion of object alterity, through their functional registers things branch out into multiple 'spatial conditions of im/possibility'. Furthermore, these could be subject to temporal proximities that transcend a chronological reading of time or what Serres describes as a percolation model, "a complex surface, conveying wormholes of sheer acceleration, bottlenecks of stoppage or equilibrium, zones of stationary values, several fragmentations" (Serres and Adkins, 2012:377). Accordingly, one among manifold instances through which the temporal proximities evoked above are manifest pertains to notions of creative reuse and their documentary sway over emergent materialities. Things and the affects they generate have potent afterlives (Thrift, 2008:9) and, as emphasised by Ash (2014:7), "it is [often] the afterlives of affects that have the biggest impact on the beings exposed to them". Creativity here emerges as a form of attending to the residual surplus things drag along with them through the complex processes and contingencies at stake in their becoming and unbecoming. By partially overlapping, overriding or even fracturing the functional status and materiality of things, interventions pertaining to creative reuse enable hybrid formations that often reveal alternative conditions of experiment and attunement. Taking a more radical view on questions of usage and waste, improvisation and intentionality, various strands of work have challenged the linear treatment of creative reuse. For instance, Hale and Barber (2012:166) explore the case of steampunks as retrospective futurists that "reshape and refine elements of prior temporalities into refurbished forms in a process known as upcycling", defined by the authors as "the creation of forms from waste materials that attempt to be of a higher quality and more sustainable nature than the compositional elements from which they were derived". As such, steampunks negotiate multiple temporalities through their costumes and contraptions, which "are animated by and embedded within narratives 
of alternative realities, counterfactual pasts filled with retrofuturistic fashion and steam-powered technologies" (Hale and Barber, 2012:167). On a different note, in documenting forms of spiritual encounter within the New Age movement, Julian Holloway (2003:1967-1968) points out that "seemingly everyday objects are patterned into a relational topology of senses, movements, rhythms, and affective action' that enables a "field of emergent sacralisation".

Echoing the early techniques of collage and assemblage developed in Apollinaire's poetry and Braque's works, as well as Duchamp's readymades, found objects had been scoring among the most prolific (re)sources in modern art over the past century or so $^{9}$. In moving beyond the immediate affordances of things, temporal and functional displacements enact topological arrangements that recast the absences on which taken-for-granted materialities rest upon, as with Duchamp's 'unhappy readymade' caught in the swirl of the elements. The readymade thus occupies a space-in-between, since by being "relocated from one context to another, its identity becomes unfixed in the process - it is not at home when it is made into art, nor is it ever comfortable again when returned to its usual environment" (Moore, 2011:398). Accordingly, William Viney (2014:60-61) evokes Mark Dion's collaborative project the Tate Thames Dig, a large cabinet put on display at Tate Modern, which gathers a panoply of found objects made available by wind and tide that the artist together with a team of archaeologists and volunteers has recovered from the Thames riverbanks. Through its odd and unstable character, Dion's project provides a strong statement toward archiving and display practices, for "objects in the cabinet are carried by complex, continuous and fluid process of cycling in and out of the times of use and waste, attended by and expressed through assembly and disassembly, preservation and dispersal, retrospective legibility and dust" (Viney, 2014:179). Tim Cresswell (2012) highlights the messy and permeable character of archives by focusing on gleaning practices and how they piece together all sorts of memorabilia and sites, in enacting alternative value regimes and histories of the Maxwell Street market in Chicago. Adding another register to this body of work, Dydia DeLyser (2015:209) addresses the potential of seemingly residual materials accumulated over the research process, in this case a personal collection of kitsch souvenirs, which had an important impact in 'shaping social memory' in a 'broader community'. According to these accounts, creative reuse thus qualifies as a technique and process of hacking into or even overhauling the materiality of things through the creative reinterpretation of their functional registers and the branching out of their documentary capacities. To return to the case of shipbreaking and the emergence of alternative material flows, Gregson et al. (2010:853) point out that attending to conditions of dissolution "insists on seeing that things are assemblages, ontological conjunctures of stuff, materials,

\footnotetext{
${ }_{9}^{9}$ MOMA - Objet trowvé entry: https://www.moma.org/collection_ge/theme.php?theme_id=10135
} 
brought together and held together, but also coming apart and wrenched asunder' in which 'their value is not as a thing [anymore] but a function of their materialities and their ability to multiply and mutate".

\section{IV.3. River topologies and warped spatialities of faith}

The story of Volgograd's 'flotilla for God' unfolds along the cut of a complex set of narratives, contested landscapes and hybrid materialities taking shape at the interface between land and water. Even at a first glance, the floating churches of Volgograd appear as a fascinating case. However, what arguably makes them really stand out as innovative is their improvised character and capacity to carry more content than the Plimsoll marking would let it show. They distinguish at once as both singular and multiple in compensating for the lack of religious edifices within their operative areas. The creative reinterpretation of their functional registers has enabled them to establish anchoring points within different practices, across and beyond the Volgograd oblast, as well as to bridge tradition and novelty in an ingenious way. Built rather recently, they also retain the patina of older developments in a mixture which enhances a wide spectrum of resonance and 'affective qualities' (Anderson, 2009). As we shall see next, they emerge as an expression of the 'potent afterlife' of things (Thrift, 2008:9), through trajectories that seem to mirror the intricate topologies of the rivers they navigate upon. Tackling these meandering pathways in "seeking to document challenging labile environments of water and air" (Dwyer and Davies, 2010:89) would require a journey upstream, a mapping of confluences and tributaries toward the source(s) (Serres et al., 1997:17). Thus, in order to expose the operative topologies of the floating churches, we built upon an amphibious ethnography of creative reuse on the Volgograd riverscapes, which unfolds along the temporal and functional displacements that have informed their materialities and travelling cycles. Our investigation has drawn upon the collection of local stories, interviews, archive materials and on-site direct experiences in documenting the making and impact of the floating churches. In line with our previous intervention on the application of topological approaches to geographical analysis (Barba Lata and Minca, 2016), we interrogate the dynamics behind the reworking of various geographical proximities that, on the one hand, stabilized the functional registers of the floating churches and, on the other, enhanced their capacity to warp their operative areas. We accordingly argue that the relative stability of their functional registers relies on topological arrangements of fixed points and shifting spatialities that allow for their mobile materialities and operations to be performed. 


\section{IV.3.1. The Pirate metamorphosis}

The inception of the floating churches originated in a series of entwined factors particular to the Russian context for most of the nineteenth century and up until the major shifts in political and religious life brought by the Bolshevike Revolution of 1917. Among these factors were the mobilities articulated by church missions but also by military campaigns and their use of "movable, field churches" and "temporary worship houses" (Sidorov, 2000:215). Another important aspect evoked in various travel accounts and conveyance reports concerned the inconvenience of land transportation, highlighting the potential of the extensive network of waterways as a viable alternative (Moberly, 1862). In tracing back some of the creative reuse interventions that have inspired the making of the floating churches in Volgograd, we proceed from the convoluted story of a ship that was subject to multiple functional displacements, including its becoming and unbecoming as a place of spiritual encounter. The first documentary evidence of an initiative to build a floating church dates back to the turn of the twentieth century. In 1903, the Astrakhan-based merchant N.E. Yankov proposed to the local diocese to support the construction of a mobile church to offer religious services to communities and fishing cooperatives active on the lower Volga (Usov, 1993) ${ }^{10}$. Travel logs of the period provide accounts on the sheer size and importance of these communities for the local economy, as in Rowe's (1870:24) $A$ Journey on the Volga:

"We were taken on the next day by steamer to visit one of the fishing villages. These are called utschiugi, from the Tartar word utschiug, a fish-dam. They consist of a hundred huts, or more, together with curing and store houses, and sheds for making caviar and isinglass. During the fishing seasons (...) about 20,000 strangers assemble, in addition to the regular population engaged in the trade."

Received positively by local clergymen, Yankov's proposal induced the diocese, a few years later, to appoint a special committee to handle the task. Due to insufficient resources, the committee concluded that having the floating church built from scratch would be unfeasible. Consequently, it opted for the conversion of an existing ship that met the technical requirements to accommodate the new function. Under the patronage of the diocese and the officials of Astrakhan province, between 1908 and 1910 the committee collected donations for the construction of the floating church and conducted a thorough examination of more than thirty ships. After having raised enough funds, in January 1910 the tug-passenger steamer Pirate from Astrakhan merchant P.M. Minin was purchased and converted into Saint. Nicholas floating church

\footnotetext{
${ }^{10}$ This account on Saint Nicholas steamer builds upon information reported in A. Usov's article Floating Temple, Formerly "Pirate" (Plavuchikh khram, byvshiy "Pirat") - and triangulated with other historical materials cited in text.
} 
(Usov, 1993). At that time, the ship had already been in service on the Volga for roughly half a century. Initially owned by the Volga Steam Navigation Company, it was part of an order - commissioned in 1858 to London-based shipbuilders Ravenhill, Salkeld \& Co and Samuda Brothers - for the delivery of four shallow-draft steamers to operate frequent passenger trips between Kazan and Astrakhan (Talygin, 2011). Given the size of the Marienski canal locks - located on the main inland navigation route most ships had to be delivered in parts and assembled at local shipyards (Moberly, 1862). This was also the case with the steamer purchased by the diocese, which was put together at Kriushsky harbour in the Simbirsk province, where the workshops and main winter station of Volga Steam Navigation Company were located (Oliphant, 1854).

For almost fifty years the ship had been navigating on the route Kazan-Astrakhan under the name Kriushi. After being sold to Minin in 1908 and renamed Pirate, it mainly covered the lower parts of the Volga and the shallow waters of the transfer area by the Caspian Sea, from where larger ships could not navigate further inland (Talygin, 2011). Having the appropriate size made it an ideal candidate for the committee in search of a reliable vessel, capable of reaching the communities often located by the backwaters and narrow branches of the Volga. The Pirate metamorphosis into a floating church took only three months. The conversion project was entrusted to the diocese architect Karyagin, with construction works running under the committee's supervision. Karyagin had the challenging task of figuring out how to adapt the available space to the religious function, while including a reasonably-sized refectory and a sickbay, as well as having enough cabins for a crew of eighteen. The steamer's straight bow allowed him to come up with the ingenious solution to accommodate the church space at the front of the hull, by placing the narthex, nave and sanctuary underneath the main deck. Above deck, following the narthex perimeter, Karyagin imagined a rectangular superstructure marked by five gilded towers, which included the space of the choirs and the main dome. In-between the paddle boxes, on the upper deck, the church received a belfry chapel ending into an imposing gilded tower which, added to the ones guarding the narthex and the sanctuary, must have provided an impressive sight (figure 3). The additional spaces of the refectory and sickbay were located at the stern, above the main deck, while the crew cabins were spread all across the ship. After roughly three months of intense efforts to entirely change the ship's layout, Saint Nicholas floating church was consecrated on April 11th 1910, during a major ceremony held by the Bezzubikova pier in Astrakhan (Usov, 1993).

On April 16 ${ }^{\text {th }}$ Saint Nicholas took its maiden voyage from Astrakhan and upon arrival at the Caspian Sea the priest performed the Divine Liturgy for the sailors, merchants and fishermen celebrating Easter away from home. According to archive reports of the period, during its first year of service the floating church travelled around 4000 
miles, visiting villages and fishing cooperatives scattered across the Volga Delta, and the ships stationed around the river mouth (Usov, 1993). Apart from providing the religious service, the crew offered free meals and medical assistance to those in need, which made Saint Nicholas very popular well beyond the Orthodox denomination. After a few years in service, it already had an outstanding impact in the life of communities living by the Volga. This had apparently led Tsar Nicholas II to acknowledge its importance and donate in 1912 his yacht Marevo to the Alexander Nevsky temperance society from Saint Petersburg, to establish a second floating church on Neva River (Ivanov and Suprun, 2006). However, after operating for five years on the Volga, in 1916 Saint Nicholas ceased to perform its regular voyages and was put on sale by the diocese. The reason invoked was the lack of financial resources to sustain its activity. The announcement met lots of resistance from communities in the province, who initially mobilised to keep the church operational. In response to these reactions, the diocese committee formerly responsible for building the floating church presented a report where it emphasized the poor technical condition of the vessel and the high costs incurred by keeping it in service. Nonetheless, after being decommissioned from religious service and sold, the steamer continued to be used as a freight carrier on the Volga until 1918 and afterwards dispatched as a rescue ship in Baku, following up Lenin's decree to nationalise the fleet ${ }^{11}$.

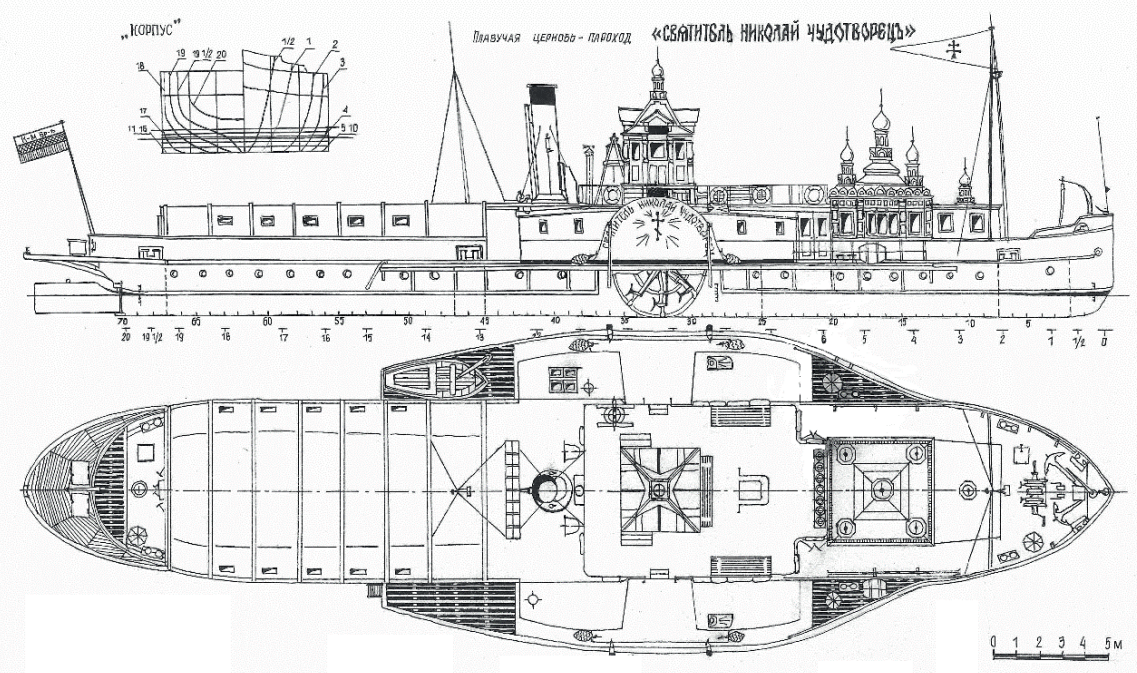

Figure 3. Starboard elevation and plan view of Saint Nicholas floating church. Source: Reproduced with kind permission of Nikolaeva Oksana

${ }^{11}$ L. Sevostyanova Temple on water/History of the floating temple "St. Nicholas", 1910-1915 (Khram na vode/Istoriya plavuchego khrama "Svyatitel' Nikolay Chudotvorets", 1910-1915 gody):

http://urokiistorii.ru/node/52234 
Although a short-lived development, the case of Saint Nicholas emerges as an expression of creative reuse through the ingenious alteration of its materiality and functional registers that enabled it to act as a shifting centre of religious activity for the communities located on the lower Volga. To draw a parallel with Duchamp's concept of readymade, the metamorphosis of the passenger/church/freighter/rescue ship has been actualised through a series of temporal and functional displacements that unveiled the intermeshing of its operative topologies. The resulting residual surplus as an inherent condition of the 'not-yet become' (Anderson, 2006:695) would later manifest through the afterlife-affects generated by the making and unmaking of the floating church in Astrakhan. For Thrift (2008:8), "as practices lose their place in a historical form of life, they may leave abandoned wreckage behind them which can then take on new life, generating new hybrids or simply leavings which still have resonance". Concerning our analysis, the fact that the loss of Saint Nicholas took place in the context of the First World War and coincided with the gales of the Bolshevik Revolution should not be neglected.

\section{IV.3.2. Mirroring (bi)stories and new beginnings}

In the neighbouring provinces of Astrakhan, Rostov and Tsaritsyn - for over three centuries the fief of the Don Cossacks - the Bolsheviks seizure of power and the Civil War that followed brought additional measures to the mass terror campaign conducted across the country to reinforce state authority. The Cossacks allegiance to the monarchy and the support offered (in most cases) to the Whites during the Civil War, have turned them into a target ethnic group for the Soviet regime. Legitimised by the state of war, countermeasures to acts of rebellion led to "the blurring of the line between actual and potential resistance" as "a constant feature of Soviet terror" (Netz, 2010:162). Among these repressive policies, the decossackisation campaign initiated in 1919 sought "to eliminate the Cossacks as a distinct group" together with their strongholds (Olson, 2004:161). Drawing a parallel between decossackisation and dekulakization, which took place a decade later with Stalin's collectivisation reform, Holquist (1997:131) points out that:

"[T] he administrative partition of the Don Territory had less to do with geographic reorganization than with population management. For much of 1919, the Soviet state planned to append portions of Tsaritsyn province and the Donets Basin, along with their populations, to the Don Territory. The explicit goal was "to dilute" the Don's Cossack presence through the introduction of a more reliable worker "element". By 1919, seeing and acting upon "elements" in the population had become endemic."

Mass arrests, deportations and executions were complemented by the systematic destruction of many Cossack settlements around the Don and Kuban provinces 
(Skinner, 1994). Due to its strategic location, Tsaritsyn - later to become Stalingrad and a symbol of the triumphant Soviet forces - was a main hotspot of the Civil War. Part of the Don Cossacks voisko, dissolved as an administrative unit in 1920, the settlements located westward of Tsaritsyn were a prime target for Soviet authorities. The siege against Cossack communities was also directed at religious life, acknowledged by the regime as an essential component of their identity. The destruction of their churches aimed at uprooting the Cossack traditions, tightly interwoven with Orthodox religious rituals, as well as to eradicate any documentary proofs and historical manuscripts (Olson, 2004; Skinner, 1994). In line with the broader forced secularisation agenda led against church properties and religious groups (Froese, 2004), these actions had a dramatic impact on the life of most Cossack communities. Sidorov (2000:222) distinguishes between several waves of church closures that unfolded between the Bolshevik Revolution and the beginning of the Second World War, but points also to the period of Khrushchev's mandate (195866), when around $40 \%$ of the remaining churches were destroyed. According to figures provided by the local diocese, in the Volgograd (formerly Stalingrad) oblast, from over 600 churches registered before the Bolshevik Revolution, only 32 survived by the fall of the Soviet Union in 1991.

Following the Perestroika reforms initiated in the mid-eighties and the collapse of the Soviet Union, attempts to restore religious life and the status of the Russian Orthodox Church gained some momentum (Davis, 2003; Della Dora and Sooväli-Sepping, 2009). This was also the case in the Volgograd oblast, where upon the formal establishment of the Volgograd diocese in 1991, the few remaining churches were reopened. Nevertheless, after losing $95 \%$ of all religious edifices the diocese faced a rather grim perspective. While providing the religious service to the faithful living in cities was still possible, most of the villages without churches were scattered across the oblast and seemed far out of reach. In an interview from the mid-nineties, one of villagers from the Cossack settlement of Kachalinskaya evoked the central role previously held by the church in their community by declaring: "The steppe and the desert that are here have not only physical but also a moral and spiritual significance, that is the desolation following the power of the communists, the communist regime. There is a complete spiritual desert here (...) Why is this so? Because there is no God here; the people were deprived of faith."'12

As part of the attempts to revive faith practices in the oblast, some clergy members assisted by a group of outdoor enthusiasts begun organising missionary trips in order to provide religious services to rural communities located along the Don and Volga rivers. The teams assembled for these early missions pioneered the revival of

${ }^{12}$ Interview in the documentary $A$ Ship of God: https://www.youtube.com/watch?v=ZvPkZAjF5Ow 
Orthodox faith in Volgograd's rural areas by travelling around in canoes with icons and sacred relics. In an interesting twist, the once central role of the church was now being reworked in the margins through encounters marked by a rather spontaneous mixture of religious services with bard music performances and storytelling. These early missions reached also the Don River areas, known for their remaining Cossack settlements. Here, as in many other places in Russia, during the early nineties the revival of religious practices was spurred by the reaffirmation of the Cossacks lore and identity (Olson, 2004). Although these trips were very successful, they could only cover a limited part of the oblast territory; however, the situation would soon change.

IV.3.3. Saint Nicholas return: the repair vessel, the barge and the landing craft

During the mid-nineties Vladimir Koretsky - the organiser of the Volgograd festival of bard songs and a main figure currently associated with the floating churches - took a memorial journey as homage to Vladimir Vysotsky's oeuvre. Destination was set to Toronto, where Vysotsky previously performed and was supposed to return for another concert. However, his sudden death in 1980 (aged 42) prevented him from keeping the commitment. Being a major admirer and performer of Vysotsky's repertoire, Vladimir planned to organise a tribute concert where his favourite artist never got the chance to return and meet his fans. Thus, in 1996 Vladimir and his older son embarked on the temerarious endeavour of sailing to Toronto in a tiny yacht, which seemed to be anything but a vessel fitted to travel from the Azov Sea to the East Coast. After a three-month adventure at sea, with their lives often being at stake, they managed to reach Toronto and organise the Vysotsky tribute concert. Apparently, a key moment of their journey was the passage between Gibraltar and Madeira, when they were caught in a big storm that almost wrecked their yacht. According to Vladimir, while tied to the boat and struggling to stay alive he had a revelation and promised his son to build a floating church if they would make it safe back home. Upon their arrival in Volgograd, Vladimir kept his promise and, somehow mirroring the story of Yankov, he asked the local diocese to support the construction of a floating church meant to serve the rural areas in the oblast. The idea was welcomed by the metropolitan and local clergymen, yet the diocese was lacking the means to finance such a project. However, less than a year later, a Dutch Orthodox priest representing the Aid to the Church in Need (ACN) organisation visited Volgograd and the yacht club Parus, where he learned about Vladimir's plan. Enthusiastic about the idea, he embraced the cause and quickly managed to secure some donations to initiate the project. Around the same time, in Novosibirsk, a new initiative was becoming increasingly popular. Established with the concourse of priests, medics, social workers and artists, the charity ship Andrei Pervozvannyi travelled along the $\mathrm{Ob}$ River to provide assistance to all communities within reach (Ivanov and Suprun, 
2006). Inspired by the story of Saint Nicholas steamer and the charity mission in Novosibirsk, the team assembled for the realization of Volgograd's first floating church.

With limited resources available, the preliminary discussions focused on the viability of different options to build the floating church and on the area where this would operate. The Cossack villages along the Don River - most affected by the Soviet oppressive spree - were rather isolated and could easily be reached by water; it was thus decided that the church would travel to those parts of the oblast. Contrary to initial expectations, the search for an affordable ship to accommodate the religious function was relatively short. With support from the management of a shipyard in Volgograd, the team obtained an old repair vessel used to provide technical assistance to ships transiting the oblast. Despite its poor condition, the vessel had some reasonably-sized cabins, a kitchen unit and, most importantly, as comically pointed out by one of the priests, "it was still floating" (interview). Compared to the elaborated conversion of the wheel steamer in Astrakhan, the Volgograd floating church had a fairly simple design:

"It was quite primitive. The main role was...we have a church, it will visit the villages, celebrate the Liturgy, baptise people, there will be marriages, sometimes funerals and that's it, because the ship will be there only for two or three days (...) And then it will move to another village, let's say ten kilometres away" (interview).

Since it was not equipped with an engine, a tugboat was needed to tow it around. During the conversion, two of the larger cabins were merged to create church space (figure 4), while the remaining ones accommodated the small crew. In addition to the hull restoration and painting works, the vessel received a central drum ending in an onion dome, which brought it closer to the appearance of a regular church (figure 5). After some deliberation, the team involved in its construction and the diocese decided the floating church to be named Saint Innocent, celebrated in Eastern Orthodoxy for his missionary work. On May 22 $2^{\text {nd }} 1998$, Saint Innocent was consecrated by the central river promenade in Volgograd, receiving the blessing of metropolitan German. A couple of weeks later it took its maiden voyage, setting course to the Volga-Don canal and the Don River.

Accounts provided by participants to its missionary trips reveal that the initial encounters with the floating church were rather humorous. In Nariman, one of the first villages reached by Saint Innocent, people were very surprised when the church tower appeared from between reeds and hearing its bells ringing, some thought they were hallucinating ${ }^{13}$. According to the diocese spokesperson, initially many people found the floating church strange and assumed it belonged to a sect. However,

\footnotetext{
${ }^{13}$ Account evoked by several informants.
} 
following its initial voyage, Saint Innocent soon became a familiar sight in the western steppes of the oblast, mooring nearby each village for around three days. By the end of its inaugural season, it grew extremely popular among the visited villages and the faithful started to appropriate it as their own church. During the first year the floating church 'visited 28 villages, where 446 people were baptised, 1500 believers received communion, 2700 took part in corporate prayers' (Ivanov and Suprun, 2006:142).

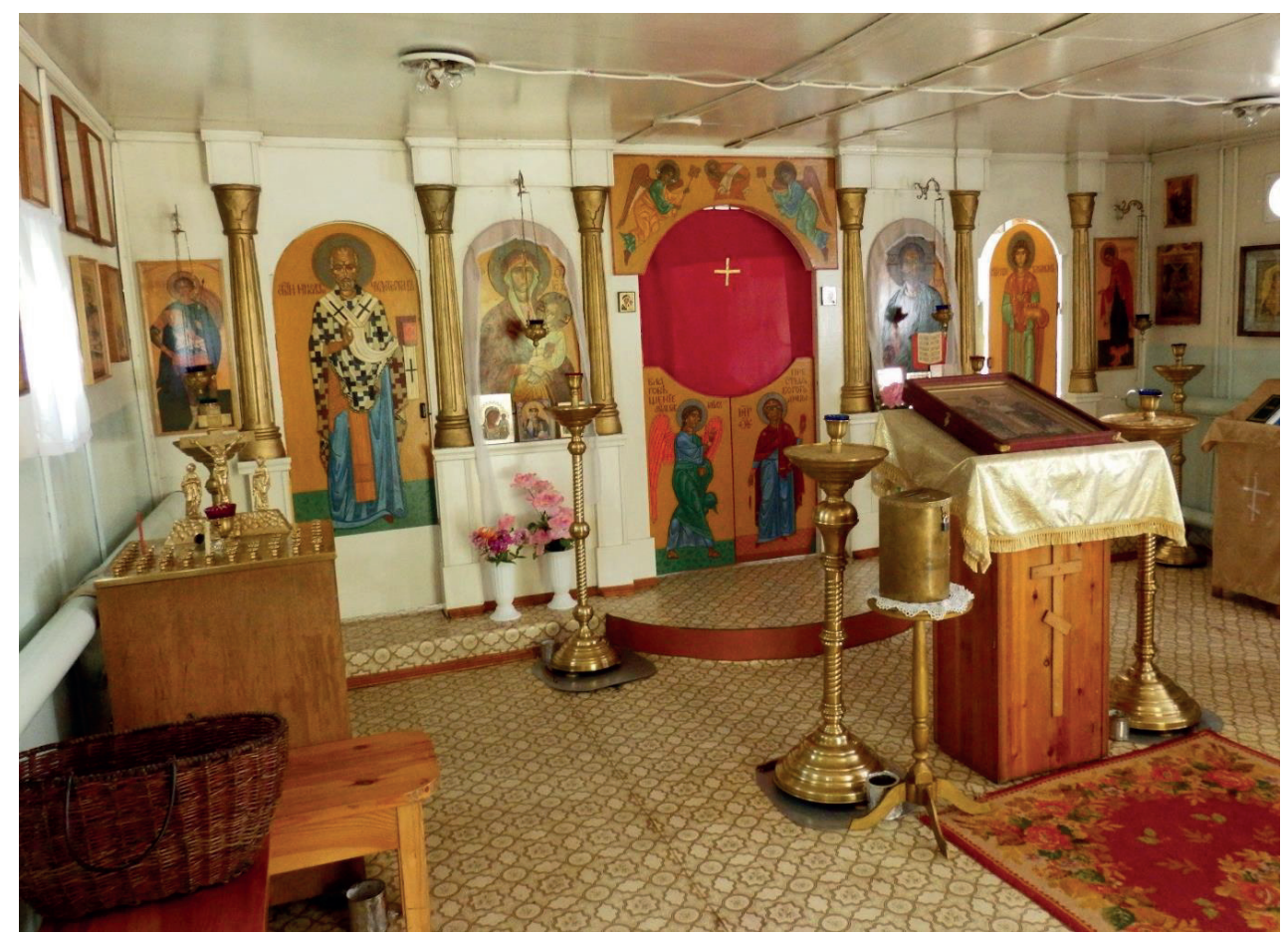

Figure 4. View of Saint Innocent iconostasis and nave. Source: The Authors

Although travelling around for only three to four months per year it covered a significant area, from the Volga-Don canal up to the town of Logovskiy, near the border with Rostov oblast. For the remaining time, Saint Innocent was stationed halfway in the village of Pyatimorsk, so that as many people as possible could still attend the religious service. As pointed out by the crew members and clergymen involved in organising the missionary voyages, serving on the floating church proved to be a great challenge. The priests were faced with a totally different setting compared to that of regular orthodox parishes, since they constantly had to adapt to the changing conditions and shifting spatialities of Volgograd riverscapes:

"An ordinary church is fixed in one place and you get used to a certain order of things, while on the floating church you don't know when the other ship will tie-up to you. You don't know how people will react to the presence of the floating church and you have to 
explain them how things are done on the floating church. It's a kind of extreme experience" (interview).

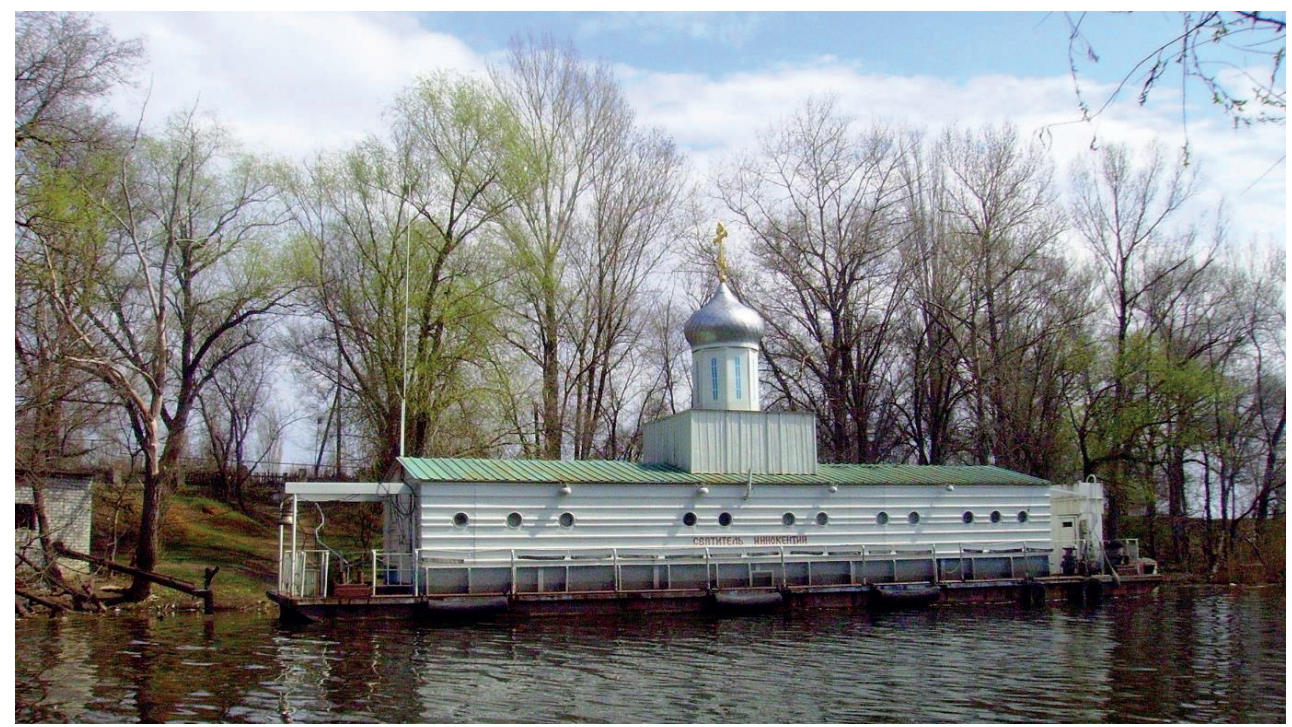

Figure 5. Saint Innocent floating church by one of the mooring sites. Source: Sergey Ivanov

A messenger appointed by the diocese used to travel around the Don area, announcing the arrival of the floating church in advance. Apart from gathering to celebrate the Liturgy, people could schedule baptisms and marriages or ask the priest to hold a rain prayer service during periods of drought. As a distinct feature of religious ritual, many baptisms were taking place in the Don waters, revealing the deep connection between faith and the river in the life of those communities. Saint Innocent's missionary journeys had an outstanding impact on the revival of religious practices on the Don River. According to the Volgograd diocese, after five years the floating church entered service, half of the villages it used to visit established their own parishes.

The mobile-shared character of Saint Innocent has arguably enabled the displacement of the 'absent' religious centres of visited communities to mooring sites, articulating a topology of fixed points and shifting spatialities. As such, mooring sites emerged as temporary centres of spiritual encounter through which affects and materialities were being recast. Appropriated as both singular and multiple in substituting for previously destroyed churches, Saint Innocent warped its operative area by overriding the marginal condition of the villages sharing its service. As a consequence of Saint Innocent's initial impact, the ACN organisation decided to provide support for the construction of a second floating church in Volgograd (figure 6). 

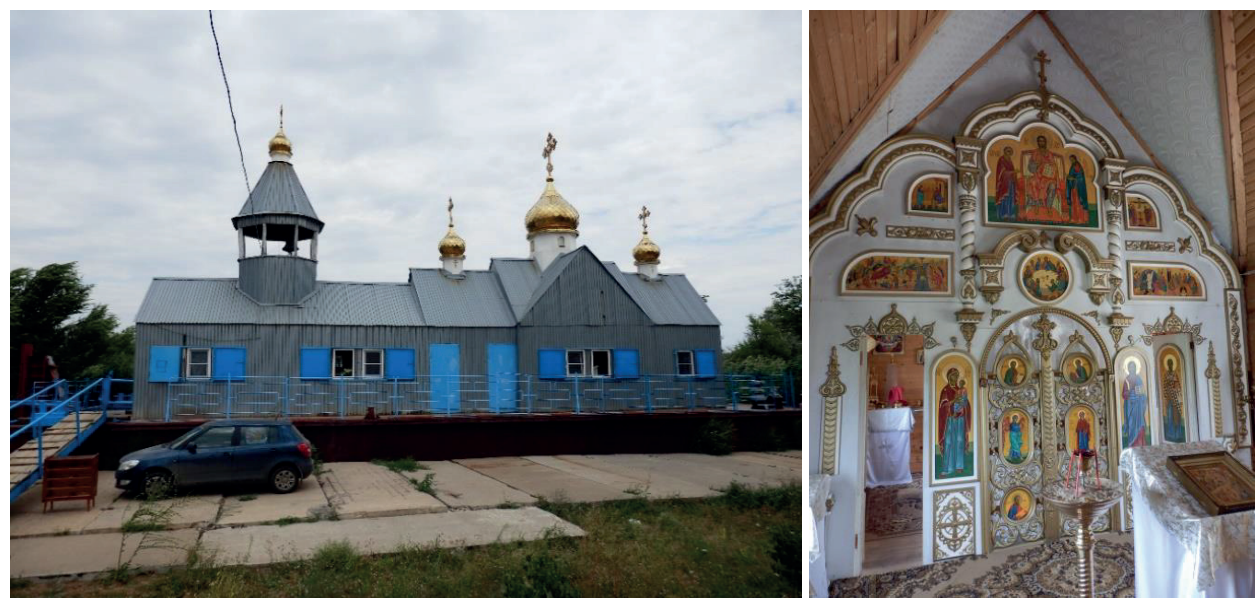

Figure 6. View of Saint Nicholas stationed in Oktyabrsky village (left) and its iconostasis (right); Source: The Authors

Built on top of an old barge, it was slightly larger than Saint Innocent and had a layout very similar to most common Orthodox churches. With the sustained effort of many volunteers, a couple of years after Saint Innocent took its first voyage, a new floating church named Saint Nicholas was consecrated on the Volga. At first, Saint Nicholas was moored at the yacht club Dinamo in Volgograd, where it served as a place of worship for people living in the surroundings and the ship crews navigating on the Volga. A few years later, it was towed to Oktyabrsky village to be used as a stationary church by people living in the southern parts of the oblast.

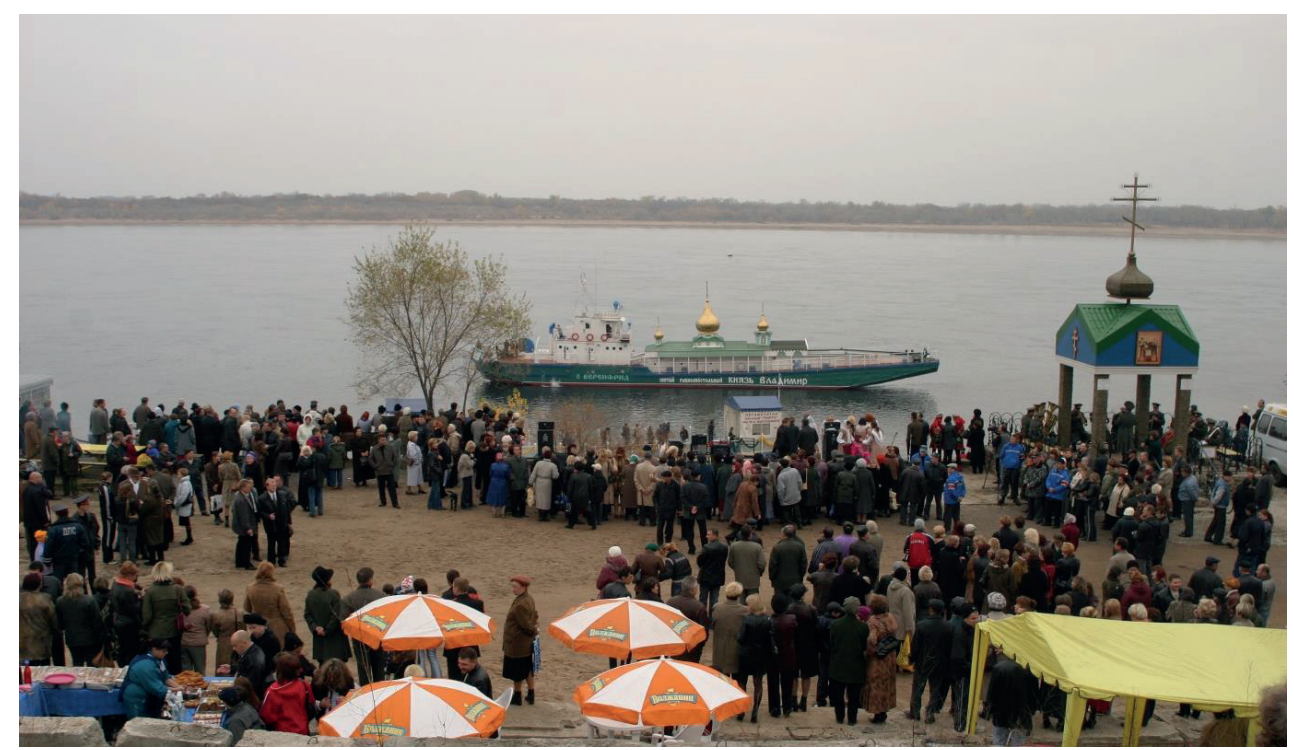

Figure 7. The consecration of Saint Vladimir by the Volga embankment. Source: Sergey Ivanov 
In the meanwhile at the yacht club Parus, where this all started, Vladimir was planning an even more ambitious project. After the enormous success the first two floating churches had in the Volgograd oblast, he envisioned a more reliable self-propelled one, capable of travelling long distances. The plan was to build a floating church that would serve all the communities within its reach along the Volga, from the Caspian Sea to Moscow. With the approval of Volgograd diocese, in 2002 Vladimir started looking for a shallow-draft vessel to be turned into a floating church. In the same year, he managed to obtain an old decommissioned landing craft from a shipyard close to Saint Petersburg. The resources required for the project were secured through various donations and the support of ACN. The complex repair works and the conversion of the ship took almost two years, yet the result was a spectacular one. At the end of October 2004, it was consecrated by the Volga embankment, where the festival of bard songs is held every year (figure 7). The flagship of the 'flotilla for God' was named after the ACN founder, father Werenfried van Straaten, while the church on board was named after Saint Vladimir. During its maiden voyage it reached Saratov to the north and Astrakhan to the south, stopping in every village and town to provide religious services. While mooring in major cities, once the Liturgy was celebrated aboard, Vladimir used to hold charity recitals by performing Vysotsky's repertoire. In an interesting twist, almost a century after Saint Nicholas steamer took its first Volga journey, a new floating church was visiting Astrakhan, carrying sacred relics and the music of one of Russia's most beloved bards.

\section{IV.4. Conclusion}

At the outset, we introduced our case study by referring to the annual festival of bard songs by the Volga River embankment. Partly floating and partly accommodated on ground, with its experimental setup and eclectic mix of performances, the festival mirrors in many regards the complex narratives and trajectories that have informed the construction of the floating churches. The early missions jointly established by volunteers and Volgograd diocese, the revival of Cossacks traditions, the folk songs and artworks celebrating everyday life by the river, would later reflect in the form of religious rituals and the way the floating churches were built. In line with Peter Brook's (1996:45) account on theatrical performance and religious ritual, here it was "the ceremony in all its meanings" that "dictated the shape of the place". And the resulting place was mobile too, as mobile as it can get. Exploring the movement of holy sites "through human and nonhuman bodies" Della Dora (2011:169-170) argues that places travel "beyond their physical boundaries, through the intricate circuits of society and culture". Added to the fluid character of holy sites as enacted by different forms of spiritual encounter - from pilgrimages to circuits of religious memorabilia the mobility of the floating churches distinguishes through a range of temporal and 
functional displacements that triggered both the reworking and the stabilisation of their operative areas. Enhanced by the shifting spatialities of religious rituals and Volgograd's riverscapes, their dynamic sway has fostered new envelopments of materialities and affects that have reanimated the sediments of a contested order. Consequently, the floating churches acted as alternative archives in producing effective counter-narratives to the void created by the oppressive campaigns previously directed against religious life. As mobile sacred spaces, they eroded the formerly marginal status of the communities within their reach. We have seen this at work via their role in patching up a constellation of sites completely stripped of religious infrastructure, which culminated with the establishment of parishes in many of the places they visited. Through their mobile-experimental character "spatialities are folded together and fractured; their past, present and futures entangled with the changing relations" (Davies, 2010:668). The floating churches have thus enabled alternative conditions of negotiation and attunement between different practices, by weaving a whole range of absences and presences into topological arrangements that contracted and expanded with the shifting spatialities of religious rituals and the meanings attached to mooring sites. In turn, this has led to the emergence of new recursive functions and fixed points that "nearly always come about through involved experimentation rather than deliberative thinking" (Thrift 2008:123).

The experimental character of the floating churches is also reflected in their hybrid and rudimentary appearance, as an expression of creative reuse beyond the immediate affordances dictated by circumstances of disposal or dissolution. Resulted from the cross-breeding of a panoply of things with various 'affective qualities' (Anderson, 2009), the floating churches exhibit a wide spectrum of resonance articulated by both their inscribed and unanticipated functions. They are in themselves spatiotemporal ecologies of things - each with their own temporality and role in 'timing life' (Crang, 2012:2121) - open to various couplings between human and non-buman bodies. Mimicking the intricate river topologies they navigate upon, their voyages have become tributaries to new imaginative geographies of affects, from their connection to various creative manifestations and the ingenious staging of religious rituals to absences that are not yet manifest. As previously pointed out, they mirror past developments and historical practices through "temporary stabilizations of things and relations, as coming into being and as coming apart" (Crang, 2010:1085), be they abandoned ships, sacred relics or folk repertoires. Thus, the ways in which the floating churches mobilised this shared surplus is what arguably endorses them as an innovative response to processes of identity formation and place-making in Volgograd oblast. In following Cresswell's (2012:175) call for a more generous reading of archiving practices, the case of the floating churches arguably exposes "the utility of thinking of other kinds of collecting and other kinds of space as archival, including places themselves". Through the 
creative assembly of their materialities and reinterpretation of their functional registers, the floating churches emerge as mobile cosmologies/theologies that, beyond reworking the connections between past and present, also entertain the perspective of a betteryet-to-come. By animating different imaginaries and views on the transcendent, they act like rudimentary warp bubbles that stretch and bend the spatiotemporal continuum within their reach. However, this is not the kind of innovation normally celebrated in the powerhouses of creative destruction; rather it originates in the interstices of "the presumably religious and the secular modern" (Tse, 2014:214), where multidimensional hybrids, as the ones we have just explored, surface through creative endeavours enthused by the "passionate imitation that derives from religious ritual and still partakes of its spirit" (Girard, 1990:19). 


\section{V.A harbour on land: De Ceuvel's amphibious topologies of creative reuse*}

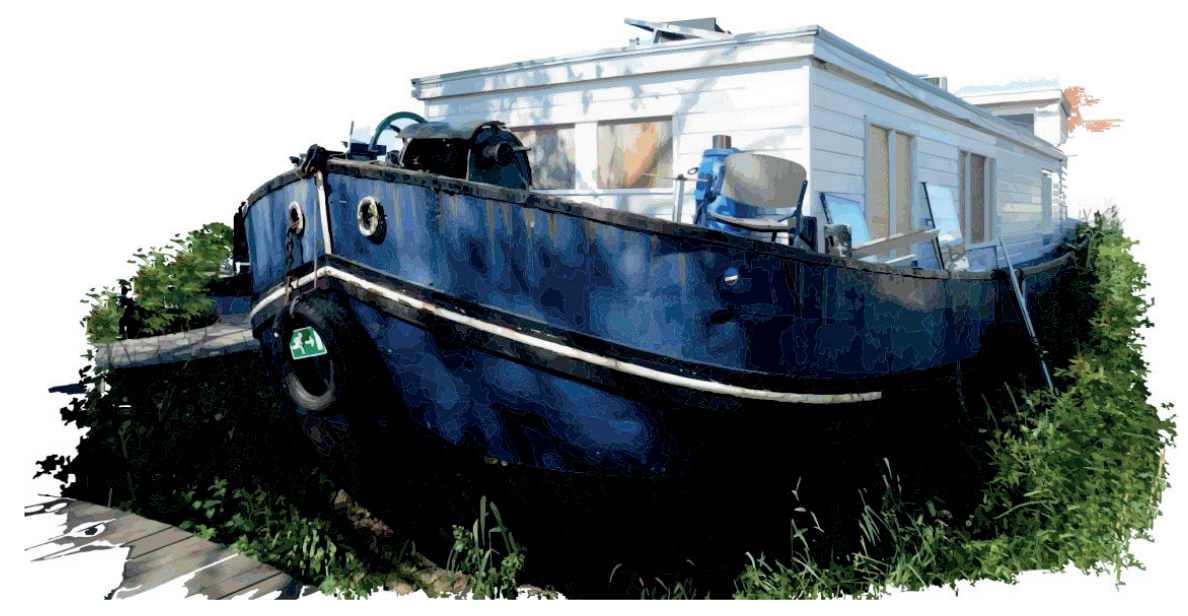

*This chapter is based on the version submitted to Environment and Planning A:

Barba Lata I and Duineveld M (under review) A harbour on land: De Ceuvel's amphibious topologies of creative reuse. Environment and Planning $A$. 


\title{
V. A harbour on land: De Ceuvel's amphibious topologies of creative reuse
}

\begin{abstract}
This article examines the role of creative reuse as an alternative imaginary specifically concerned with the residual surplus that results along dominant processes of accumulation and value production. In moving beyond circumstances of disposal or dissolution, we argue that creative reuse interventions provide inventive ways to exploit the productive latencies scored across incumbent sociotechnical arrangements. Building upon an ethnographic study of De Ceuvel's on-land harbour, an experimental self-sufficient community recently established in Amsterdam, we show how things that were otherwise redundant/disposed/forgotten can stimulate new material and affective dispositions that call into question established practices around sustainable, creative and inclusive city-making. Based on the findings, we go on to suggest that creative reuse interventions enable new conditions of possibility for the enactment of alternative urban futures.
\end{abstract}

Keywords: on-land harbour, creative reuse, topology, materiality, innovation, Amsterdam

"I am enthusiastic over humanity's extraordinary and sometimes very timely ingenuities. If you are in a shipwreck and all the boats are gone, a piano top buoyant enough to keep you afloat that comes along makes a fortuitous life preserver. But this is not to say that the best way to design a life preserver is in the form of a piano top. I think that we are clinging to a great many piano tops in accepting yesterday's fortuitous contrivings as constituting the only means for solving a given problem.” (Fuller, 1969:9)

\section{V.1. Introduction}

Crossing the River IJ by ferry from Amsterdam Centraal it takes roughly ten minutes to reach Buiksloterham, a patchwork of docklands and industrial sites stretching for several kilometres along the northern riverfront. Once a main powerhouse of the Dutch shipbuilding industries, the area has turned over the past decade from a desolate industrial landscape into a cultural hotspot and one of Amsterdam's hippest places. With the support of municipal authorities, several parts of Buiksloterham have 
become breeding places for self-organising communities, actively engaged in the regeneration of IJ's northern riverfront. Among the recently established ones we find De Ceuvel, located on the premises of former De Ceuvel-Volharding shipyard. A narrow street bordered by old workshops provides access to the area and upon arrival you are struck by an odd arrangement, more likely to be found on the canal waters nearby rather than on land. Its post-industrial and somewhat precarious appearance has almost a surreal feel to it. Stranded houseboats connected by a winding boardwalk (figure 8) and a café-restaurant built on harbour wooden posts, all surrounded by lush greenery. The set-up is completed by a terrace with rough wooden tables, benches made of old rowing boats, a jetty and a floating garden located at the end of the shipyard's rusty slipway. Despite its unconventional look, De Ceuvel is home to a vibrant creative community and a cleantech playground that merge in the ambition of becoming one of "the most unique and sustainable urban developments in Europe"14.

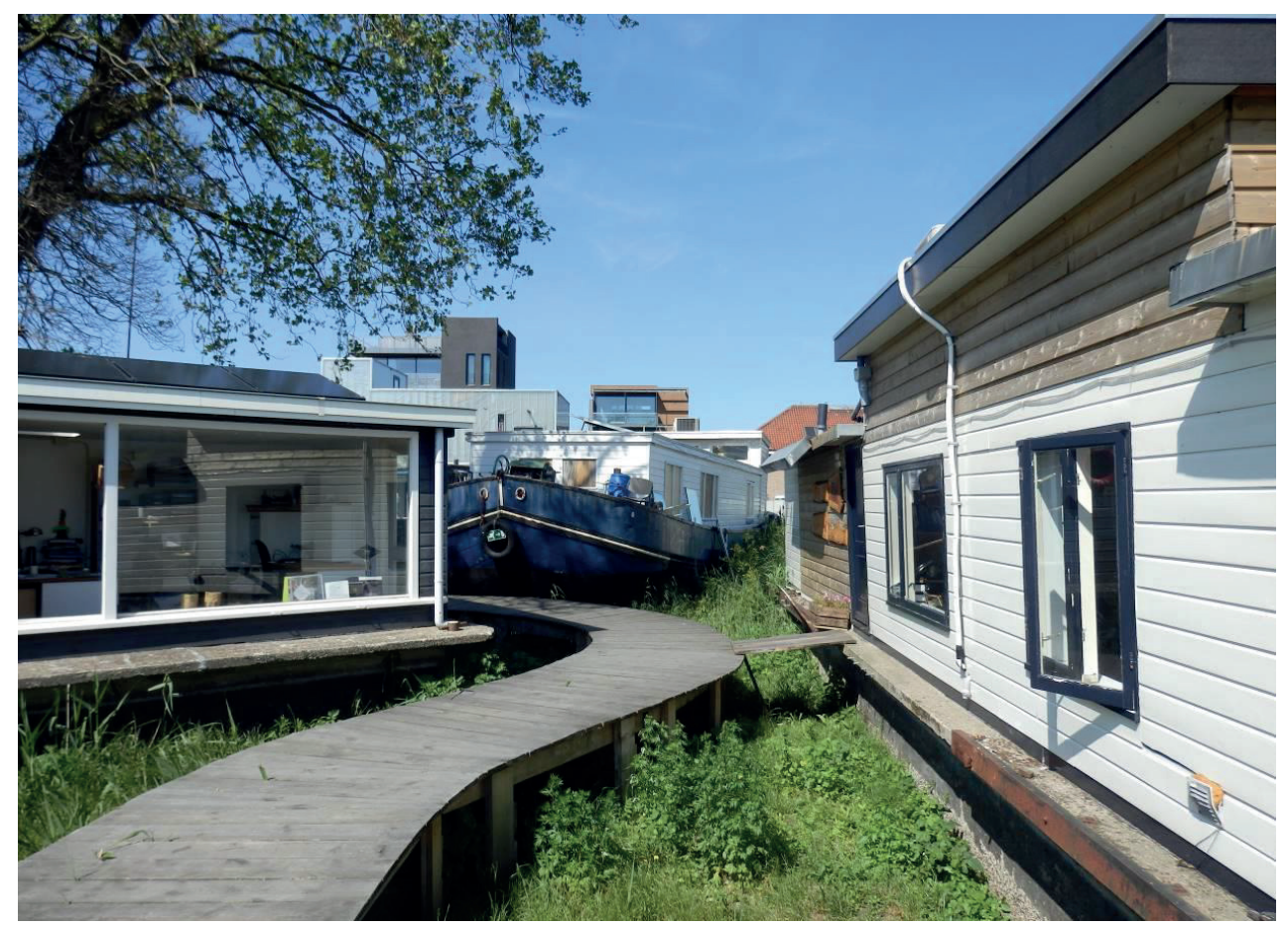

Figure 8. Upcycled houseboats at De Cenvel. Source: The authors

In 2012, the on-land harbour concept coupled to a phytoremediation park project has won the competition organised by Amsterdam municipality for a ten-year lease of the industrial site. Later additions to the initial plan included a commercial function and designated public space, as well as an integrated system for energy, water and food

14 De Ceuvel's mission statement: http://deceuvel.nl/en/about/general-information/ 
production. After the official opening in June 2014, De Ceuvel has rapidly turned into a key exponent of sustainable urban living in Amsterdam Noord, and currently scores as one of the most successful initiatives of its kind in the Netherlands. Notwithstanding the initial planning and vision, the project implementation moved forward in fits and starts. Financial and logistical constraints, bending the building codes and bypassing urban regulations to allow for the use of experimental and often low-tech solutions, added to the pressure of tight deadlines proved anything but a smooth jaunt for the community pioneers. However, even from an early stage the project grew extremely popular and brought together an impressive pool of volunteers, as well as a motley crew of creative professionals that used the stranded houseboats to create their own customised workplaces. Theatre artists, filmmakers, 'ecopreneurs', architects and designers adhered to De Ceuvel's promise, while coupling it to their own agendas in an innovative and largely improvised collaborative arrangement. Through its makeshift character, De Ceuvel emerges as a place "of inspiration incorporating many possible worlds" (Thrift, 2006:291) that enable the production of alternative value regimes and urban materialities. Animated from its very inception by a logic of "flexible, cheap and off the grid"15, the on-land harbour distinguishes as a landmark of creative reuse, which both subsumes and exceeds the residual materialities informing its making.

Exploring De Ceuvel's liminality and ethos of creative reuse involved an ethnographic study focused on the actor coalitions and material displacements that allowed this experimental initiative to take off. The study has included twenty semi-structured interviews with community members, policymakers, planners and researchers. Additionally, we have made use of participant observation techniques, informal talks with visitors and local residents, as well as an extensive survey of research reports, media articles and archive materials. Based on the findings, the argument developed in this article is threefold. First, we explore the role of creative reuse imaginaries in providing alternative ways to expose and alter the productive latencies which result along dominant processes of accumulation and value production. Second, we suggest that the explored instances of creative reuse transcend circumstances of disposal or dissolution and, as such, they enable the conditions for things that were otherwise redundant/disposed/forgotten to make surprising returns and morph into new material and affective dispositions that call into question established norms and practices. Third, we provide a topological reading of how alternatives of creative reuse could be understood as part of the broader interplay of multiple urban imaginaries that alter both the status of things and processes of place-making. In substantiating the three main lines of argument, the next section brings into dialogue the corpus of enquiry on governance

${ }^{15}$ Description of the initial plan, by one of the partners from Space \& Matter architecture studio: http://www.smart-magazine.com/en/de-ceuvel-amsterdam-urban-project/ 
experiments and grassroots innovations with the strands of work on materiality, mobility and affect. The empirical section that follows, provides an in-depth discussion of creative reuse as a multidimensional imaginary feeding into alternative urban materialities and sociotechnical innovations that are politically Other to, yet inseparable from, the realities of incumbent formal domains. The discussion section and conclusion elaborate further on the topological sway of creative reuse interventions in creating new conditions of possibility for the enactment of alternative urban futures.

\section{V.2. Alternatives of creative reuse: reanimating urban materialities}

"Not architecture alone but all technology is, at certain stages, evidence of a collective dream." (Benjamin, 2002:152)

Creative reuse owes its conceptual substrate to several strands of thought committed, for instance, to the historicity of innovation narratives (Benjamin, 2002; Tarde, 1903), notions of materiality and affect (Anderson and Wylie, 2009), or the study of transformative practices and related forms of experiment (Davies, 2010). To deepen the understanding of the case study presented in this article, we connect some of these ideas to the question of alternatives and their logic of displacement. To do so, the first part of our conceptual exploration draws on the recent spate of attempts to reimagine urban sociality, from grassroots innovations (Simone, 2008) and progressive coalitions (McFarlane, 2012), to the new infrastructural turn (Amin, 2014) and the burgeoning corpus on smart urbanism (Greenfield, 2013). Within this framework, creative reuse is proposed as an alternative imaginary informing innovative responses that are politically Other to, yet inseparable from, "the realities of creative destruction and accumulation by dispossession" (Vasudevan, 2015b:349, emphasis added). As such, it operates as the referent of interventions that carve out the productive latencies of incumbent sociotechnical arrangements. Along this line of reasoning, the second part of our conceptual exploration is specifically concerned with the dynamics of creative reuse in exposing and altering the residual surplus of various materialities and affects that city-making processes rest upon. Lastly, we dwell on the role of topological thinking as a resourceful modality to interrogate the material and affective dispositions that lend to makeshift sociotechnical arrangements and emergent alternatives.

\section{V.2.1 Alternative conditions of possibility}

The Oxford dictionary ${ }^{16}$ provides two main definitions of the word alternative. The first pertains to "one or more things available as another possibility or choice" and the second, "to activities that depart from or challenge traditional norms". Both imports

16 Oxford Dictionaries online: http://www.oxforddictionaries.com/definition/english/alternative 
feature a role in our conceptual enterprise. The first, as a matter of choice and causality, would bring us closer to Anderson et al.'s (2012:186) account on the purposive cut "within assemblages", meaning that "we might strategically choose who or what to hold to account as an ethical or political stance". This perspective concerns the mongrel character of alternatives and their inherently multiple political incarnations. Accordingly, the second import is suggestive of the dislocation work prompted by the emergence of alternatives in relation to dominant imaginaries. With hindsight to the past decade or so, both meanings appear as instrumental to a broad range of scholarly dispositions cultivated toward 'marginal' and informal articulations of urban everyday practices. If for some alternatives flag the struggle for recognition and, often, survival (Ghertner, 2008; Pickerill and Chatterton, 2006; Soja, 2009), others call attention to makeshift coalitions and governance experiments which transcend the logic of institutional arrangements and their regulatory frameworks (Brown et al., 2012; Bulkeley and Castán Broto, 2013; Richardson et al., 2014). By and large, what many of these instances arguably share is an onus on envisioning "more hopeful urban futures" (Purcell, 2008:34). As AbdouMaliq Simone (2008:30) contends, while animated by the swirl of everyday life, the city acts as "a constant reminder of what could be but [still] isn't”.

Over the past years, the above line of reasoning was seminal to a more generous reading of urban politics in both the Global South and North, where acts of resistance and contestation, as much as the learning processes enabled by them, are in fact increasingly highlighted as catalytic to new sociotechnical synergies and alternative innovations (McFarlane, 2011a; Roy, 2009). These include, yet are not limited to, the treatment of radical self-sufficient communities (Pickerill and Maxey, 2009; Van Assche et al., 2013), precarious infrastructures (Amin, 2015; Morales et al., 2014) and grassroots solutions for sustainable development (Davies and Mullin, 2012; Seyfang and Smith, 2007), or of governance arenas (Bulkeley et al., 2014; Evans, 2011) and smart urbanism visions more broadly (Calzada and Cobo, 2015; Luque et al., 2014). For Van Assche et al. (2013:239), informal communities operating in contexts that elude the immediate reach of incumbent regimes, rely on fluid governance arrangements to which "flexibility in role distribution is an enabling factor" for social learning and innovation. In a similar vein, Jensen et al. (2015:566) discuss the role of harbour swimming in Copenhagen "as a transformative urban practice" occasioned by "navigational actions" across multiple enactments of the harbour area. The fluid/ navigational metaphor distinguishes as particularly relevant here, on one hand, "in recognition of how change often is moulded by loosely coordinated micropolitical manoeuvres of actors and actor constellations operating in the absence of predefined strategic visions" (Jensen et al., 2015:566) and, on the other, in expressing the myriad 
of urban imaginaries constantly recast through the interplay between formal and informal agendas.

In addressing the interplay between formal and informal practices in cities, McFarlane and Vasudevan (2014:257) point to "the below-the-radar practices of everyday life and culture that not only grease the wheels of these apparently formal realms, but which may indeed be more important than the formal domain". While animated by an ethos of improvisation and experimentation that seeks to attain social impact with often precarious means (MacLeod and Jones, 2011), the ingenuity of such alternatives plays most of the time second fiddle to the cohabitation with their 'formal' referents, through shifting "entanglements between objects and bodies, discourses and power, performances and blueprints for actions" (Lancione, 2016:13). This aspect invites to a more careful reflection on the status of 'informally-sourced' alternatives from a broader outlook, beyond idealising their condition as marginal, rudimental or otherwise (Luque-Ayala and Marvin, 2015; Sundaram, 2010), by considering the dynamic qualities of their material and affective dispositions in rerouting narratives of belonging. As Amin (2014:157-158) suggests, instead of romanticizing "occupation and informality as a new dawn", attention should be directed at how "the right of the disenfranchised to claim the city is exercised and shown to be possible". Related to this last point, and as shown by many thus far, creativity is as much a collective resource as it is an essential feature of any practice, a view which has inspired some potent counter-narratives in usurping the normative assumptions on creative elites, creative cities, and the like (Boyle, 2011; Jeanes, 2006; Peck, 2005).

\section{V.2.2 Reanimating urban materialities}

Bottom line, this recent spate of interventions delivers a clear message that cities muster an outstanding creative potential still poorly acknowledged, where notions of creativity, entrepreneurship or innovation afford as many connotations as the daily 'routines' of struggle for recognition and a better-yet-to-come (Amin, 2014; McFarlane, 2012; Simone, 2010; Vasudevan, 2015a). The making of urban future(s) is a function of these multiple and contingent imaginaries, which unveil instances of an 'anticipatory politics' (MacLeod and Jones, 2011:2449) that is inherently transient, transgressive, and tactical rather than strategic, as de Certeau (1984:52) would have it. Once approached in this manner, the idea of alternatives comes closer to Appadurai's (2013:295) plea for an 'ethics of possibility', indicative of "those ways of thinking, feeling and acting that increase the horizon of hope, that expand the field of the imagination, that produce greater equity (...) and that widen the field of informed, creative, and critical citizenship". Granted this vantage point, we now turn to sketching how the above treatment of alternatives informs the creative reuse imaginary evoked at the outset, in coupling various material and affective dispositions to city- 
making processes. In its somewhat banal expression, creative reuse intimates an ethos of improvisation, and of surplus, albeit on a different tone than the putative grand narratives on creativity and innovation. There is nothing glorious about it as long as the immediate affordances of 'novelty' or 'change' cling on to the mantras of creative destruction. Whether one takes an interest in informal settlements or the presumably high-end inflections of smart urbanism agendas, creative reuse concerns the residual surplus that results along processes of accumulation and value production.

While inevitably generous in breadth, creative reuse could be regarded as primarily pivoting on those action repertoires that expose and alter the material and affective latencies adjacent to dominant sociotechnical arrangements. Here, adjacency "may or may not imply contact but always implies absence of anything of the same kind in between" (Rabinow, 2008:33). Accordingly, creative reuse pertains to hybridisation processes that scavenge and rework urban materialities through synergies that constantly dog their multiple, dynamic and, occasionally, odd condition (Kwinter, 2007; Thrift, 2008). As Latham and McCormack (2004:719) point out, the "urbanness of cities is precisely a product of this excessive plurality, the multiple attempts to give this plurality shape and form, and the constant exceeding of these limits produced by the city's plurality". Among these multiple attempts, "practices of gathering, composition, alignment, and reuse" (McFarlane, 2011b:649) enable the conditions for synergies and modes of (re)ordering to which "symmetry is always a moving target" (Law, 2004:173, emphasis added). Understood in these terms, creative reuse spans a host of transient interstitial spaces of potentiality and action, where the residual surplus of dominant accumulation sparks off new mixtures and alternative creative manifestations that, in turn, lead to shifting instances of core and periphery. As such, creative reuse distinguishes as symptomatic to the substance injected into particular sociotechnical arrangements to reanimate the sediments of seemingly established orders. Conversely, once this dynamics of aspiration and difference is granted recognition, dichotomising the realities of creative reuse and creative destruction would prove of little use.

If capitalism relies indeed on what Anna Tsing (2015:63) calls 'salvage accumulation', i.e. "taking advantage of value produced without capitalist control", then creative reuse becomes a tactic of the interstitial (not to be mistaken with the marginal), as politically Other to, yet inseparable from, processes of creative destruction. This seems a rather twisted symbiosis, one of peculiar association. However, it is through these odd couplings that the "room for building a politics to confront and navigate salvage accumulation" (Tsing, 2015:65) is made possible. While the question of any viable alternative beyond the reach of an otherwise performative economy still remains (Callon, 2007), the residual surplus generated within packs a remarkable matrix of potentialities, open to all sorts of synergic effects. This perspective resonates with the 
processual philosophy of Michel Serres (2008:81, emphasis added), and his exploration of contingency via the notion of mixture:

"All right, here is mixture. Confluence, unfurling, occupation of places (...) A medium is abstract, dense, homogeneous, almost stable, concentrated; a mixture fluctuates. The medium belongs to solid geometry, as one used to say; a mixture favours fusion and tends toward the fluid. The medium separates, the mixture mitigates; the medium creates classes and the mixture, hybrids."

Following up on Serres, and to iterate the fluid/navigational metaphor, a processual reading of creative reuse would likely prove lucrative to account on the material and affective displacements that enable various imaginaries to morph in yielding alternative city-making trajectories. Aligned to the body of work on the post-human condition of urban sociality (Hinchliffe and Whatmore, 2006), the creative reuse imaginary expands on the efforts to unveil the manifold enrolments that actively shape the fabric of cities, through often transient and unexpected synergies which seem to defy established norms and practices. These matters have inspired the development of more flexible and inclusive spatial grammars, better equipped for a whole array of "spatiotemporalities that previously might not have even been considered properly geographical” (Latham, 2011:313). Among these, topologicallyinformed approaches have gained considerable analytical purchase over the past years (for an overview see, for instance, Amin, 2007; Martin and Secor, 2013).

\section{V.2.3 Topological imaginaries}

In calibrating the creative reuse imaginary further, we rally to McFarlane's (2015:3) recent intervention which tackles urban density "as a topological problem connecting multiple concerns and spaces in ways that have consequences for other spaces, some planned and some unplanned". For McFarlane (2015:10), density understood in dynamic terms -as a function of 'intensive heterogeneities' produced through "nonlinear combinations of often different processes and things"- opens a broad spectrum of enquiry ranging from informal and precarious infrastructures to digitally-enhanced urbanism. On a similar note, Sanford Kwinter (2007:189) contends that in the case of urban materialities "multiplicity and embeddedness, combined with the critical presence of a 'thick' time, allows for the open-ended interactions of parts -the hybridizations, blendings, and conflicts- that effectively destroy a structure's determinism and that feed its wildness". Rendered as such, the plurality and labile character of urban materialities calls for a broader ambit in the study of city-making processes, to include other forms of accumulation and creative action. This would unveil emergent architectures that overflow expert-led designs, or even solely humancentred aspirations, into a "topological terrain of contingently assembled durations, 
velocities, intensities" (Jacobs and Merriman, 2011:218). Taking heterogeneity as the corollary of fusion, challenges a whole range of normative assumptions on the status of things and narratives of belonging. Through its ethos of improvisation, creative reuse becomes the fief of makeshift architects, of fluid sociotechnical arrangements exuded from interstitial spaces of loose connections. As Vasudevan (2015b:355) points out, to "speak of a makeshift urbanism is therefore to acknowledge the constantly changing role of materials and resources in the making of such spaces".

In topological terms, creative reuse can then be thought of as a dynamic operator that persistently dogs the latencies scored across the liminal domains of urban everyday life. In other words, these liminal domains are the spaces of action and experimentation where the accumulation of challenges and of creative potential enables alternative conditions of possibility. In tune with Simone (2013:243, emphasis added), "[w] hat it is possible for people to do with each other is [then] largely a question of what it is that exists between them, and how this 'between' can be shaped as active points of reference, connection and anchorage". While operating within a complex meshwork shaped by both formal and informal practices, it is the unremitting rehearsal of such alternatives that fosters transient 'subsumption architectures', evoked by Kwinter (2007:187) as the referent of wild, bottom-up systems, which "range and explore and mine their environment, that capitalize on accidental successes, store them, and build upon them". Notions of novelty or inventiveness afford more generous connotations in this context, where the ingenuity of sociotechnical arrangements particularly lies in their dynamics of attunement, rather than their material articulations (McFarlane 2011b:654; see also, Amin 2015). Animated by a logic of aspiration and difference, these situated responses performed across hotspots of informality share an affinity with Deleuze's (1994:162) topological treatment of the problematic, as an expression of multiplicities and which "tends to give rise to discontinuity on the basis of continuity, or to ground solutions in the conditions of the problems".

To return to the point on expert-led designs, the Deleuzian problematic flags the performative and contingent character of alternatives (or alternative solutions), revealing instances of creativity as both a distributed and relational resource. This line of reasoning proves politically salient in avoiding what McCann (2011:124, emphasis added) refers to as the "dangerous tendency toward diffusionism", which "involves a belief that inventiveness is scarce and concentrated in a few advanced and progressive places from which innovations flow to the rest of the world". In thinking about knowledge mobilisation, Faulconbridge (2013:340-341) pleads for a reading of "its spatiality and potentiality (positive and negative) by focusing less on the travels from place to place of a single knowledge practice, and more on the topological intersections of multiple mobilising knowledges that are assembled in any city, something that results in new 
and embedded knowledges emerging". Thus, topological imaginaries provide the means to think beyond the axiomatic dimensions of city-making processes, to witness how their seemingly residual surplus becomes a vivid source for makeshift arrangements actively engaged in sketching alternative future trajectories.

\section{V.3. De Ceuvel: on-land harbour and fluid sociotechnical arrangements}

"Not being able to build there, but then to decide in a place where in the past they used to build ships to put the ships on the ground is quite...it is a terrific idea, it is really an invention" (interview)

Flood protection works and floating technologies score as a longstanding signature of Dutch engineering (Zegwaard and Wester, 2014). Along this legacy of negotiating development at the interface between land and water, the past couple of decades have sparked an intense phase of experimentation with floating urbanization solutions, ranging from technical infrastructures to the establishment of water-based communities. These have delivered some spectacular designs, which showcase their potential to accommodate future urban functions on water, as with the floating neighbourhood of IJburg Amsterdam or the Climate Adaptation Pavilion in Rotterdam. However, an interesting twist in the unfolding of these emergent urban materialities concerns their branching trajectories, through circulation processes between practices and places, with the resulting heterogeneity becoming "a spur for the creative [re] use of mobile knowledges to generate new situated solutions and practices through experimentation and hybridisation"(Faulconbridge, 2013:340). Hence, as imaginative as these floating technologies might be in providing alternatives for more resilient cities, they also afford all sorts of other unexpected inflections. In this section we dwell on such an inflection, which despite its apparent stillness has plenty to share with its floating and mobile referents. Pivoting on creative reuse interventions, the case of De Ceuvel exposes a makeshift sociotechnical arrangement that enabled various residual materialities, including the seemingly stranded houseboats, to pitch and roll under ad hoc actor coalitions and synergies between rudimentary and novel technologies. As such, the making of De Ceuvel's on-land harbour rippled through Amsterdam's urban fabric in some enthralling ways. In what follows, we explore these in relation to the points raised throughout the previous section.

\section{V.3.1 Creative reuse imaginaries and ad hoc coalitions}

At the outset, we evoked De Ceuvel's post-industrial and somewhat precarious appearance as an expression of liminality and improvisation. While certainly distinguishing through its idiosyncratic layout, De Ceuvel also shares a broader 
experimental onus with Amsterdam's established tradition of progressive movements, from the latest interventions in Buiksloterham to the tumultuous history of social centres and urban squatting (Owens, 2009; Soja, 2002). In many regards these are redolent with the dawns of the Situationist International (Nieuwenhuis and Debord, 1958) or with Aldo van Eyck's creative recovery of derelict areas through playgrounds in post-WWII Amsterdam, which heralded an alternative urban agenda based on a "shift from the top down organization of space by modernist functionalist architects, towards a bottom up architecture that literally aimed to give space to the imagination" (Oudenampsen, 2010:25; Lefaivre, 2002). A more recent example of that was the Kinetic North initiative ${ }^{17}$ to redevelop the NDSM wharf, located at the heart of Buiksloterham. Formerly home to one of the largest shipbuilding companies in the world, the site went through a series of dramatic transformations over the past thirty years. Following the rough times of NDSM's collapse in 1984, it took almost two decades for the abandoned shipyard to formally receive a new function and slowly become part of Amsterdam's new cultural scene. The founders of Kinetic North were a group of artists, skateboarders and sustainable entrepreneurs with tight connections to the squatting movement. Many of them have previously been evicted from other sites in Amsterdam and thus joined their efforts to turn the NDSM premises into a vibrant creative hub. As such, back in 2000, the founding group drafted a development plan ${ }^{18}$, which was soon to be endorsed by the municipality under the Breeding Places Amsterdam (BPA) programme (Pruijt, 2004:700). Since then, NDSM has grown into a cultural hotspot, which accommodates within the old industrial halls and upcycled shipping containers a thriving community of artists and entrepreneurs.

In 2012, NDSM's main hall dubbed the container city was also the launch pad for Metabolic, a cleantech start-up soon to become a key partner in the development of De Ceuvel19. During that same year, the on-land harbour concept won the idea competition organised by the municipality for a ten-year lease of De CeuvelVolharding's premises. Given the fairly short lease and contaminated soil, the design concept envisioned by Space \& Matter architecture studio revolved around notions of mobility and creative reuse. This included a flexible arrangement consisting of upcycled houseboats, rather than conventional buildings, and a phytoremediation project developed in collaboration with Delva Landscape Architects and the University of Ghent. Shortly after the plan was approved, the houseboats to be turned into ateliers and office spaces at De Ceuvel could already be spotted by the NDSM wharf, where they were partly retrofitted with the basic equipment required for their new function. At the end of October 2013, the houseboats were towed to De Ceuvel-Volharding site

\footnotetext{
${ }_{17}$ According to Eva de Klerk, founder of Kinetic North: http:/ /www.evadeklerk.com/ndsm-werf/

${ }^{18}$ Details of the plan can be found at: http://www.templace.com/tool-pool/onecfe2.html?tool_id=4073

${ }_{19}$ More details on Metabolic's connection to NDSM: http://www.evadeklerk.com/metabolic-2/
} 
and craned into their designated position on ground. Due to the financial constraints, as well as the site limitations, reusing the discarded houseboats proved a brilliant choice, which was to exceed even the most optimistic expectations on how the project will deliver on its promise. As one of the architects from Space \& Matter points out:

"I knew that houseboat owners are stuck with their old houseboats when they buy a new one and since there's no place to store them, many end up at junkyards. We came up with the idea to use them as basic structures to build upon and we could get them very cheaply (...) The winner of the competition got the right to use the heavily polluted 4,600-square-meter plot for a period of ten years. But we didn't receive any money and there were no buildings, so our plan had to be flexible, cheap, and off the grid as we didn't want to touch the soil." (Pop-Up City, 2015, emphasis added)

Right from an early stage, the on-land harbour set-up and the phytoremediation project - supposed to function also as a park for residents and visitors - received another valuable addition with the concourse of Metabolic. The cleantech firm became responsible during the implementation phase with designing an integrated technical and environmental system for energy, water and food production at De Ceuvel. As such, Metabolic's involvement has provided a new dimension and overarching vision to the initial plan. Labelled the Cleantech Playground, this merges both low-tech and high-tech solutions to turn De Ceuvel into a self-sufficient community and testbed for a small-scale circular economy. According to its founders:

"The Cleantech Playground is both a decentralized cleantech utility and a demonstration and testing site for new technologies that can transform how we produce and consume resources and public services in cities. Throughout the site, solar technologies will convert energy from the sun into heat and electricity. Green roofs and water collection systems are designed to collect, purify, and store rainwater for when it's needed. Sanitation systems will extract energy, nutrients and water from the waste produced for on-site food production. A network of sensors provide information on performance and user behaviour" 20 .

Merging the three interrelated areas of intervention stimulated a broadly inspiring narrative of creative reuse, experimentation and play. Hence, the various volunteering campaigns and events organised to promote the approach taken at De Ceuvel fostered a sense of community even before the founding partners could intervene in the area itself ${ }^{21}$. However, in spite of all attention and support the plan managed to secure on a rather short notice, its implementation moved forward in fits and starts. The financial and logistical constraints in particular, required inventive ways of bending the building

\footnotetext{
${ }^{20}$ Description previously provided on De Ceuvel's main webpage. Now available at: http:/ / thisbigcity.net/turning-houseboats-into-a-creative-eco-hub/

21 Aspect highlighted by most respondents from the community.
} 
codes and bypassing urban regulations, to allow for the use of experimental and often low-tech solutions.

Here, the very idea of De Ceuvel as playground is strongly suggestive of the shifting spatialities of experimentation and negotiation that enabled a sense of belonging and creative/collective action for community members. As one of the Metabolic founders contends in an interview for The New York Times, "[t]o follow through, you actually have to break a bunch of laws" (Schuetze, 2014). While the plan set-up as a regenerative urban oasis appealed to the municipality, coping with the many challenges related to licensing and approval, especially in the case of utility provision, proved a thorny task. In other words, the logic of flexible, cheap, and off the grid was not entirely consistent with the city regulatory frameworks. The need to identify alternatives was acknowledged by all sides, though it involved some intensive lobbying and a series of mutual concessions on the experimental boundaries that the community could afford $^{22}$. This required a fluid sociotechnical arrangement, thus able to seep through and even alter the overly dense meshwork of urban regulations. Pointing to this aspect, a community member explains that "what made it all possible is really the government, relaxing, letting go...and then you can see what these grassroots initiatives pretty much can do... it is not a top-down world anymore, it is a bottom-up world" (interview, emphasis added). In line with Bulkeley and Castán Broto (2013:373), "recognising experimentation as a site of politics" calls for a closer attention to "the emergence of new sites and intermediaries, which do not fit neatly established categories". De Ceuvel's broadly inspiring vision describes such a hybrid dynamics, through its catalytic effect on a whole range of future imaginaries and ad hoc coalitions to challenge established practices around sustainable, creative and inclusive citymaking. The plan's popularity, which featured also extensive media coverage over the past years ${ }^{23}$, was foremost driven by the capacity to morph these emergent imaginaries and transient actor constellations into a largely improvised collaborative arrangement. The latter, though its ethos of creative reuse, enabled a host of productive latencies to generate new material and affective dispositions that both subsume and exceed the residual materialities informing De Ceuvel's making.

\section{V.3.2 Warping materialities and affects}

As previously pointed out, creative reuse particularly concerns the residual surplus generated along dominant processes of accumulation and value production. In De

\footnotetext{
22 As pointed out by Eva Gladek, founder of Metabolic, the "site has no gas and no sewage connection; that itself is illegal". There were problems also with rainwater collection to be recycled into drinking water, which "was blocked by the city because that would have meant licensing the community as a drinking water provider — which was too complex and costly" (Schuetze, 2014)

${ }^{23}$ Resulting from the review of over sixty media articles
} 
Ceuvel's case, this was formative of an alternative value regime and related hybridisation processes that called into question notions of novelty or inventiveness. The creative assemblage of the on-land harbour, the phytoregenerative park and the cleantech playground is indicative of De Ceuvel's liminality, as well as of its potential to foster alternative conditions of possibility. As such, the creative reuse of industrial wasteland and all sorts of waste materials becomes a differential element, which transcends circumstances of disposal or dissolution in exploiting what Latham and McCormack (2004:719) call "the productive potentialities often hidden within the materialities of the urban". Animated by the circulation of heterogeneous ideas and materials, creative reuse interventions often entail unexpected functional couplings that reveal ingenious modalities to think beyond the taken-for-granted status of things. Take, for instance, the account provided by one of Café De Ceuvel's (figure 9) representatives:

"The whole café has been built by the architect completely upcycled. For example these poles are old bollards that were used in the water, in the harbour of Amsterdam, so they were there for eighty years in the water and now they are a construction. This, where we are right now, used to be a beach pavilion used for the emergency service in The Hague, and now we shipped it here and used it to build the café. But also the floor, for instance, is made of these old planks where bricks would be baked on...this is from an old gym, everything is upcycled or given a new goal' (interview, emphasis added).

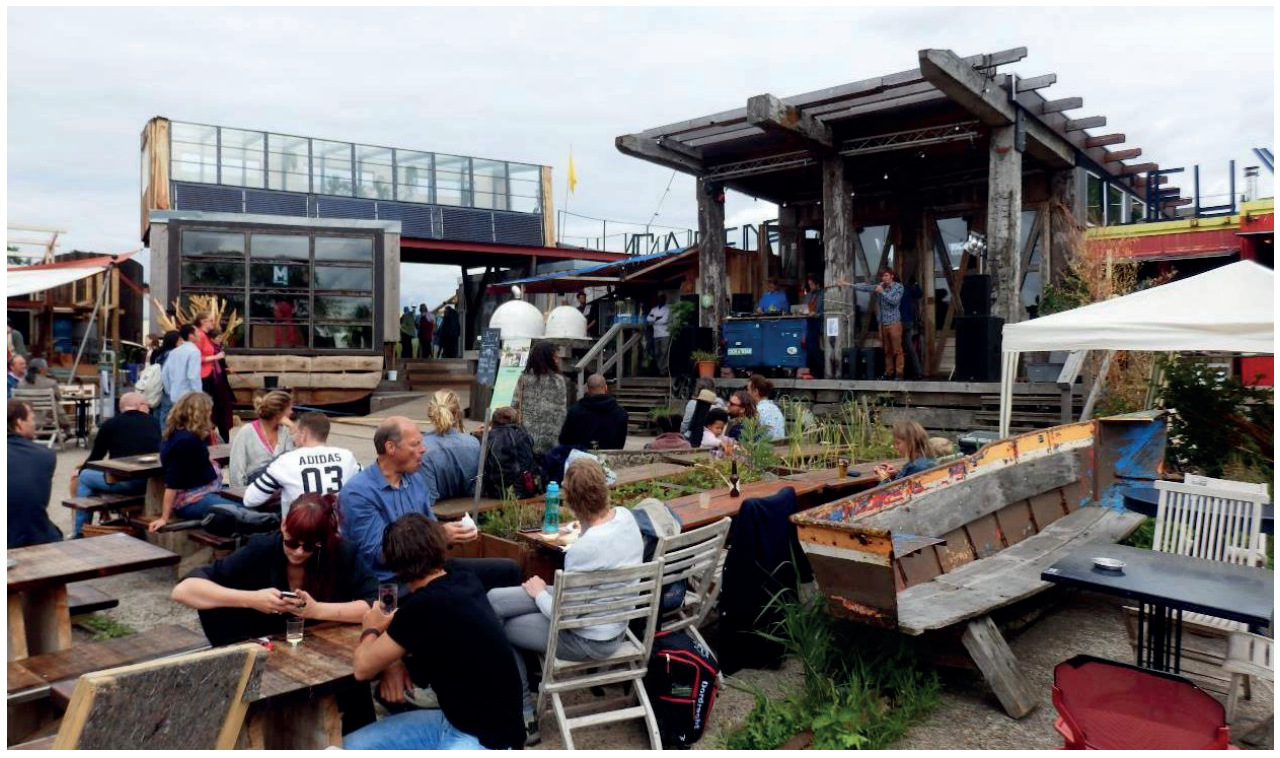

Figure 9. Cafe De Ceuvel (right) and Metabolic boat (left). Source: The authors

The on-site café and upcycled houseboats expose the disruptiveness of creative reuse as an alternative imaginary to creative destruction and its sway over processes of commodification. By warping a whole range of spatiotemporal conditions that allow 
for discarded things to make surprising returns, creative reuse interventions allude to non-linear hybridisation processes and modes of (re)ordering that put the squeeze on the functional status of things. As Moore (2012:792) convincingly suggests, "[w]aste can escape and exceed, not just our categories for it, but also the physical limits and boundaries imposed on it, and is given capacity to act on society in interesting and surprising ways". This is also the case with other emergent initiatives connected to the cleantech playground. With implementing an integrated system for food, water and energy production, the community seeks to close as many loops as possible in showcasing the viability of such a circular economy model. As emphasised by one of the respondents:

"We are really looking for those ingredients that are most innovative or most sustainable, for instance, we are using a lot of mushrooms which are from urban farming here in Amsterdam, and actually reusing waste streams from the city to grow within the city (...) We are now finding ways to work with local farmers to get their vegetables, and we have just finished the electric cargo bike to ship them. For the future we are looking into how to make it even more sustainable, so we are working on a biogas boat. This summer we will launch a crowd-funding campaign for the biogas boat so we can use our own organic waste, but also the organic waste from our local beer brewers, to digest these into gas that we can cook on. And we will do this on a boat actually, so we could also ship the boat during events to the city to showcase this technology. (...) We are also starting a garden on the island made of recycled plastic [figure 10], floating here, and we already had a volunteer day a few weeks ago to start planting seeds" (interview, emphasis added).

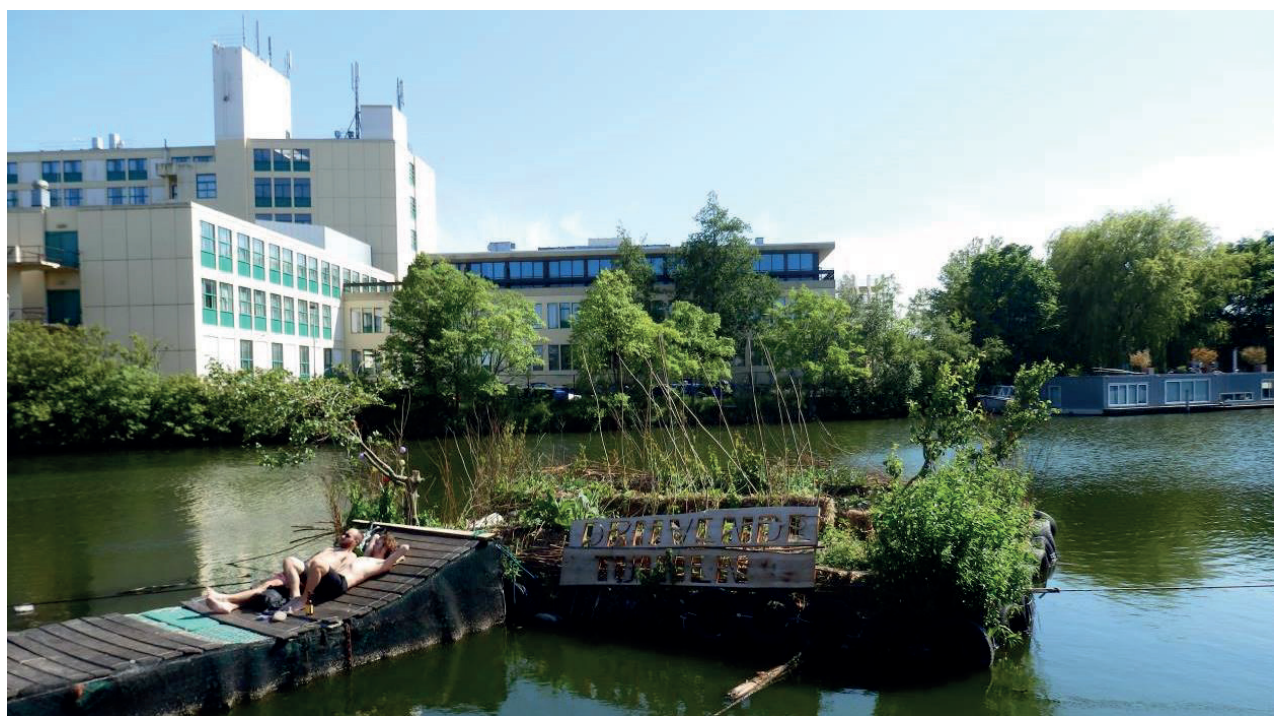

Figure 10. The floating garden at De Cenvel. Source: The authors 
Analogously, the houseboats recovery and the overhauling of their materiality flag the alternative conditions of experimentation and attunement occasioned by the flexible, cheap, and off the grid rationale. Through a mixture of both rudimentary and advanced technologies coupled to their new functions, the houseboats metamorphosis resembles a tinkering processes, (still) open to all sorts of tweaks and additions that would make them even more sustainable. Notably, the ways they were transformed mirrors in an intriguing manner the very sociomaterial dynamics, which inspired the development of a sense of community at De Ceuvel. During the preliminary phase, when the houseboats were retrofitted with the basic equipment and moved on-site, prospective tenants were invited to write a motivation letter on how they envision their role within the community, as well as how they would customise their new workplaces. The initial selection procedure was organised via Marktplaats, one of the most popular auction websites in the Netherlands. As indicated by most people with an office at De Ceuvel, the initiative was well received, as they had the freedom to improvise in customising the houseboats to their own needs, while coupling also their personal projects to the broader community agenda. Referring to the moment when the houseboats revamping was nearly completed, one of the residents cheerfully recalls that "somewhere in April, there was this point, when they hung the rope swing in the tree and then I had the feeling that it was enough for people to finish this...for me the rope swing was a symbol, we do not only have to work, there is also time to play..." (interview, figure 11).

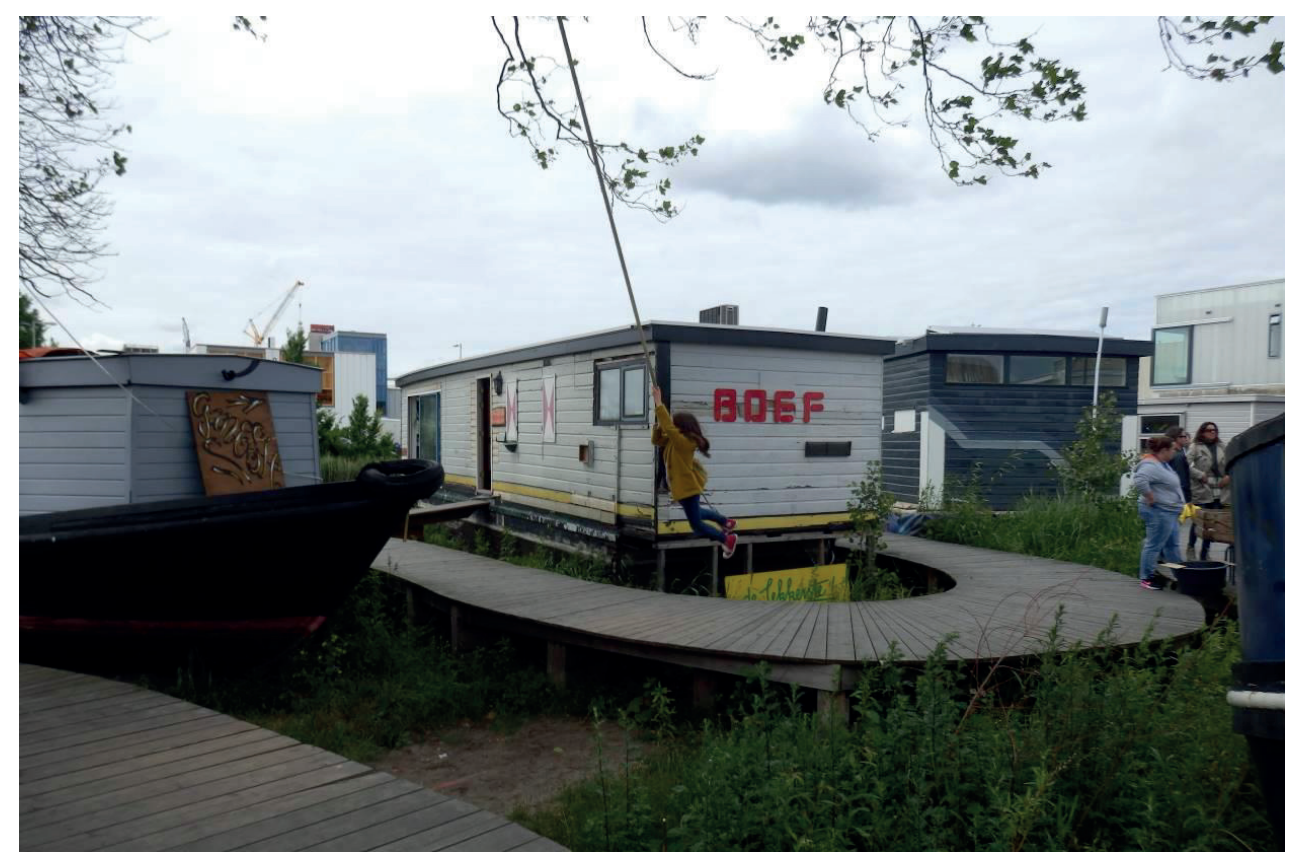

Figure 11. The rope swing. Source: The authors 
Redolent with De Ceuvel's liminality, enrolment through play emerges as an important dimension of creative reuse, especially in maintaining its particular 'affective atmosphere' (Anderson, 2009). The latter provides the circumstances to rehearse alternative modes of assembling the urban and, concurrently, to enable further processes of creative production (Rantisi and Leslie, 2010). In tune with the previously evoked fluid/ navigational metaphor, these transient enrolments through play generate interstitial spaces of loose connections, at once familiar and strange. Distinguishing as the norm rather than the exception, enrolment alludes here to the multiple translation processes that are the ebb and flow of the chase to recalibrate this between-ness "as active points of reference, connection and anchorage" (Simone, 2013:243, emphasis added). For instance, when asked to account on De Ceuvel's popularity, a community member explains that:

"There is a project very much alike at NDSM, where they transformed a big industrial hall for artists to establish their own studios and that you could basically do in all industrial halls around the world. But the houseboats are something typical for Amsterdam that you would not do in most of the other cities in the Netherlands, definitely not in Paris. The industrial halls could serve as an example on how to organise a project like this. It paves the way for this, on how you can build a sort of community or broedplaats [breeding place]. Whereas here, it goes well beyond creating workspaces for creative people. The ambition is way bigger, and the way it is shaped is also way more surreal" (interview).

On a similar note, another respondent emphasises the inspiring character of De Ceuvel by pointing to its equally unconventional and familiar atmosphere:

"I mean the idea is totally genius, to put the houseboats on toxic earth, with plants that will clean it up. And it fits the historic context of Amsterdam because we have boats, navigation...Not only the concept, it is altogether. When you walk in the area it looks fantastic, it immediately inspires people because it is so unconventional. But it is not far away from our unconscious imagery, it is not out of place you know...The old boats that are lying here fit the image of Amsterdam" (interview).

Identified with Amsterdam's legacy of living by the water, the creative reuse of the discarded houseboats and of the derelict shipyard stands out as an expression of the potent afterlives of things and affects (Ash, 2014:7; Thrift, 2008:9). Concurring to this line of reasoning, Hill (2015:413) contends that the "always already affective nature of matter and the material opens up opportunities to think through the role of past in the present from an entirely novel perspective, to embrace the immaterial as part of the material, and to reanimate the past". Accordingly, De Ceuvel harbours a fluid sociotechnical arrangement, where improvisation and play unveil new conditions of possibility to warp the temporality and functional status of things. Through often unexpected synergies and functional couplings, the on-land harbour/playground 
pitches under the topological inflections that allow all sorts of seemingly residual materialities and affects to make surprising returns and, as such, to unlock alternative future trajectories.

\section{V.4. Topologies of creative reuse: transient fixed points and shifting spatialities}

To follow on the points raised so far, we dwell here on what a topological reading of creative reuse, informed also by De Ceuvel's case, might add to ongoing conceptualisations of urban materialities and city-making processes. As emphasised throughout the article, creative reuse pertains to those action repertoires inspired by the residual surplus generated along dominant processes of accumulation and value production. While animated by a logic of improvisation that is politically Other to the realities of creative destruction, creative reuse also exposes its somewhat paradoxical symbiosis with the former. However, it does this by constantly dogging the linear entrapments of historical and functional redundancy to expose the problematic and, most often, productive latencies scored across established norms and practices. This kind of dynamics, which relies on all sorts of tinkering processes to stimulate alternative imaginaries, turns De Ceuvel into a place "of inspiration incorporating many possible worlds" (Thrift, 2006:291). Along the aspects signalled in the empirical section, the on-land harbour and cleantech playground are subject to shifting instances of mobility and stillness (Cresswell, 2011), contingent on the intensity and rhythmic qualities of their various enactments. Somewhere else, in the attempt to capture the interplay of contingent imaginaries and how they warp various conditions of encounter, this dynamics was envisioned as resting upon "topologies of fixed points and shifting spatialities" (Barba Lata and Minca, 2016). Consequently, there is the question of what would fixed points have to do with the otherwise processual reading offered thus far?

Rather than alluding to the idea of fixity or permanence, topological fixed points pertain here to the dislocation of organising centres as informed by the proliferation of contingent imaginaries. In other words, as Massey (1995:184-185) suggests "the past of a place is as open to a multiplicity of readings as is the present". This idea resonates, among others, with Benjamin's (2002:883) treatment of the "Copernican revolution in historical perception", or with Tarde's (1903:207, emphasis added) perspective on "progress from within to without". In tune with the issue raised earlier on the linear entrapments of historical and functional redundancy, creative reuse interventions transcend circumstances of disposal or dissolution in giving a spin to all kinds of material and affective dispositions. As it results from the empirical material, the latter entertain the emergence of alternative imaginaries, as with the houseboats customisation, the shifting subjectivities of De Ceuvel's residents in connecting their 
personal projects to the broader community agenda, or the constant tweaking of sustainable technologies implemented on-site. These multiple and contingent imaginaries are strongly suggestive of an anticipatory politics, one in which they subscribe to an ultimate goal roughly defined and constantly reworked in the chase for a better-yet-to-come. A topological reading would thus prove lucrative in unveiling the multiple inflections that such imaginaries afford in interfering with each other to enable the emergence of alternative value regimes and urban materialities.

To clarify the reference to topologies of fixed points and shifting spatialities further, topological fixed points could be understood as snapshots of the apparently stable centres of funnelling flowing surfaces. They only make sense in relation to the imaginaries that (re)animate particular material and affective dispositions, or in other words, to the shifting instances of core and periphery, inclusion and exclusion, mobility and stillness they bring into existence. In synergetics topology, fixed points are a common feature of any "fluidly bestirred system" for which they act as "constants of topological inventorying" (Fuller and Applewhite, 1979:371). Concerning the matter of alternatives, the plurality of co-emergent imaginaries is what interests us the most. Articulated by a logic of aspiration and differentiation, these imaginaries are subject to rhythmic qualities which seem to escape the sole grip of spatial metaphors. Hence, the syntagm topologies of fixed points and shifting spatialities (always in plural) is a direct reference to the varying dynamics of temporal and functional contingencies that keep redefining narratives of belonging and repertoires of creative action. In line with Crang and Travlou's (2001:175) topological interrogation of memory, "[p]laces of memory stand inserted simultaneously in a past order and the present", and as such "they offer cracks in the surface of the present where time [and things] can be otherwise". Moreover, we would then argue, the simultaneity of multiple attempts to recalibrate present conditions of assembling the city, is as much a function of past as is of future imaginaries. And this is the stuff from which alternative city-making trajectories are extruded in reanimating the sediments of seemingly established orders.

\section{V.5. Conclusions}

In this article, we have offered a conceptual and empirical interrogation of creative reuse, as an alternative imaginary informing innovative responses that are politically Other to, yet inseparable from, the realities of creative destruction. The argument was threefold. First, we have shown how creative reuse distinguishes though its ethos of improvisation, specifically concerned with the residual surplus that results along dominant processes of accumulation and value production. Second, by dwelling on De Ceuvel's case, we have explored multiple instances of creative reuse. These suggest that by challenging circumstances of disposal or dissolution, creative reuse interventions provide 
inventive ways to expose and alter the productive latencies of dominant sociotechnical arrangements. As such, things that were otherwise redundant/disposed/forgotten can stimulate new material and affective dispositions that call into question established norms and practices. Third, in attaining to the non-linear dynamics of creative reuse interventions, we have described these as relying on topologies of fixed points and shifting spatialities. The latter represent a salient vehicle to navigate the simultaneous interplay of contingent urban imaginaries, also in support of the argument that the emergence of alternatives is redolent with both the enactment of past conditions and of related future imaginaries. 



\section{VI.The Magic Mirror II: Floating churches, stranded houseboats and other surprising (re)turns*}

"Nothing ever begins. There is no first moment; no single word or place from which this or any other story springs. The threads can always be traced back to some earlier tale, and to the tales that preceded that...Nothing is fixed. In and out the shuttle goes, fact and fiction, mind and matter, woven into patterns that may have only this in common: that hidden amongst them is a filigree which will with time become a world'. (Barker, cited in Smith, 2003:561)

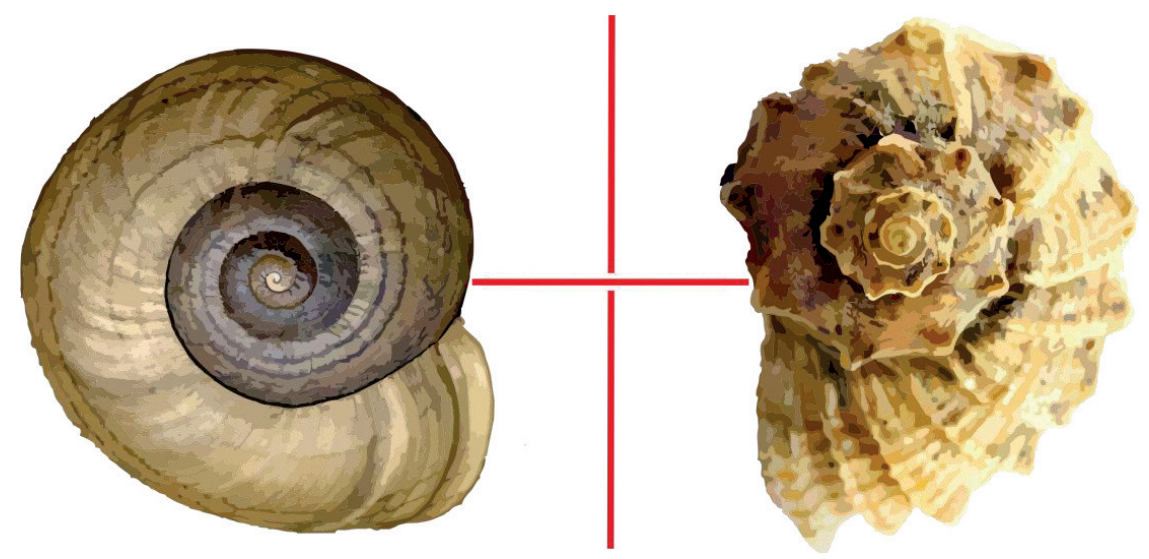

*This chapter includes excerpts from the review published as:

Barba Lata I (2014) Review - Rob Shields' Spatial Questions: Cultural Topologies and Social Spatialisations. Society \& Space open site: https://societyandspace.com/2014/10/02/shields 


\section{The Magic Mirror II: Floating churches, stranded houseboats and other surprising (re)turns}

At the outset of this conceptual and empirical exploration I evoked Escher's Magic Mirror as an inspiring companion to account on the displacements that veer between two innovation modalities: creative destruction and creative reuse. This has involved a moreor-less experimental interrogation of the in-betweenness of what appears to be another inescapable binomial redolent with the legacy of Western thought. In tune with the arguments presented in The surface and the abyss, I subscribe to Châtelet's (2000) and Deleuze's (1994) treatment of the problematic as an expression of multiplicities and a fertile source for usurping axiomatic distinctions from within, including the ones that animate the current enquiry. First of all, these concern the threefold split drawn in the introduction chapter between the meanings, workings and expectations related to various innovation imaginaries. Along this line of reasoning, the relation between innovation and imitation was another key distinction discussed at length in The Magic Mirror I. Connecting the former and the latter, the question of retroactive inspection - signalled by both Schumpeter and Girard as a lucrative strategy to tackle the impact of innovation processes at a given time (see chapter II) - provides the nub of the aspects addressed further in this section. These apply to the emergence of alternatives or, in other words, to the multiple conditions of possibility of linking 'past' conditions to 'future' imaginaries. To place these issues within the broader ambit of the dissertation, I now turn to an overview of the mirroring instances presented thus far, also meant to ensure the transition to the conclusions chapter. There are three interrelated points. They cover the alternative imaginaries, the topological treatment and the observation repertoires mobilised to investigate the two innovation modalities designated above.

\section{VI.1. Alternative imaginaries of creative reuse}

The first point touches upon the alternative imaginaries enabled by creative reuse and their sway over innovative arrangements that often override a broad range of normative assumptions. Hence, the cases of The floating churches of Volgograd and $A$ barbour on land unveil multiple displacements scored across formal and informal practices, and related instances of core and periphery, inclusion and exclusion or mobility and stillness. As shown in chapter IV, through the creative reuse of discarded ships, forms of religious ritual and the oblast riverscapes, the floating churches of Volgograd eroded previously held distinctions between the secular and religious, 
mobility and fixity, absence and presence, in patching up a constellation of sites completely stripped of their religious infrastructure. In a similar manner, the case of De Ceuvel in Amsterdam evidenced how the creative appropriation of wasteland and abandoned houseboats, rudimentary and advanced technologies, became the drive for makeshift sociotechnical arrangements that challenged established practices around sustainable and inclusive city-making. Informed by processes of accumulation that exploit the residual substrate of things and affects, these interventions move beyond the sole exposure of Othering instances. They reveal inventive ways of reaching the critical mass that would trigger the emergence of alternative innovations. In spite of their contextual specificity, such alternatives generically owe their provenance to synergies performed across heterogeneous domains, often leading to peculiar hybrid formations. The latter, allude to the dislocation of organising centres through their material and affective dispositions, which habitually rely on the productive latencies that result along dominant processes of accumulation and value production.

Merging the main lines of argument from chapters III, IV and V, the title of this chapter already provides an indication of how thinking through the magic mirror motif offers a clearer perspective on the workings of creative reuse. From the genealogical interrogation of topology to the unconventional interventions discussed in the empirical sections, creative reuse emerges as the vehicle of surprising returns. As previously suggested, these enable a more generous reading that transcends the immediate affordances of mere repetition or circumstances of disposal - one that pivots on the key role of variation through mimesis or the potent afterlives of things and affects in animating alternative forms of innovation. Here reference to alternatives should be understood both in relation to the dominant narrative of creative destruction, as well as to how various imaginaries - whether digested as secular, religious or otherwise become entangled and mirror each other in intriguing ways. Consequently, even when proceeding from the fairly basic distinction that things envisioned as fixed end up afloat and travelling around, as much as things expected to float and travel around become stranded, the idea of surprising returns opens a broad spectrum of meanings and potentialities. And as such, the resulting instances expose realities that are much more turbulent than commonly believed.

Furthermore, these seemingly odd occurrences are subject to proximities and synergic effects that defy linear interpretations of materiality or temporality. As emphasised in the empirical chapters, creative reuse interventions move beyond the linear entrapments of historical or functional redundancy in exposing the productive latencies scored across established norms and practices. The resulting spatial and temporal proximities come close to what Michel Serres describes as a dynamics of percolation, one that eludes linearity by "conveying wormholes of sheer acceleration, bottlenecks of stoppage or equilibrium, zones of stationary values" (Serres and Adkins, 2012:377). Dissolved 
through these non-linear progressions is an entire fascia of taken-for-granted assumptions, which morph into alternative narratives of belonging and creative action. Along this line of reasoning, Serres (2008:81) contends that "[e]verything meets in contingency, as if everything had a skin". Dwelling on the notion of mixture as an expression of confluence, unfurling and bybridity, he suggests further that:

\footnotetext{
"Contingency is the tangency of two or several varieties and reveals their proximity to each other. Water and air border on a thick or thin layer of evaporation, air and water touch in a bed of mist. Earth and water espouse each other in clay and mud, are joined in a bed of silt. The cold front and the hot front slide over each other on a mattress of turbulence. We live on slow, inexorable moving footpaths, thousands of meters beneath our feet." (Serres, 2008:81)
}

Serres' elemental philosophy saliently captures the interplay of contingent imaginaries and the multiple inflections they afford in interfering with each other, as evoked in $A$ harbour on land. Aligned to the focus on amphibious practices, the instances of creative reuse explored so far could be envisioned as belonging to a veritable amphibious realm of 'clay and mud'. Accordingly, notions of value shift across resulting interfaces, where creative reuse operates both as source and extension of the much narrower axiomatic of creative destruction. Envisioned in this manner, one would expect to find on one side of the magic mirror the more immediate cyclical progressions of creative destruction and, on the other, the realm of mixtures, 'mothballing' (Lorimer, 2007) and 'wormholes' (Sheppard, 2002), set in motion by the percolating dynamics of creative reuse. Nonetheless, while maintaining the distinction between these mirroring realities might prove useful to pin down an otherwise intricate argument, the liminal spaces where creative destruction and creative reuse imaginaries collide and mix arguably hold the key to the genesis of most innovations. As in Lewis Carroll's (1920) Alice's Adventures in Wonderland, the rabbit hole emerges as a space of translation, which connects realms at once familiar and strange. These liminal spaces where worlds flow into each other become the magic mirrors that reveal the umbilical links between absence and presence, where "absence can instead be conceived of as a presence, with a different politics" (Steinberg and Peters, 2015:260, emphasis in original).

The magic mirror interface acts at this juncture as the fief of metaphor and metamorphosis, funnelling crossovers between contingent innovation imaginaries and their related functional domains. Both case studies from chapters IV and V expose a panoply of such crossovers that often generate new conditions of possibility for creative action. These transient couplings bear the mark of creative reuse, through emergent functions, which somewhat paradoxically are redolent with, yet persistently dog the realities of creative destruction. Understood in this manner, creative reuse moves beyond the overriding logic of creative destruction, scoring as its inescapable complement that always ushers the making and unmaking of things. Inspired by what was labelled in 
chapter $\mathrm{V}$ as a logic of aspiration and differentiation, creative reuse imaginaries are subject to rhythmic qualities which seem to escape the sole grip of spatial metaphors. Consequently, these imaginaries coil both the making and unmaking of things through what seems to be a vortex effect (figure 12) that adds more depth to a surface reading of innovation cycles. And this brings me to the second point, which concerns the topological interrogation of the interstitial synergies evoked above.

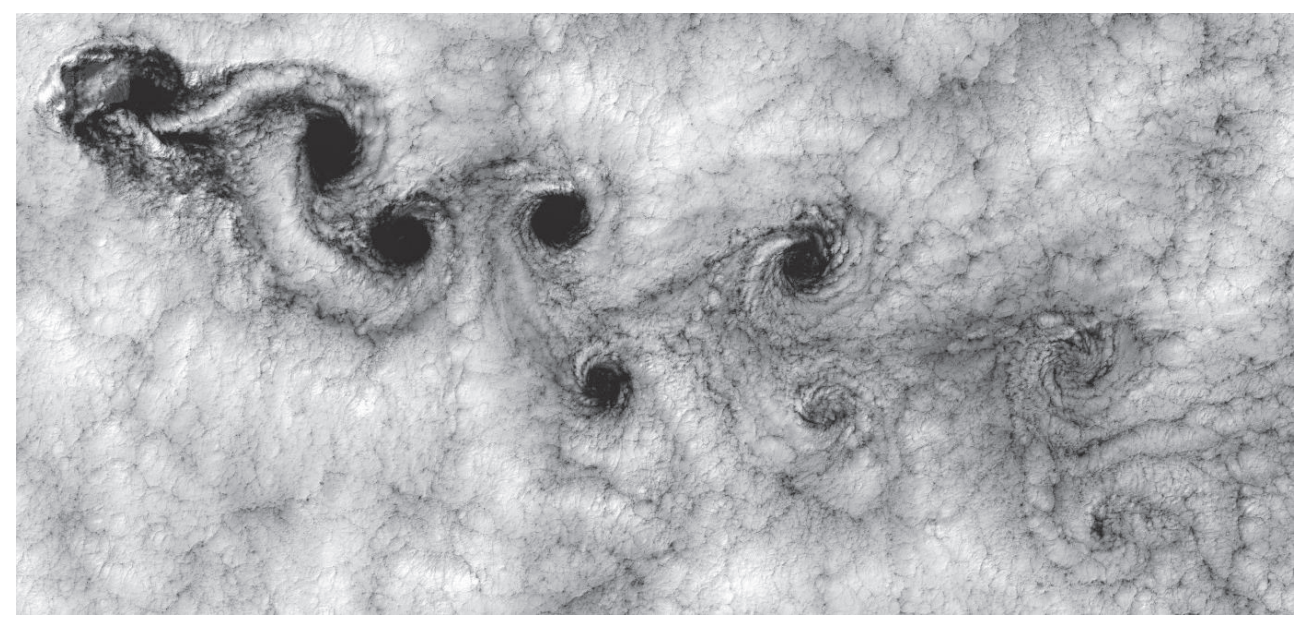

Figure 12. Fluid dynamics: string of vortices caused by wind flowing around the Juan Fernández. Islands. Source: Bob Cahalan, NASA GSFC, public domain image

\section{VI.2. Topologies of fixed points and shifting spatialities}

Throughout the previous chapters, I pleaded on several occasions for a processual reading of creative reuse, inspired by topologies of fixed points and shifting spatialities. These have been used particularly to account on the recursive functions that foster the emergence of alternative innovations. To revisit Tarde's (1903:207) perspective on "progress from within to without", presented in Magic Mirror I, recursive functions are suggestive here of the underrated role of variation through mimesis. These are the evolutionary pathways that mimic the ideas and ends of particular innovations, leading in turn to the proliferation of alternative expressions and means. Once these forking pathways enter more stable trajectories defined by some specific principle of continuity, they generate new spatiotemporal conditions of encounter, as with the operative areas of Volgograd's floating churches or the on-land harbour in Amsterdam Noord. In view of that, recourse to topological thinking within this research was primarily driven at unpacking the interplay between functions and the spaces they articulate. Their analysis has drawn on notions of temporality and warping, to expose the attunement of functional domains of varying dynamics. 
Along this line of reasoning, chapters IV and V highlight the counterintuitive logic of topological fixed points, in acting as transient organising centres that mimic the progressions of vortices and eddies. As Fuller and Applewhite (1979:371) tell us, in synergetics topology any "fluidly bestirred system has two opposed polar points that do not move" and that "become constants of topological inventorying". Hence, topological fixed points could be understood as snapshots of the apparently stable centres of funnelling flowing surfaces. In other words, they appear as fixed, yet they are always on the move, always evolving and interfering with other 'flowing surfaces', as illustrated by the string of vortices in figure 12. Conversely, they only make sense in relation to the imaginaries they give a spin, or otherwise, to the shifting instances of core and periphery, mobility and stillness, they bring into existence. On a similar note, Châtelet (2000:166, emphasis in original) dwells on the axis-loop system in electromagnetism, to point out that lines of force as "neither real nor artificial" irrigate space allowing "virtuality to reclaim its rights". This is perhaps where the magic mirroras expression of the transient liminal spaces of collisions and mixtures - proves most resourceful in unveiling how different innovation imaginaries and their operative domains interfere with each other. When considered through its reflective (surface) and permeable (depth) properties, the magic mirror becomes a dynamic operator that mediates the swirl of seemingly incompatible processes of varying magnitudes, rhythms and momenta. Its logic of liminality and metamorphosis pivots on topological qualities that allow emergent interstitial spaces to contract and expand under external forces.

Drawing on Clerk Maxwell's thought experiments, which led to the Classical Theory of Electromagnetic Radiation, Châtelet evokes the idle wheel as device that reduces frictions by inducing a translation effect between opposed revolutions. It thus operates as "a technology for setting up similarity" that "is essential to concentrate all attention on the articulation and on its capacity to find two motions in one mobile point, just as the metaphor captures two ideas in one without ever mixing them up" (Châtelet, 2000:180-181). Analogous to the idle wheel dynamics, the interstitial spaces resulting in-between dissonant functional domains act like catalysts mediating the centripetal and centrifugal forces, identified by Thom (1989:254; see also, Thompson, 1945) as a fundamental condition in the making of all things. Thus, beyond attending to the fluid dynamics of creative reuse, the syntagm topologies of fixed points and shifting spatialities (always in plural) strikes the nub of another related matter - the co-emergence of innovation imaginaries with their environments and the ways they interfere with other contingent imaginaries.

As soon as creative reuse and creative destruction imaginaries are acknowledged as closely interlaced, innovation processes could be generically accounted for in terms of developments that subscribe to an ultimate goal roughly defined and constantly reworked in the chase 
for a better-yet-to-come. This is the stuff from which alternative future trajectories are extruded in reanimating the sediments of what appear to be irreconcilable orders. When wrecked landing ships turn into travelling churches and stranded houseboats become hype offices, mirror matter takes its toll by unveiling constellations of silent Others that most often make surprising (re)turns. Subsequently, once stabilised, these constellations flag the topological fixed points of emergent functional domains that funnel a whole range of latent potentialities into innovative arrangements. In line with Daniel Miller's (2011:23) comment on the taken-for-granted status of things, "it is only when the juxtaposition or material is distinctly odd that we are shocked into an awareness of the underlying technology". As with the idle wheel translation effect, the liminality of the magic mirror as mobile interface between grounded and more fluid readings of innovation leads to the third point on observation repertoires, of what one observes and how one observes topologically.

\section{VI.3. Observation repertoires}

In light of the methodological considerations from the introduction chapter, the discussion of observation repertoires completes the loop between the two Magic Mirror sections. Here the main concern is with the dynamics of observation in relation to the dynamics of innovations, and phenomena more broadly. Since modalities of enquiry always articulate a particular cut, "we might strategically choose who or what to hold to account as an ethical or political stance" (Anderson et al. 2012:186). Among the range of strategic options highlighted in the introductive notes on methodology, I pointed to diagrammatic reasoning as a resourceful modality to unveil alternative instances concerning "unformed traits capable of combining with one another"(Deleuze and Guattari, 1987:145). This has resulted into the overarching concern with topological grammars in prioritising the role of relations and synergies that enable innovative arrangements. According to Brian Rotman (2012:256), once diagrams are understood in terms of their performative dimension, i.e. as "embodied acts that bridge the gulf between thought and the sign", the focus shifts toward their topological properties to account on how materialities become knotted in different configurations (see also, Netz, 2010). The treatment of fixed points and shifting spatiatlities operates in a similar manner connecting observation repertoires, which contract and expand between grand innovation narratives and empirical accounts of micro-societal shifts.

In Spatial Questions, Rob Shields (2013:156) contends that "[t]opological approaches can take apart the static poles of ontology". And it is through the painstaking process of carving out these genealogies of meaning and their problematic dimensions that topology can undermine the taken for granted categories used in attaining to 
spatiotemporal and material conditions. As in Raphael's fresco The School of Athens (figure 13), this would allow distant figures to enter into dialogue even for an instant, with the Pythagoreans and the Peripatetic School to sit next to the astronomers of the Islamic Golden Age and Copernicus (Shields, 2013:50). Or for Euclid, Apollonius and Archimedes' diagrammatic reasoning to permeate the pioneering works of Hilbert, Klein and Poincaré in the framework of modern mathematics (Netz, 2003). Approached in this fashion, empirical explorations can move too beyond a solely chronological appropriation of historical conditions to redefine notions of novelty or innovation. As shown through the interrogation of the floating churches and the on-land harbour, tapping into the potent afterlives of things and affects proves a lucrative strategy to unpack the productive latencies that often animate creative action within and between various practices.

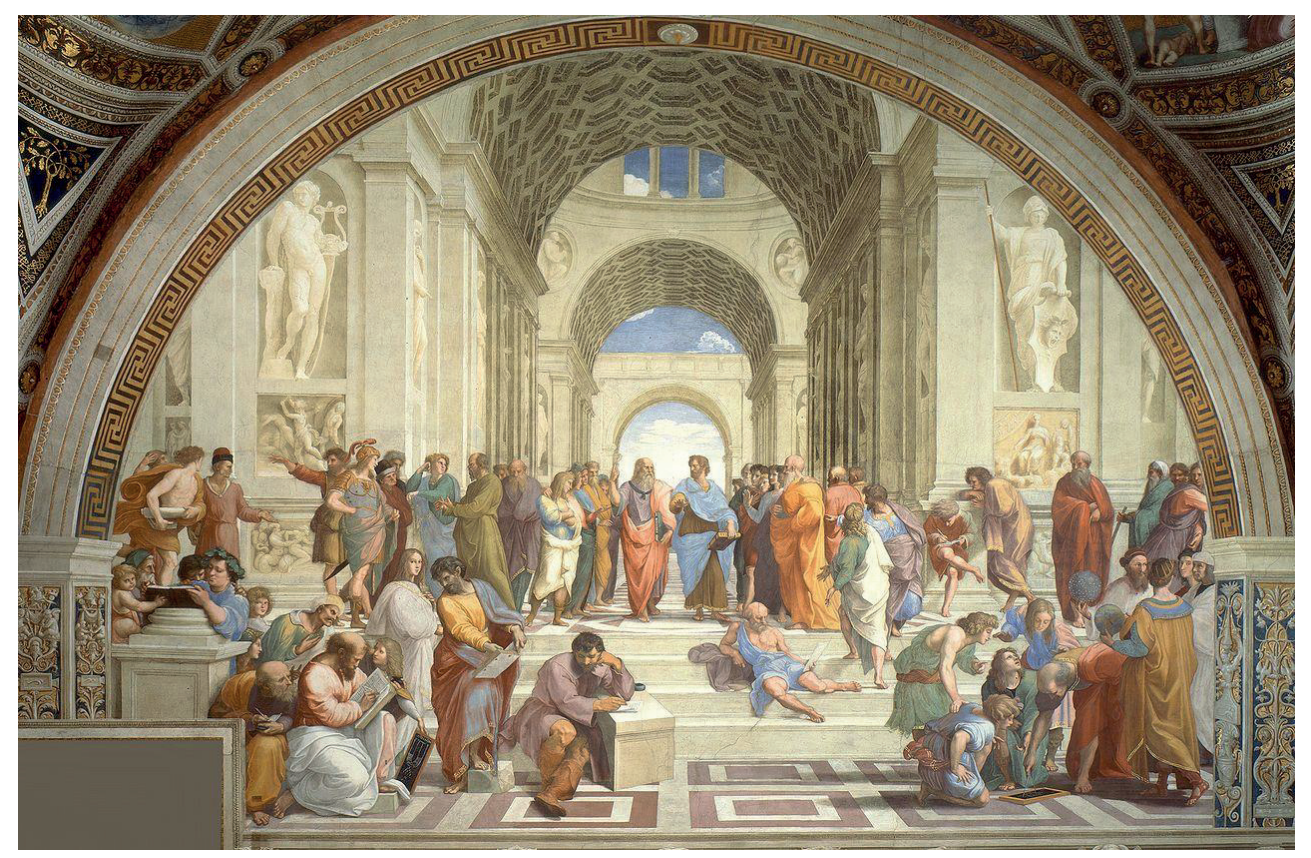

Figure 13 - Raphael's 'School of Athens' (1505). Source: Wikimedia Commons, public domain image

Shifting from grand narratives to alternative genealogies and empirical accounts, methodologies and related observation repertoires need to lend themselves to a dynamics that transcends the metrical logics of short paths and minimum resistance. Once symmetry is acknowledged as "a moving target" (Law, 2004:173), both in the unfolding of phenomena and modes of observing, translation processes can be performed more effectively. Michel Serres (2008:143) offers the maze motif, as an alternative to the metric discourse on method, in trying to find "the best way of creating most feedback loops possible on an unstructured and short itinerary". 
Accordingly, topological approaches articulate meandering paths which connect the constellations revealed through multiple modes of observing. This relates for instance to the conceptual exploration presented in chapter III or the hybrid sociotechnical arrangements discussed in chapters IV and V. They provide signals for the emergence of functional domains that draw seemingly established orders into amphibious realms of shifting value regimes and of fusion. Thus topological fixed points prove perhaps the most resourceful here in accounting on the interplay between modes of observing and the "schemas of operations and of technical manipulations" defining any practice (de Certeau, 1984:43). In other words, they provide the means to link the observations informed by various political dispositions to lines of action. And this leads me to a final remark on the question of surprising returns.

In Magic Mirror I, I referred to one of the main conundrums in studying innovation processes: how to tackle an otherwise future-oriented activity by overcoming its present axiomatic? Now, to attempt a partial answer to this question: the residual substrate resulting along the above lines of action enables a matrix of potentialities that is not entirely self-evident, yet packs the latent constituents of future innovations. Put differently, the residual belongs to the domain of the problematic, of things and affects fading into penumbrae and shadows. They might be forgotten or dismissed as redundant to emergent imaginaries, however they hold the seed of what was not-yetpossible to achieve or what was envisioned at some point as a better-yet-to-come. Therein lie the fixed points which will spark off actualisations that prove at once familiar and strange. Dwelling on the mirrored sculptures of Anish Kapoor, Marcus du Sautoy suggests that in spite of "the virtual world that these mirrors create, they are in fact a better reflection of the reality of the space we inhabit" (cited in Jones, 2014:2590). Among the truly outstanding innovations that the future holds, we will surely witness quite a few surprising returns, far more spectacular than the ones unveiled through the Magic Mirror companion of this research journey. For a sneak preview of that, we could start by paying more attention to the silent and, on occasion, peculiar Others... 



\section{Conclusions}

Accessories of emotion and purpose indeed accompany knowledge production, thus, as scholars, we should never cease reflecting on how past(s) and future(s) are continuously remade in our becoming... (A sequel to Whitehead's argument quoted at the outset of chapter I)

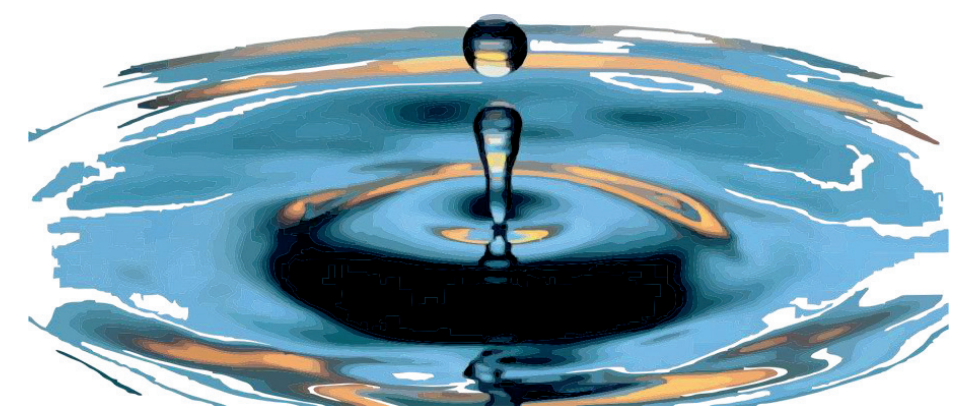




\section{Conclusions}

Having reached this point, which I would qualify as a pit stop instead of an end, it is time to sum up and pin down the key findings of this conceptual and empirical exploration. In retrospect, and to follow on the discussion from The Magic Mirror II, perhaps the best way to describe this dissertation would be as a journey of metaphor and metamorphosis. There are two main reasons for that. The first and most obvious, intimates the experimental and, on occasion, speculative modalities chosen to examine innovation. The second, pertains to the shifting roles which allowed me to perform this research across diverse filiations of Western thought, grand innovation narratives and ethnographic accounts of micro-societal shifts. Beyond trying to get across what might appear to some as a solipsistic report on how innovation works, this exploration certainly remains a political exercise. As such, besides the main research questions, there is still another important one pending. The latter concerns what it is that leads, or should lead, the way in such an interrogation? While at the outset of this journey I would have been more at ease with pointing either to empirical data or a theoreticallyinformed approach, admittedly, at this stage, a clear-cut answer would not feel that comfortable anymore. This conundrum has also dogged the fairly late definition of innovation processes provided in The Magic Mirror II, as developments that subscribe to an ultimate goal roughly defined and constantly reworked in the chase for a better-yet-to-come.

Formulated in the above manner, the reading offered to innovation processes appears to substantiate an endless exercise of anticipation. And indeed, this is partly the case. Once creative destruction and creative reuse imaginaries are acknowledged as intrinsically woven, it makes little sense to evoke beginnings and ends in normative terms. Accordingly, the definition encapsulates the plea to seek beyond the linear entrapments of historical and functional redundancy in exposing the problematic and, most often, productive latencies scored across established norms and practices. In other words, to pay closer attention to what others have eloquently coined, the potent afterlives of things and affects (Ash, 2014; Thrift, 2008). Rather than romanticizing creative reuse interventions, this is an attempt to grant them recognition as the inescapable complement to dominant processes of accumulation and value production. As pointed out in the introduction chapter, inventiveness - and the multiple inflections it affords through innovation processes - represents an essential feature of any practice. Consequently, the material and affective dispositions cultivated through the emergence of alternatives, within and between various practices, signal the dislocation work occasioned by processes of variation through mimesis. In line with the discussion from $A$ barbour on land, these emergent imaginaries rely on a logic of 
aspiration and differentiation, which allows them to interfere with, and shape each other or even morph into new narratives of belonging and creative action.

\section{VII.1. Navigating realms of loose connections}

In the introduction, I suggested that while innovation processes reflect the ebb and flow of any practice, established grand narratives fall short in recognizing the multiple inflections those innovations actually afford. Hence, the investigation took as a reference point the broad notion of alternatives, as instances that reveal other conditions of possibility and, in doing so, that challenge normative assumptions about novelty, value creation or materiality. This choice was informed by two main lines of reasoning. The first, drawing on the fact that alternatives are often to be recovered from the very dynamics of mainstream innovations, branching out beyond their original purpose, which flags the underrated roles of imitation and variation through mimesis. And the second, resting upon the basic assumption that both mainstream and alternative innovations are subject to varying dynamics and rhythmic qualities, more complex than commonly asserted through linear or yclical interpretations. Merging the two lines of reasoning, led me to describe this research as belonging to an amphibious domain of enquiry, suggestive of the experimental approach taken in connecting canonical interpretations to more fluid/unconventional readings of innovation. The amphibious conceptual imaginary dictated also the thematic repertoire and ambit of the case studies selection. This has resulted in the empirical focus on amphibious practices, which could be broadly understood as the referents of material and affective dispositions, as well as of narratives of belonging scored across land-water interfaces. Thus, the first research question formulated at the outset was: in what ways do different amphibious practices acknowledge the spatiotemporal and material conditions of innovation?

To use their obvious elemental liminality as point of departure, amphibious practices emerge as an expression of labile environments, where sociotechnical arrangements need to be easily alterable under frequently changing conditions. In terms of the meanings attached to innovation processes, both case studies reveal particular material and affective dispositions toward mobile technologies of dwelling. The latter are immediately apparent through the imaginative deployment of ships, houseboats and other floating stuff, in challenging more conventional ways of inhabiting land-water interfaces. On a more subtle level, it is interesting to notice how the liminal conditions of these practices facilitate the swift appropriation of all sorts of action repertoires that were not previously part of their "schemas of operations and of technical manipulations" (de Certeau, 1984:43). In turn, this augments their operative span across emergent domains of loose connections, which muster new conditions of possibility and creative action. Resulting material assemblages mimic this kind of 
dynamics through their makeshift character, susceptible to multiple tweaks and additions meant to enhance the capacity to navigate and get hold of these emergent domains. The floating churches of Volgograd or the on-land harbour at De Ceuvel unquestionably offer some remarkable, even spectacular, instances of jury-rigged hybrids in the making. However, the novelty or inventiveness of these instances relies first and foremost on their modalities of attunement, rather than the material articulations they take. As shown in the empirical chapters, such attunements rely on creative reuse imaginaries, which enable things and affects otherwise redundant/disposed/forgotten to make surprising returns by morphing into innovative arrangements that challenge established norms and practices. The emergence of such alternative innovations is usually driven by the ingenious reworking of temporal and geographical proximities, achieved through what appear to be tinkering processes open to all kinds of transient and non-linear synergies.

\section{VII.2. Warping spatiotemporal conditions of encounter}

If the first research question tapped into the meanings innovation affords across various liminal domains, the second research question was specifically concerned with the workings of emergent alternatives. So, how do those conditions enable the emergence of alternative innovations? Whereas above I highlighted the somewhat fluid and makeshift character of amphibious sociotechnical arrangements, their rhythmic qualities are as much a function of intensive mobility as they are of transient stillness or fixity. This aspect is chiefly related to what I referred in $A$ barbour on land as the unremitting rehearsal of alternatives in generating processes of accumulation and value production. In other words, the experimental attempts to identify points of anchorage or reference that would allow those practices to exercise some degree of control over their new operative domains. As emphasized in the empirical discussion, these instances often entail unconventional synergies that warp spatiotemporal conditions of encounter, through the reworking of both temporal and geographical proximities. With the floating churches of Volgograd, we have seen how mobile religious edifices turn though their travels and mooring sites into transient organizing centers for the communities scattered along the Don, the Volga, and their tributaries. On a slightly different note, the case of De Ceuvel exposed a quaint arrangement, where houseboats and other buoyant things became part of the on-land harbour, which accommodates an experimental self-sufficient community. Subsequently, beyond beckoning the presence of alternative conditions of possibility, these idiosyncratic occurrences are also suggestive of a more generic course of action.

Driven by a logic of aspiration and differentiation, the interplay between transient instances of mobility and stillness alludes to the ways familiar arrangements are 
occasionally transposed into less-familiar contexts, as a tactic to reanimate the sediments of seemingly established orders. In this manner, resulting occurrences routinely capitalize on the productive latencies scored across those arrangements, to reveal the topological inflections that allow for alternative place-making processes to take effect. Both case studies certainly provide evidence of such inventive placemaking tactics, yet it should be taken into account that this is largely the upshot of precarious means and exclusion. Accordingly, despite their apparently radical character, the examined action repertoires in fact indicate an incremental dynamics analogous to forms of variation through mimesis. Pivoting on creative reuse imaginaries, their ethos of improvisation relies primarily on exploiting the residual surplus that stems from dominant processes of accumulation and value production. As such, new synergies are made possible, which expand the spatial and temporal reach associated with particular narratives of belonging and creative action. These are the driving force behind emergent alternatives, whether we take as a reference point the morphing between religious forms of ritual, bard music performances and sailing practices in Volgograd, or the ad hoc synergies between various creative agendas and grassroots sustainability initiatives at De Ceuvel, in Amsterdam. Perhaps most interesting to note in these cases is how complementary imaginaries balance each other in endorsing broadly inspiring narratives, which are bound to evolve in complex ecologies that thrive on normative assumptions about the meanings and workings of innovations.

\section{VII.3. The dance of contingent imaginaries and anamorphic reflections}

Related to the last point, the third research question addressed the co-emergence of innovations and their environments, labeled under the heading of expectations. This was devised as to what extent are emergent alternatives influencing incumbent political repertoires as part of the current innovation ethos? While the former research questions attained to the logic behind alternative innovations, the latter sets this against a wider background, where multiple innovation imaginaries rub against each other. Throughout the dissertation I pleaded in many occasions against normative readings of innovation, yet in what concerns the matter of intentionality, there are a few recurrent qualities that the examined cases actually share. The most immediate one pertains to the reframing of a pressing issue or of a particularly contested order, usually done in relation to the workings of formal domains. We have seen this at work with the revival of religious practices and the Cossacks' lore in Volgograd oblast. In a similar manner, the community at De Ceuvel expanded the portfolio and reach of sustainable and inclusive city-making practices in Amsterdam. The hybrid materialities informed by such attempts to expose the problematic latencies of formal domains are suggestive of another recurrent quality, indicated a bit earlier. A common feature of both cases, and 
perhaps of other initiatives alike, involves their sway in producing simple though potent narratives, which can travel fast beyond their originating microcosms. This quality is key to achieving impact in relation to incumbent innovation imaginaries.

Given the standpoint of creative reuse imaginaries as politically Other to, but inseparable from processes of creative destruction, impact is usually achieved through a rather twisted symbiosis, one of peculiar association. Thus, the last and most important recurrent quality I would like to highlight, concerns the loose/labile character of the above narratives, which enables them to contract and expand under various readings. Somewhat paradoxically, their dynamics seems to mirror that of mainstream innovations through the performative re-enactment of conditions for success. However, where these alternative narratives arguably excel is their disposition for multiple entanglements that often defy the normative distinctions between formal and informal domains. This gives rise to broad fields of resonance in recasting all sorts of anamorphic reflections across the resulting amphibious domains of contingency. In other words, the more imaginaries they interfere with or even subsume, the higher chances become for innovative spin-offs. As intimated in The Magic Mirror II, creative reuse imaginaries belong to a veritable amphibious realm of 'clay and mud', of alternating instances of sedimentation and flow, and to an ethos that comes close to what Arjen Zegwaard (2016:126) eloquently describes as a proof of 'muddy existence'. To give a final twist to the earlier remarks and the multiple mirroring instances evoked in this dissertation, I draw to a close with one of Jonty Hurwitz' artworks (figure 14), and an image that is indeed worth more than a thousand words.

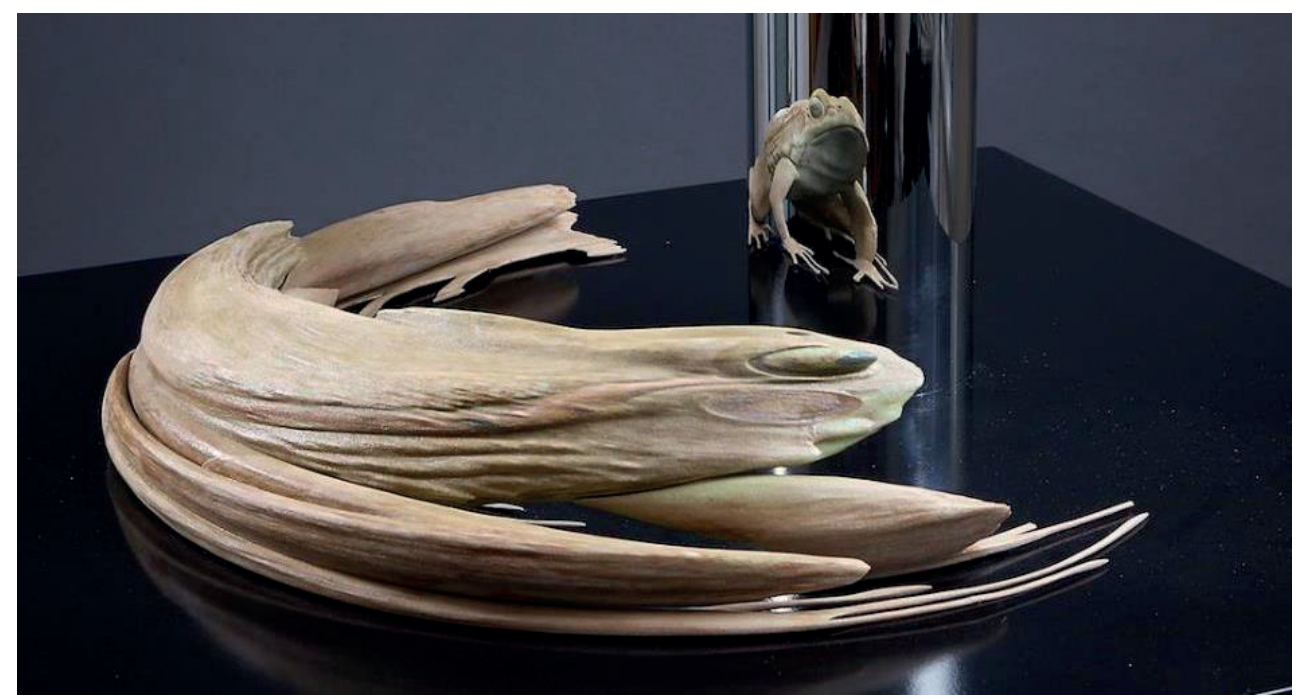

Figure 14 - Kiss of chytrid: Anamorphic frog sculpture by Jonty Hurwitz. Reproduced with kind permission of the artist. www.jontyhurwitz.com 


\section{VII.4. Coda: some tentative corollaries}

In maintaining the experimental overtone of the dissertation, this last section presents an inventory of tentative corollaries, meant not as a collection of axioms, but rather as a thought-provoking guide for those with an interest in any of the issues investigated thus far.

1. Contrary to some commonly held assumptions, creativity is a distributed and relational resource.

2. If necessity is the mother of all inventions, then mimesis is the most powerful source of innovation.

3. Innovation processes are an essential attribute of any practice.

4. To better understand the workings of alternatives, try to look for innovation in places where you least expect to find it.

5. The truly effective environments for innovation are often the most chaotic ones.

6. Innovations are indeed co-emergent with their environments, as they are co-emergent also with other innovations, which in turn are co-emergent with their environments. Try to identify the transient fixed points!

7. The surprising returns things and affects often make show how innovation processes depend as much (if not more) on past conditions as they do on future imaginaries.

8. Creative Reuse is to Creative Destruction what time travel is to Newtonian physics.

9. Even the most impressive buoyant innovations need mooring sites.

10. Topological thinking provides a salient vehicle to access realities otherwise opaque.

11. Metaphors are transgressive devices that stubbornly resist the idea of boundaries.

12. Processual philosophies are the perfect counter-referents of quantitative studies.

$$
* * *
$$



References 


\section{References}

Abrahamsson C (2012) Guest Editorial. Environment and Planning D: Society and Space 30(2): $315-321$.

Adey P (2015) Air's affinities: Geopolitics, chemical affect and the force of the elemental. Dialogues in Human Geography 5: 54-75.

Akrich M, Callon M and Latour B (2002a) The key to success in innovation part I: The art of interessement. International Journal of Innovation Management 6(2): 187-206.

Akrich M, Callon M and Latour B (2002b) The key to success in innovation part II: The art of choosing good spokespersons. International Journal of Innovation Management 6(2): 207-225.

Alexandroff P (1961) Elementary concepts of topology. New York: Courier Dover Publications.

Allen J (2011) Topological Twists: Power's Shifting Geographies. Dialogues in Human Geography 1(3): 283-98.

Allen J and Cochrane A (2010) Assemblages of State Power: Topological Shifts in the Organization of Government and Politics. Antipode 42(5): 1071-89.

Allen T J and Henn G (2006) The Organization and Architecture of Innovation: Managing the Flow of Technology. Oxford: Butterworth-Heinemann.

Amin A (2015) Animated space. Public Culture 27(2): 239-258.

Amin A (2014) Lively Infrastructure. Theory, Culture \& Society 31(7/8): 137-161.

Amin A (2007) Re-thinking the urban social. City 11(1): 100-114.

Amin A and Thrift N (2007) Cultural-economy and cities. Progress in Human Geography 31(2): 143-161.

Anderson B (2009) Affective atmospheres. Emotion, Space and Society 2(2): 77-81.

Anderson B (2006) "Transcending without transcendence": Utopianism and an Ethos of Hope. Antipode 38: 691-710.

Anderson B, Kearnes M, McFarlane C and Swanton D (2012) On assemblages and geography. Dialogues in Human Geography 2(2): 171-189.

Anderson B and Harrison P (2010) The promise of non-representational theories. In: Taking-place: non-representational theories and geography. Farnham: Ashgate, pp. 1-36.

Anderson B and Wylie J (2009) On geography and materiality. Environment and Planning A 41(2): 318-335.

Appadurai A (2013) The Future as Cultural Fact: Essays on the Global Condition. London: Verso Books. 
Archibald T (2010) The Development of Rigor in Mathematical Analysis. In: Gowers T, Barrow-Green J and Leader I (eds) The Princeton Companion to Mathematics. Princeton: Princeton University Press, pp. 117-29.

Ash J (2014) Technology and affect: Towards a theory of inorganically organised objects. Emotion, Space and Society: 1-7.

Ash J and Simpson P (2014) Geography and post-phenomenology. Progress in Human Geography: 1-19.

Asheim B, Coenen L and Vang J (2007) Face-to-face, buzz, and knowledge bases: Sociospatial implications for learning, innovation, and innovation policy. Environment and Planning C: Government and Policy 25(5): 655-670.

Van Assche K, Beunen R, Holm J and Lo M (2013) Social learning and innovation. Ice fishing communities on Lake Mille Lacs. Land Use Policy 34: 233-242.

Bachelard G (1994) The Poetics of Space. Boston: Beacon Press.

Badiou A (2006) Mathematics and Philosophy. In: Duffy S (ed.) Virtual mathematics: the logic of difference. Manchester: Clinamen Press, pp.12-30.

Barba Lata I and Minca C (2016) The surface and the abyss/Rethinking topology. Environment and Planning D: Society and Space 34(3): 438-455.

Barba Lata I (2015) Topology and object formation. In: Beunen R, Van Assche K and Duineveld M (eds) Evolutionary Governance Theory: Theory and Applications. Cham: Springer, pp. $155-165$.

Barba Lata I (2014) Review - Rob Shields' Spatial Questions: Cultural Topologies and Social Spatialisations. Society \& Space Open Site.

Barber S and Hale M (2012) Enacting the Never-Was: Upcycling the Past, Present and Future in Steampunk. In: Taddeo J A and Miller C J (eds) Steaming into a Victorian Future: A Steampunk Anthology, Lanham: Scarecrow Press, pp. 165-183.

Bathelt H, Malmberg A and Maskell P (2004) Clusters and knowledge: local buzz, global pipelines and the process of knowledge creation. Progress in Human Geography 28(1): 31-56.

Beardon A F (2010) Riemann Surfaces. In: Gowers T, Barrow-Green J and Leader I (eds) The Princeton Companion to Mathematics. Princeton: Princeton University Press, pp. 282-83.

Benjamin W (2002) The Arcades Project .R. Tiedemann (ed.). Cambridge: Harvard University Press.

Bennett J (2010) Vibrant Matter: A Political Ecology of Things. Durham: Duke University Press. 
Bergelson V (2010) Fixed Point Theorems. In: Gowers T, Barrow-Green J and Leader I (eds) The Princeton Companion to Mathematics. Princeton: Princeton University Press, pp. 693-696.

Blum V and Secor A (2011) Psychotopologies: closing the circuit between psychic and material space. Environment and Planning D: Society and Space 29: 1030-1048.

Boyle M (2011) Commentary. The New Urban Politics Thesis: Ruminations on MacLeod and Jones' Six Analytical Pathways. Urban Studies 48(12): 2673-2685.

Brook P (1996) The Empty Space: A Book About the Theatre: Deadly, Holy, Rough, Immediate. New York: Simon and Schuster.

Brown G, Kraftl P, Pickerill J, Upton C (2012) Holding the future together: Towards a theorisation of the spaces and times of transition. Environment and Planning A 44(7): 1607-1623.

Bulkeley H, Edwards G and Fuller S (2014) Contesting climate justice in the city: Examining politics and practice in urban climate change experiments. Global Environmental Change 25(1): 31-40.

Bulkeley H and Castán Broto V (2013) Government by experiment? Global cities and the governing of climate change. Transactions of the Institute of British Geographers 38(3): 361-375.

Cabanne P and Duchamp M (1987) Dialogues with Marcel Duchamp, London: Da Capo Press.

Cadman L (2009) Nonrepresentational theory/nonrepresentational geographies. International encyclopaedia of human geography, pp.1-8.

Calderbank M (2003) Surreal Dreamscapes: Walter Benjamin and the Arcades. Papers of Surrealism, (1): 1-13.

Callon M (2007) What Does It Mean to Say That Economics Is Performative? In: MacKenzie D, Muniesa F and Siu L (eds) Do Economists Make Markets? On the Performativity of Economics. Princeton: Princeton University Press, pp. 311-356.

Callon M and Law J (2004) Guest editorial. Environment and Planning D: Society and Space 22(1): 3-12.

Calzada I and Cobo C (2015) Unplugging: Deconstructing the Smart City. Journal of Urban Technology 22(1): 23-43.

Carroll L (1920) Alice's Adventures in Wonderland. New York: Macmillan.

de Certeau M (1984) The Practice of Everyday Life. Berkley: University of California Press.

Chaloupek G (1995) Long-term economic perspectives compared: Joseph Schumpeter and Werner Sombart. The European Journal of the History of Economic Thought 2(1): 127149.

Châtelet G (2000) Figuring space: philosophy, mathematics, and physics. Dordrecht: Kluwer. 
Chesbrough H W (2003) Open Innovation: The New Imperative for Creating and Profiting from Technology. Boston: Harvard Business School Press.

Coleman M (2011) Topologies of practice. Dialogues in Human Geography 1(3): 308-311.

Collier S J (2009) Topologies of Power Foucault's Analysis of Political Government. Theory, Culture \&o Society 26(6): 78-108.

Corry L (2010) The Development of the Idea of Proof. In: Gowers T, Barrow-Green J and Leader I (eds) The Princeton Companion to Mathematics. Princeton: Princeton University Press, pp. 129-42.

Cowen T (2009) Creative Destruction: How Globalization Is Changing the World's Cultures. Princeton: Princeton University Press.

Crang M (2012) Temporal ecologies: Multiple times, multiple spaces, and complicating space times. Environment and Planning A 44: 2119-2123.

Crang M (2010) The death of great ships: Photography, politics, and waste in the global imaginary Environment and Planning A 42: 1084-1102.

Crang M and Travlou P S (2001) The city and topologies of memory. Environment and Planning D: Society and Space 19(2): 161-177.

Cresswell T (2012) Value, gleaning and the archive at Maxwell Street, Chicago. Transactions of the Institute of British Geographers 37: 164-176.

Cresswell T (2012) Mobilities II: Still. Progress in Human Geography 36(5): 645-653.

Cresswell T (2010) Towards a Politics of Mobility.Environment and Planning D: Society and Space 28(1): 17-31.

Cresswell T and Martin C (2012) On Turbulence: Entanglements of Disorder and Order on a Devon Beach. Tijdschrift voor Economische en Sociale Geografie 103: 516-529.

Davies G (2010) Where do experiments end? Geoforum 41(5): 667-670.

Davis N (2003) A Long Walk to Church: A Contemporary History of Russian Orthodoxy, Boulder: Westview Press.

Davies A and Mullin S (2012) Waste and community enterprises in Ireland: Co-operation, collaboration and the formation of the community resource network. In: Davies A (ed.) Enterprising communities: Grassroots Sustainability Innovations. Bingley: Emerald, pp. 49-64.

de Risi V (2007) Geometry and Monadology: Leibniz's Analysis Situs and Philosophy of Space Basel: Springer Science \& Business Media.

DeLyser D (2015) Collecting, kitsch and the intimate geographies of social memory: a story of archival autoethnography. Transactions of the Institute of British Geographers 40:209-222.

Deleuze G (2006) The Fold. London: Continuum. 
Deleuze G (1994) Difference and Repetition. New York: Columbia University Press.

Deleuze G and Guattari F (2000) Anti-Oedipus. Minneapolis: University of Minnesota Press.

Deleuze G and Guattari F (1987) A Thousand Plateaus: Capitalism and Schizophrenia.

Minneapolis: University of Minnesota Press.

Della Dora V (2011) Circulating Sacred Place: Fin-de-Siècle Russian Cards of Mount Athos as Travelling Object-Icons. In: Brace C, Bailey A, Carter S, Harvey D and Thomas N (eds) Emerging Geographies of Belief, Cambridge Scholars Publishing, pp. $168-192$.

Della Dora V and Sooväli-Sepping H (2009) Sacred Space and Uncomfortable Memories: The Alexander Nevski Russian Orthodox Cathedral in Tallinn, Estonia. In: Leveque P L and Ruiz del Arbol M (eds) Heritage, Images, Memory of European Landscapes. L'Harmattan, pp. 215 - 229.

Dieudonné J (2011) Preface to the 1977 Edition, In: Lautman A (ed.) Mathematics, Ideas and the Physical Real. Trans. S Duffy. London, pp. xxxix-xlii.

Dixon D P and Jones J P (2015) The tactile topologies of Contagion. Transactions of the Institute of British Geographers 40: 223-234.

Durie R (2006) Problems in the relation between maths and philosophy. In: Duffy S (ed.) Virtual mathematics: the logic of difference. Manchester: Clinamen Press, pp. 169-186.

Dwyer C, Gilbert D and Shah B (2013) Faith and suburbia: Secularisation, Modernity and the changing geographies of religion in London's suburbs. Transactions of the Institute of British Geographers 38: 403-419.

Dwyer C and Davies G (2010) Qualitative methods III: animating archives, artful interventions and online environments. Progress in Human Geography 34(1): 88-97.

Eco U (2005) Innovation \& Repetition: Between Modern \& Postmodern Aesthetics. Daedalus 134(4): 191-207.

Elden S (2011) What's Shifting? Dialogues in Human Geography 1(3): 304-7.

Evans J P (2011) Resilience, ecology and adaptation in the experimental city. Transactions of the Institute of British Geographers 36(2): 223-237.

Fall J J and Minca C (2013) Not a geography of what doesn't exist, but a countergeography of what does.Rereading Giuseppe Dematteis' Le Metafore della Terra. Progress in Human Geography 37(4): 542-563.

Faulconbridge J (2013) Mobile "green" design knowledge: Institutions, bricolage and the relational production of embedded sustainable building designs. Transactions of the Institute of British Geographers 38(2): 339-353. 
Ferreirós J (2010a) The Crisis in the Foundations of Mathematics. In: Gowers T, BarrowGreen J and Leader I (eds) The Princeton Companion to Mathematics. Princeton: Princeton University Press, pp. 142-156.

Ferreirós J (2010b) Jules Henri Poincaré. In: Gowers T, Barrow-Green J and Leader I (eds) The Princeton Companion to Mathematics. Princeton: Princeton University Press, pp.785-787.

Florida R (2009) Who's Your City?: How the Creative Economy is Making where to Live the Most Important Decision of Your Life. New York: Basic Books.

Fredriksen A (2014) Emergency shelter topologies: locating humanitarian space in mobile and material practice. Environment and Planning D: Society and Space 31: 147-162.

Froese P (2004) Forced Secularization in Soviet Russia: Why an Atheistic Monopoly Failed. Journal for the Scientific Study of Religion 43: 35-50.

Fuller M and Goffey A (2012) Digital Infrastructures and the Machinery of Topological Abstraction. Theory, Culture \& Society 29(4/5): 311-333.

Fuller R B and Applewhite E J (1979) Synergetics: Explorations in the Geometry of Thinking. Macmillan.

Fuller R B (1969) Operating manual for spaceship earth. New York: Simon and Schuster.

Garud R, Gehman J and Kumaraswamy A (2011) Complexity arrangements for sustained innovation: Lessons from 3M Corporation. Organization Studies 32: 737-767.

Ghertner D A (2008) Analysis of New Legal Discourse behind Delhi's slum demolitions. Economic \& Political Weekly 43(20): 57-66.

Giaccaria P and Minca C (2011) Topographies/topologies of the Camp: Auschwitz as a Spatial Threshold. Political Geography 30(1): 3-12.

Gieryn T F (2008) Laboratory Design for Post-Fordist Science. Isis 99(4): 796-802.

Girard R (1990) Innovation and repetition. SubStance 19(2): 7-20.

Godin B and Lucier P (2014) Innovo: On the Vicissitudes and Variations of a Concept. Project on the Intellectual History of Innovation: Working Paper No. 19: 1-36.

Godin B and Lucier P (2012) Innovation and Conceptual Innovation in Ancient Greece. Project on the Intellectual History of Innovation: Working Paper No. 12: 1-31.

Gray J (2010a) Geometry. In: Gowers T, Barrow-Green J and Leader I (eds) The Princeton Companion to Mathematics. Princeton: Princeton University Press, pp. 83-95.

Gray J (2010b) Fuchsian Groups. In: Gowers T, Barrow-Green J and Leader I (eds) The Princeton Companion to Mathematics. Princeton: Princeton University Press, pp.208-210.

Gray J (2010c) Georg Friedrich Bernhard Riemann. In: Gowers T, Barrow-Green J and Leader I (eds) The Princeton Companion to Mathematics. Princeton: Princeton University Press, pp. 774-776. 
Gray J (2010d) Élie Joseph Cartan. In: Gowers T, Barrow-Green J and Leader I (eds) The Princeton Companion to Mathematics. Princeton: Princeton University Press, p. 794.

Greenfield A (2013) Against the Smart City. New York: Do Projects.

Gregson N, Crang M, Ahamed F, Akhter N and Ferdous R (2010) Following things of rubbish value: End-of-life ships, "chock-chocky" furniture and the Bangladeshi middle class consumer. Geoforum 41: 846-854.

Hannah M (2006) Torture and the Ticking Bomb: The War on Terrorism as a Geographical Imagination of Power/Knowledge. Annals of the Association of American Geographers 96(3): 622-640.

Hardt M (2010) Two Faces of Apocalypse : A Letter from Copenhagen. Polygraph 22: 1726.

Harvey D (1997) Justice, Nature and the Geography of Difference. Wiley-Blackwell, UK.

Henderson L D (2009) The Image and Imagination of the Fourth Dimension in Twentieth-Century Art and Culture. Configurations 17: 131-160.

Hilbert D and Cohn-Vossen S (1990) Geometry and the imagination. New York: Chelsea Publishing.

Hill L J (2015) Human geography and archaeology: Strange bedfellows? Progress in Human Geography 39(4): 412-431.

Hinchliffe S and Whatmore S (2006) Living cities: Towards a politics of conviviality. Science as Culture 15(2): 123-138.

von Hippel E (2005) Democratising Innovation. Cambridge: MA: MIT Press.

Holloway J (2003) Make-believe: spiritual practice, embodiment, and sacred space. Environment and Planning A 35: 1961-1974.

Holquist P (1997) "Conduct Merciless Mass Terror": Decossackization on the Don, 1919. Cahiers du Monde russe 38: 127-162.

Ingold T (2007) Materials against materiality. Archaeological Dialogues. 14: 1-16.

Ivanov S and Suprun V (2006) Floating temples of Russia, The naval history of clergy [Plavuchiye khramy Rossii. Istoriya flotskogo dukhovenstva], Volgograd: VGIPK RO.

Jacobs J M and Merriman P (2011) Practising architectures. Social \& Cultural Geography 12(3): 211-222.

Jeanes E L (2006) Resisting Creativity, Creating the New. A Deleuzian Perspective on Creativity. Creativity and inovation Management 15(2): 127-134.

Jensen J S, Hagelskjær Lauridsen E, Farné Fratini C and Hoffman B (2015) Harbour bathing and the urban transition of water in Copenhagen: Junctions, mediators, and urban navigations. Environment and Planning A 47(3): 554-570. 
Jones M (2014) Kapoorian geographies of relationality: The baroque, topological twists, phase space in action. Environment and Planning A 46(11): 2585-2603.

Jones M (2009) Phase Space: Geography, Relational Thinking, and beyond. Progress in Human Geography 33(4): 487-506.

Keighren I M, Abrahamsson C and della Dora V (2012) On canonical geographies. Dialogues in Human Geography 2(3): 296-312.

Kleiner I (2010) Karl Weierstrass. In: Gowers T, Barrow-Green J and Leader I (eds) The Princeton Companion to Mathematics. Princeton: Princeton University Press, pp. 770 771.

Knobloch E (2010) Gottfried Wilhelm Leibniz. In: Gowers T, Barrow-Green J and Leader I (eds) The Princeton Companion to Mathematics. Princeton: Princeton University Press, pp. 743-745.

Kurz H D (2016) Adam Smith on markets, competition and violations of natural liberty. Cambridge Journal of Economics 40(2): 615-638.

Kwinter S (2007) Far from Equilibrium: Essays on Technology and Design Culture. Davidson C (ed.). Barcelona: Actar-D.

Lancione M (2016) The assemblage of life at the margins. In: Lancione M (ed.) Rethinking Life at the Margins: The Assemblage of Contexts, Subjects, and Politics. London: Routledge, pp. 3-26.

Latham A (2011) Topologies and the multiplicities of space-time. Dialogues in Human Geography 1(3): 312-315.

Latham A (2002) Retheorizing Scale of Globalization: Topologies, Actor-Networks and Cosmopolitanism. In: Herod A and Wright M W (eds) Geographies of power: placing scale. Blackwell Publishing, UK, pp. 115-44.

Latham A and McCormack D (2004) Moving cities: rethinking the materialities of human geographies. Progress in Human Geography 28(6): 701-724.

Latour B (1999) Pandora's Hope: Essays on the Reality of Science Studies. Cambridge: Harvard University Press.

Latour B (1993) We have never been modern. Cambridge, MA: Harvard University.

Lautman A (2011) Mathematics, Ideas and the Physical Real. Trans. S Duffy. London: Continuum.

Law J (2004) After method: mess in social science research. London: Routledge.

Law J (2002) Objects and Spaces. Theory, Culture \& Society 19(5/6): 91-105.

Law J and Mol A (2001) Situating technoscience: an inquiry into spatialities. Environment and Planning D: Society and Space 19: 609-622. 
Lazarski C (1992) Vladimir Vysotsky and His Cult. Russian Review 51: 58-71

Lefaivre L (2002) Space, place and play. In: Lefaivre L and de Roode I (eds) Aldo van Eyck: the playgrounds and the city. Rotterdam: NAI Publishers, pp. 16-58.

Lenoir T and Alt C (2002) Flow, Process, Fold: Intersections in Bioinformatics and Contemporary Architecture. In: Picon A and Ponte A (eds) Science, Metaphor, and Architecture. Princeton: Princeton Architectural Press, pp. 314-353.

Lorenc T (2012) Afterword: Tarde's Pansocial Ontology. In: Tarde G Monadology and Sociology. Lorenc T (ed. and trans.) Melbourne: Re.Press, pp. 71-95.

Lorimer H (2007) Cultural Geography: Worldly Shapes, Differently Arranged. Progress in Human Geography 31(1): 89-100.

Lorimer H (2007) Songs from before - shaping the conditions for appreciative listening. In: Gagen E, Lorimer H and Vasudevan A (eds) Practising the archive: reflections on method and practice in historical geography. London: Royal Geographical Society (Historical Geography Research Group Series; No. 40), pp. 57-73.

Luque-Ayala A and Marvin S (2015) Developing a critical understanding of smart urbanism? Urban Studies 52(12): 2105-2116.

Luque A, Mcfarlane C and Marvin S (2014) Smart urbanism: Cities, grids and alternatives? In: Hodson M and Simon M (eds) After Sustainable Cities? London: Routledge, pp. 74-90.

Lury C, Parisi L and Terranova T (2012) Introduction: The Becoming Topological of Culture. Theory, Culture \& Society 29(4-5): 3-35.

MacLeod G and Jones M (2011) Renewing Urban Politics. Urban Studies 48(12): 24432472.

Macleod G and Jones M (2007) Territorial, Scalar, Networked, Connected: In What Sense a 'Regional World'?. Regional Studies 41(9): 1177-1191.

Majid S (2012) On Space and Time. New York: Cambridge University Press.

Malpas J (2012) Putting Space in Place: Philosophical Topography and Relational Geography. Environment and Planning D: Society and Space 30(2): 226-242.

Marques I d C (2004) Mathematical metaphors and politics of presence/absence. Environment and Planning D: Society and Space 22: 71-82.

Martin L and Secor A J (2013) Towards a post-mathematical topology. Progress in Human Geography 38(3): 420-438.

Massey D (2005) For Space. London: Sage Publications.

Massey D (1999) Space-Time, 'Science' and the Relationship between Physical Geography and Human Geography. Transactions of the Institute of British Geographers 24(1995): 261-276. 
Massey D (1995) Places and Their Pasts. History Workshop Journal 1(39): 182-192.

McCann E (2011) Urban Policy Mobilities and Global Circuits of Knowledge: Toward a Research Agenda. Annals of the Association of American Geographers: 101(1): 107-130.

McCraw T K (2007) Prophet of Innovation: Joseph Schumpeter and Creative Destruction. Cambridge: Harvard University Press.

McCormack D P (2014) Atmospheric things and circumstantial excursions. Cultural Geographies 21: 605-625.

Mcfarlane C (2015) The Geographies of Urban Density : Topology, Politics and the City. Progress in Human Geography: 1-20.

McFarlane C (2012) The Entrepreneurial Slum: Civil Society, Mobility and the Coproduction of Urban Development. Urban Studies 49(13): 2795-2816.

McFarlane C (2011a) The city as a machine for learning. Transactions of the Institute of British Geographers 36(3): 360-376.

McFarlane C (2011b) The city as assemblage: Dwelling and urban space. Environment and Planning D: Society and Space 29(4): 649-671.

Mcfarlane C and Vasudevan A (2014) Informal infrastructures. In: Adey P, Bissell D, Hannam K, Merriman P and Sheller M (eds) The Routledge Handbook of Mobilities. London: Routledge, pp. 256-264.

Merriman P (2015) Mobilities II: Cruising. Progress in Human Geography: 1-10.

Mezzadra S and Neilson B (2012) Between Inclusion and Exclusion: On the Topology of Global Space and Borders. Theory, Culture \& Society 29(4/5): 58-75.

Michael M and Rosengarten M (2012) HIV, Globalization and Topology: Of Prepositions and Propositions. Theory, Culture \& Society 29(4/5): 93-115.

Michaelides P G, Milios J G, Vouldis A and Lapatsioras S (2010) Heterodox influences on Schumpeter. International Journal of Social Economics 37(3): 197-213.

Miller D (2011) The power of making. In: Charny D (ed.) The Power of Making. London: V\&A Publishing, pp. 14-27.

Mol A (2002) The Body Multiple: Ontology in Medical Practice. Durham: Duke University Press.

Mol A and Law J (1994) Regions, Networks and Fluids: Anaemia and Social Topology. Social Studies of Science 24(4): 641-671.

Moberly C H (1862) An account of the conveyance of light-draught steamers from England to the River Volga, with remarks on the inland navigation of Russia, Dublin: Transactions of the Institution of Civil Engineers 7(1): 1-16.

Morales M d C, Harris L and Öberg G (2014) Citizenshit: The right to flush and the urban sanitation imaginary. Environment and Planning A 46(12): 2816-2833. 
Moreira T (2004) Surgical monads: a social topology of the operating room. Environment and Planning D: Society and Space 22: 53-70.

Moore M (2011) Marcel Duchamp: "Twisting Memory for the Fun of It" or a Form of Retroactive Interference? - Recalling the Impacts of Leaving Home on the Readymade. Memory Connection 1: 393-403.

Moore S A (2012) Garbage matters: Concepts in new geographies of waste. Progress in Human Geography, 36(6): 780-799.

Morton F (2005) Performing ethnography: Irish traditional music sessions and new methodological spaces. Social \& Cultural Geography 6(5): 661-676.

Murdoch J (2006) Post-structuralist Geography: A Guide to Relational Space. London: Sage Publications.

Netz R (2010) What did Greek mathematicians find beautiful? Classical Philology 105(4): 426-444.

Netz R (2010) Barbed Wire: An Ecology of Modernity. Middletown: Wesleyan University Press.

Netz R (2003) The Shaping of Deduction in Greek Mathematics: A Study in Cognitive History, Cambridge, UK: Cambridge University Press.

Nietzsche, F W (1979) Philosophy and truth: selections from Nietssche's notebooks of the early 1870 's, Breazeale D (ed.), Humanities Press.

Nieuwenhuis C and Debord G (1958) La déclaration d'Amsterdam. Internationale situationniste, (2): 31-32.

Oliphant L (1854) The Russian Shores of the Black Sea in the Autumn of 1852: With a Voyage Down the Volga, and a Tour Through the Country of the Don Cossacks. Edinburgh: W. Blackwood and Sons.

Olson L (2004) Performing Russia: Folk Revival and Russian Identity. London: Routledge.

Olsson G (2007) Abysmal: A Critique of Cartographic Reason. Chicago: The University of Chicago Press.

Olsson G (1982) “-/-". In Gould P and Olsson G (eds) A Search for Common Ground. London: Pion, pp. 223-231.

Oudenampsen M (2010) Aldo van Eyck and the City as Play-ground. In Méndez de Andés A (ed.) Urbanacción 07/09. Madrid: La Casa Encendida, pp. 25-39.

Owens L (2009) Cracking Under Pressure: Narrating the Decline of the Amsterdam Squatters' Movement. Amsterdam: Amsterdam University Press.

Paasi A (2011a) Geography, Space and the Re-Emergence of Topological Thinking. Dialogues in Human Geography 1(3): 299-303. 
Paasi A (2011b) From region to space part II. In: Agnew J A and Duncan J S (eds) The Wiley-Blackwell Companion to Human Geography. Chichester: Wiley-Blackwell, pp. 161175.

Peck J (2005) Struggling with the Rise of the Creative Class. International Journal of Urban and Regional Research 29(4): 740-770.

Perez C (1983) Structural change and assimilation of new technologies in the economic and social systems. Futures 15(5): 357-375.

Phillips J W (2013) On Topology. Theory, Culture \& Society 30(5): 122-52.

Pickerill J and Maxey L (2009) Geographies of sustainability: Low impact developments and radical spaces of innovation. Geography Compass 3(4): 1515-1539.

Pickerill J and Chatterton P (2006) Notes towards autonomous geographies: creation, resistance and self management as survival tactics. Progress in Human Geography 30(6): 730-746.

Plotnitsky A (2012) Experimenting with ontologies: Sets, spaces, and topoi with Badiou and Grothendieck. Environment and Planning D: Society and Space 30(2): 351-368.

Plotnitsky A (2009) Bernhard Riemann's Conceptual Mathematics and the Idea of Space. Configurations (17): 105-130.

Plotnitsky A (2006) Manifolds: On the Concept of Space in Riemann and Deleuze. In: Duffy S (ed.) Virtual mathematics: the logic of difference. Manchester: Clinamen Press, pp. 187-208.

Plotnitsky A (2002) The Knowable and the Unknowable: Modern Science, Nonclassical Thought, and the "Two Cultures". The University of Michigan Press.

Poincaré H (2010) Papers on Topology: Analysis Situs and Its Five Supplements. American Mathematical Society.

Pop-Up City (2015) How a hip area in Amsterdam blossoms out of nothing. Smart magazine (07/14/2015):http://www.smart-magazine.com/en/de-ceuvel-amsterdamurban-project/

Pruijt H (2004) Squatters in the Creative City: Rejoinder to Justus Uitermark. International Journal of Urban and Regional Research 28(3): 699-705.

Purcell M (2008) Recapturing democracy: Neoliberalization and the struggle for alternative urban futures. New York: Routledge.

Rabinow P (2008) Marking time: on the anthropology of the contemporary. Princeton: Princeton University Press.

Rantisi N M and Leslie D (2010) Materiality and creative production: The case of the Mile End neighborhood in Montréal. Environment and Planning A 42(12): 2824-2841.

Ray M A (1997) Pamphlet Architecture 20: Seven Partly Underground Rooms and Buildings for Water, Ice, and Midgets. New York: Princeton Architectural Press. 
Reinert E S (2002) Schumpeter in the context of two canons of economic thought. Industry and Innovation 9(1-2): 23-39.

Reinert H and Reinert E S (2006) Creative Destruction in Economics: Nietzsche, Sombart, Schumpeter. In: Backhaus J G and Drechsler W (eds) Friedrich Nietzsche (1844--1900): Economy and Society. Boston, MA: Springer US, pp. 55-85.

Richardson L, Purdam K, Cotterill S, Rees J, Squires G and Askew R (2014) Responsible citizens and accountable service providers? Renegotiating the contract between citizen and state. Environment and Planning A 46(7): 1716-1731.

Riemann B (1882) On the Hypotheses Which Lie at the Bases of Geometry. In: Clifford W K, Tucker R and Smith H J S (eds) Mathematical Papers. Trans. WK Clifford. London: Macmillan and Co., pp. 55-71.

Rotman B (2012) Topology, Algebra, Diagrams. Theory, Culture \& Society 29(4-5): 247260.

Rowe N (1870) A Journey on the Volga. In Bates H W (ed.) Illustrated travels: a record of discovery, geography and adventure, New York: Cassell, Petter \& Galpin, pp. 22-32.

Roy A (2009) Why India cannot plan its cities: informality, insurgence and the idiom of urbanization. Planning Theory 8(1): 76-87.

Ruttan V W (1959) Usher and Schumpeter on Invention, Innovation, and Technological Change. The Quarterly Journal of Economics 73(4): 596-606.

Sachs H, Stiebitz M and Wilson R J (1988) An Historical Note: Euler's Königsberg Letters. Journal of Graph Theory 12(1): 133-39.

Salazar Sutil N (2013) Rudolf Laban and Topological Movement: A Videographic Analysis. Space and Culture 16(2): 173-193.

Sandifer E (2010) Leonhard Euler. In: Gowers T, Barrow-Green J and Leader I (eds) The Princeton Companion to Mathematics. Princeton: Princeton University Press, pp. 747749.

Santner E L (2006) On Creaturely Life: Rilke, Benjamin, Sebald. Chicago: University of Chicago Press.

Schumpeter J A (1975) Capitalism, Socialism, and Democracy, New York: Harper \& Row.

Schuetze C F (2014) Building a Community on Polluted Land - Ex-Shipyard in Amsterdam Houses Shops and Offices. The New York Times (11/19/2014)

Scott A J (1999) The cultural economy: geography and the creative field. Media, Culture \& Society 21(6): 807-817.

Scriven R (2014) Geographies of Pilgrimage: Meaningful Movements and Embodied Mobilities. Geography Compass 8(4):249-261.

Serres M (2008) The Five Senses: A Philosophy of Mingled Bodies. London: Continuum.

Serres M (2006) The Troubadour of Knowledge. The University of Michigan Press. 
Serres M (2000) The Birth of Physics, Manchester: Clinamen Press.

Serres M (1997) Genesis. Trans. James G and Nielson J. The University of Michigan Press.

Serres M and Adkins T (2012) Differences: chaos in the history of the sciences. Environment and Planning D: Society and Space 30(2): 369-380.

Serres M and Latour B (1995) Conversations on Science, Culture, and Time. University of Michigan Press.

Seyfang G and Smith A (2007) Grassroots Innovations for sustainable development: Towards a new research and policy agenda. Environmental Politics 16(4): 584-603.

Sheller M (2004) Mobile publics: beyond the network perspective. Environment and Planning D: Society and Space 22(1): 39-52.

Sheppard E (2002) The Spaces and Times of Globalization: Place, Scale, Networks, and Positionality. Economic Geography 78(3): 307-330.

Shields R (2013) Spatial Questions: Cultural Topologies and Social Spatialisations. London: Sage Publications.

Sidorov D (2000) Playing Chess with Churches: Russian Orthodoxy as Re(li)gion. Historical Geography 28: 208-233.

Simone A (2013) Cities of Uncertainty: Jakarta, the Urban Majority, and Inventive Political Technologies. Theory, Culture \& Society 30(7-8): 243-263.

Simone A (2010) City Life from Jakarta to Dakar: Movements at the Crossroads. New York: Routledge.

Simone A (2008) Emergency Democracy and the "Governing Composite". Social Text 95 26(2):13-34.

Skinner B (1994) Identity formation in the Russian Cossack revival. Europe-Asia Studies 46: 1017-1037.

Smith R G (2003) World city topologies. Progress in Human Geography 27(5): 561-582.

Smyth J V (1997) A Glance at SunSet: Numerical Fundaments in Frege, Wittgenstein, Shakespeare, Beckett. In: Smith B H and Plotnitsky A (eds) Mathematics, Science, and Postclassical Theory. Durham: Duke University Press, pp. 212-242.

Soja E W (2002) On Spuistraat: The Contested Streetscape in Amsterdam. In: Borden I, Kerr J and Rendell J (eds) The Unknown City: Contesting Architecture and Social Space. Cambridge: MIT Press, pp. 280-295.

Soja E W (2009) The city and spatial justice. Conference Spatial Justice, Nanterre, Paris, March 2008.

Steinberg P and Peters K (2015) Wet ontologies, fluid spaces: Giving depth to volume through oceanic thinking. Environment and Planning D: Society and Space 33(2): 247-264. 
Stewart K (2011) Atmospheric attunements. Environment and Planning D: Society and Space 29: 445-453.

Stigt W Van (1979) The rejected parts of Brouwer's dissertation on the foundations of mathematics. Historia mathematica 6(1979): 385-404.

Strathern M (1992) Reproducing the Future: Essays on Anthropology, Kinship and the New Reproductive Technologies. Manchester: Manchester University Press.

Stubhaug A (2010) Camille Jordan. In: Gowers T, Barrow-Green J and Leader I (eds) The Princeton Companion to Mathematics. Princeton: Princeton University Press, pp. 777778.

Sundaram R (2010) Pirate Modernity: Delhi's Media Urbanism. London: Routledge.

Talygin A (2011) Handbook of passenger ships of the Volga basin (1885-1914) [Spravochnik po passazhirskim sudam basseyna Volgi (1885-1914 gody)]:http://oldriver.ru/ships/.

Tarde G (2012) Monadology and Sociology. Lorenc T (ed. and trans.). Melbourne: Re.Press.

Tarde G (1903) The Laws of Imitation. New York: Henry Holt and Company.

Thom R (1989) Structural Stability and Morphogenesis. Boulder: Westview Press.

Thompson D W (1945) On Growth and Form. Cambridge: Cambridge University Press.

Thrift N (2008) Non-representational Theory: Space, Politics, Affect, London: Routledge.

Thrift N (2006) Re-inventing invention: new tendencies in capitalist commodification. Economy and Society 35(2): 279-306.

Thrift N (2006) Space. Theory, Culture \& Society 23(2-3): 139-46.

Thrift N (1996) Spatial Formations. London: Sage Publications.

Topol E J (2012) The Creative Destruction of Medicine: How the Digital Revolution Will Create Better Health Care. New York: Basic Books.

Totaro B (2010) Algebraic Topology. In: Gowers T, Barrow-Green J and Leader I (eds) The Princeton Companion to Mathematics. Princeton: Princeton University Press, pp. 383-396.

Tse J K H (2014) Grounded theologies: "Religion" and the "secular" in human geography. Progress in Human Geography 38: 201-220.

Tsing A L (2015) The Mushroom at the End of the World: On the Possibility of Life in Capitalist Ruins. Princeton: Princeton University Press.

Tuan Y-F (1989) Surface Phenomena and Aesthetic Experience. Annals of the Association of American Geographers 79(2): 233-241.

Urry J (2005) The Complexities of the Global. Theory, Culture \& Society 22(5): 235-254. 
Usov A (1993) Floating Temple, Formerly "Pirate"[Plavuchikh khram, byvshiy "Pirat"]. In: Naval [Naval] 2: 38-44.

Van Dalen D (2010) Luitzen Egbertus Jan Brouwer. In: Gowers T, Barrow-Green J and Leader I (eds) The Princeton Companion to Mathematics. Princeton: Princeton University Press, pp.799-800.

Vasantkumar C (2013) The scale of scatter: rethinking social topologies via the anthropology of 'residual' China. Environment and Planning D: Society and Space 31(5): 918-934.

Vasudevan A (2015a) The autonomous city: Towards a critical geography of occupation. Progress in Human Geography 39(3): 316-337.

Vasudevan A (2015b) The makeshift city: Towards a global geography of squatting. Progress in Human Geography 39(3): 338-359.

Viney W (2014) Waste: A Philosophy of Things. London: Bloomsbury Publishing.

Weyl H (2010) The Concept of a Riemann Surface. Trans. GR MacLane. Snowball Publishing.

Whatmore S (2003) Generating materials. In: Pryke M, Rose G and Whatmore S (eds) Using Social Theory: Thinking Through Research. London: Sage Publications, pp. 89-104.

Whitehead A N (1967) Adventures of Ideas, New York: Simon and Schuster.

Whitehead N L (2009) Post-Human Anthropology. Identities: Global Studies in Culture and Power 16(1): 1-32.

Wylie J (2005) A single day's walking: Narrating self and landscape on the South West Coast Path. Transactions of the Institute of British Geographers 30(2): 234-247.

Zegwaard A and Wester P (2014) Inside matters of facts: Reopening dams and debates in the Netherlands. Water Alternatives 7(3): 464-479.

Zegwaard A (2016) MUD: Deltas dealing with uncertainties. Vrije Universiteit Amsterdam. 

Summary 


\section{Summary}

This dissertation dwells on an experimental approach to the emergence of alternative innovations, interrogated through their spatiotemporal and material conditions. Proceeding from the more recent spate of contributions that grant recognition to innovation processes as a common feature of any practice, this research seeks to expand the understanding of innovation beyond canonical interpretations of the subject matter. This opens up a bewildering matrix of potentialities to tackle the emergence of alternatives, often to be recovered from the very dynamics of mainstream innovations that branch out beyond their original purpose. Moreover, the contingent character of mainstream and alternative innovations connotes processes of varying dynamics and rhythmic qualities, which appear to escape the sole grip of linear or cyclical interpretations. Instructed by this preliminary set of assumptions, this investigation belongs to an amphibious domain of enquiry, one that takes shape at the interface between presumably grounded and more fluid readings of innovation processes. Aligned to the amphibious conceptual imaginary, there is also the thematic repertoire and empirical ambit of case studies explored within the dissertation. As such, the evoked conceptual liminality dictated the particular focus on amphibious practices, as the referents of material and affective dispositions, as well as of narratives of belonging scored across land-water interfaces.

The main case studies presented in chapters IV and $\mathrm{V}$ were the result of an exploratory phase, with its point of departure in a pilot study conducted on the emergence of floating urbanization solutions in the Netherlands. The surveyed modalities of inhabiting land-water interfaces led me to wonder on the existence of alternative conditions of possibility to what otherwise appeared and were also tagged as very innovative attempts to reimagine urban dwelling. This struck me as a thorny task: where do you start in qualifying something as innovative or not? It took another survey of historical practices and some lengthy reflection sessions to realize that beyond the shifts and turns it has supposedly informed, innovation is much more performative than I initially thought. Thus, I started conducting ethnographic fieldwork by focusing on a pretty unusual case - floating churches, in Volgograd, Russia, more rural than urban, and definitely not the kind of instance you would run across in the mainstream innovation literature. The second case selection followed more or less the same oddly-informed pattern, this time - an on-land harbour, the brainchild of an experimental self-sufficient community recently established in Amsterdam, The Netherlands. 
Speaking from the field of Cultural Geography such an endeavour appears to be an opportune exercise, particularly for better understanding the underlying conditions of the current innovation ethos and the ways it (potentially) shapes future trajectories. The investigation draws on three main research questions, which address the meanings (1), workings (2) and expectations (3) connected to various innovation imaginaries, as follows:

(1) In what ways do different amphibious practices acknowledge the spatiotemporal and material conditions of innovation?

(2) How do those conditions enable the emergence of alternative innovations?

(3) To what extent are emergent alternatives influencing incumbent political repertoires as part of the current innovation ethos?

To answer these research questions, the dissertation brings into dialogue multiple disciplinary filiations and, as a secondary and more subtle objective, it reflects upon a new set of spatial (and temporal) imaginaries that would add up to the emergent spatial grammars currently animating geographical thought. Within the broader ambit of unpacking the workings of innovation processes, the theoretical and empirical exploration weaves contributions to the burgeoning strands of work on topological thinking, geographies of religion and secularism, archival practices and knowledge mobilities, urban progressive movements, and particularly, to the ongoing debates on new materialism. Consequently, the methodological sway of this study covers a spectrum ranging from grand theory to ethnographic accounts of micro-societal shifts.

The dissertation is structured into seven chapters and its red thread could be envisioned as describing a loop between chapters II and VI, accordingly entitled The Magic Mirror I and The Magic Mirror II. The second chapter provides a critical overview of grand innovation narratives and their diverse filiations across Western thought, to outline the conceptual imaginary that drives this investigation. The thematic focus of The Magic Mirror I concerns the normative distinction between innovation and imitation, which arguably deters an ampler understanding of innovation processes. Chapter III, The surface and the abyss, expands on this preliminary vision by resorting to an extensive genealogical exercise. Through a critical deployment of the surface/depth metaphor, it explores the catalytic potential of topological thinking to establish points of articulation between apparently opposed notions and canons of thought. Starting from a genealogy of mathematical developments and philosophical mediations toward the end point of geography, it addresses the interplay between the formal (axiomatic) and conceptual (problematic) dimensions of topology in suggesting some potentially alternative ways of re-imagining the role of topological thinking for spatial theory and human geography, and connecting these to the empirical exploration presented in chapter IV. 
Chapter IV explores the concept of creative reuse as an alternative modality to interrogate the materiality of things and their documentary sway beyond the immediate affordances dictated by circumstances of disposal or dissolution. Drawing on an ethnographic study of the Volga and Don riverscapes, it evokes the case of the floating churches built to support the revival of faith practices in the Volgograd oblast after the collapse of the Soviet Union. In attending to their impact in warping various temporal and geographical proximities, it suggests that their workings rely on topologies of fixed points and shifting spatialities, animated by forms of religious ritual and related creative manifestations. Through recourse to questions of materiality, mobility and affect it argues that creative reuse interventions provide productive ways of exposing and altering the residual surplus on which both things and processes of place-making rest upon.

Chapter $\mathrm{V}$ examines the role of creative reuse as an alternative imaginary specifically concerned with the residual surplus that results along dominant processes of accumulation and value production. In moving beyond circumstances of disposal or dissolution, it argues that creative reuse interventions provide inventive ways to exploit the productive latencies scored across incumbent sociotechnical arrangements. Building upon an ethnographic study of De Cewvel's on-land harbour, an experimental self-sufficient community recently established in Amsterdam, it shows how things that were otherwise redundant/disposed/forgotten can stimulate new material and affective dispositions that call into question established practices around sustainable, creative and inclusive city-making. Based on the findings, it goes on to suggest that creative reuse interventions enable new conditions of possibility for the enactment of alternative urban futures.

Chapter VI, The Magic Mirror II, closes the loop by connecting the findings to the introductory discussion from The Magic Mirror I, and elaborating further upon a more generous imaginary to tackle the workings of innovations, as well as the emergence of related alternatives. Thus, from the genealogical interrogation of topology to the unconventional interventions discussed in the empirical sections, creative reuse emerges as the vehicle of supprising returns. These enable a more generous reading that transcends the immediate affordances of mere imitation or circumstances of disposal - one that pivots on the key role of variation through mimesis or the potent afterlives of things and affects in animating alternative forms of innovation. The reference to alternatives should be understood both in relation to the dominant narrative of creative destruction, as well as to how various imaginaries - whether digested as secular, religious or otherwise become entangled and mirror each other in intriguing ways. Consequently, even when proceeding from the fairly basic distinction that things envisioned as fixed end up afloat and travelling around, as much as things expected to float and travel around become stranded, the idea of surprising returns opens a broad spectrum of meanings 
and potentialities. As such, the resulting instances expose realities that are much more turbulent than commonly asserted.

Chapter VII answers the main research questions and also grants recognition to creative reuse imaginaries as the inescapable complement to dominant processes of accumulation and value production. As such, the material and affective dispositions cultivated through the emergence of alternatives, within and between various practices, signal the dislocation work occasioned by processes of variation through mimesis. These emergent imaginaries rely on a logic of aspiration and differentiation, which allows them to interfere with, and shape each other, or even morph into new narratives of belonging and creative action. And this is usually achieved through a rather twisted symbiosis, one of peculiar association. The latter pertains to the loose/labile character of creative reuse imaginaries explored in the empirical chapters, which enables them to contract and expand under various readings. Somewhat paradoxically, their dynamics seems to mirror that of mainstream innovations through the performative re-enactment of conditions for success. However, they excel through the disposition for multiple entanglements that often defy the normative distinctions between formal and informal domains. This gives rise to broad fields of resonance in recasting all sorts of anamorphic reflections across the resulting amphibious domains of contingency. In other words, the more imaginaries they interfere with or even subsume, the higher chances become for innovative spin-offs. For a more synthetic overview of the findings, the last section of the chapter packs a final reflection in the form of some tentative corollaries inspired by this exploratory journey. 

Iulian Valentin (I.V.) Barba Lata

Wageningen School of Social Sciences (WASS)

Completed Training and Supervision Plan

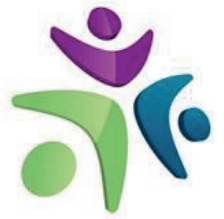

Wageningen School

of Social Sciences

\begin{tabular}{|c|c|c|c|}
\hline Name of the learning activity & Department/Institute & Year & ECTS* \\
\hline \multicolumn{4}{|l|}{ A) Project related competences } \\
\hline $\begin{array}{l}\text { Participatory Approaches in Planning, } \\
\text { Policy and Development }\end{array}$ & WASS & 2011 & 3 \\
\hline Human Geography UK, SAL-20406 & GEO/WUR & 2011 & 6 \\
\hline $\begin{array}{l}\text { Advanced Socio-spatial research } \\
\text { methodology (SAL-30806) }\end{array}$ & GEO/WUR & 2011 & 3 \\
\hline $\begin{array}{l}\text { Dis-locating innovation: The spatiotemporal } \\
\text { conditions of innovation in floating practices' }\end{array}$ & WASS Midterm review & 2012 & 1 \\
\hline 'Dis-locating innovation' & $\begin{array}{l}\text { Conference of Western Switzerland } \\
\text { Universities (CUSO) - } \\
\text { 'Representation and Non- } \\
\text { representation in Geography', } \\
\text { Geneva/ 'Journee Geographica } \\
\text { Helvetica', Fribourg }\end{array}$ & 2012 & 2 \\
\hline 'Retbinking topology' & $\begin{array}{l}\text { Association of American } \\
\text { Geographers Annual Meeting } \\
\text { (AAG), Tampa, United States }\end{array}$ & 2014 & 2 \\
\hline Research Internship & $\begin{array}{l}\text { Department of Geography - } \\
\text { Durham University }\end{array}$ & 2014 & 6 \\
\hline Presentations GEO PhD symposium & GEO/WUR & $2011-2015$ & 1 \\
\hline \multicolumn{4}{|l|}{ B) General research related competences } \\
\hline WASS Introduction course & WASS & 2011 & 1 \\
\hline $\begin{array}{l}\text { Risk Societies and Cosmopolitanism, } \\
\text { School of Critical Theory } 2012\end{array}$ & $\begin{array}{l}\text { Centre for the Humanities/ Utrecht } \\
\text { University }\end{array}$ & 2012 & 7.5 \\
\hline Lectures/seminars/debates & - & $2011-2015$ & 2 \\
\hline \multicolumn{4}{|c|}{ C) Career related competences/personal development } \\
\hline $\begin{array}{l}\text { WASS PhD council (member, chair and } \\
\text { board representative) }\end{array}$ & WASS & $2011-2013$ & 6 \\
\hline $\begin{array}{l}\text { Student supervision and teaching - } \\
\text { Landscape Economics and Politics } \\
\text { (AEP-22306) }\end{array}$ & AEP-GEO/WUR & 2015-2016 & 4 \\
\hline Total & & & 44.5 \\
\hline
\end{tabular}

*One credit according to ECTS is on average equivalent to 28 hours of study load 



\section{Funding}

This research was fully funded by the Wageningen School of Social Sciences (WASS), through a scholarship obtained during the WASS Open Round 2011. 

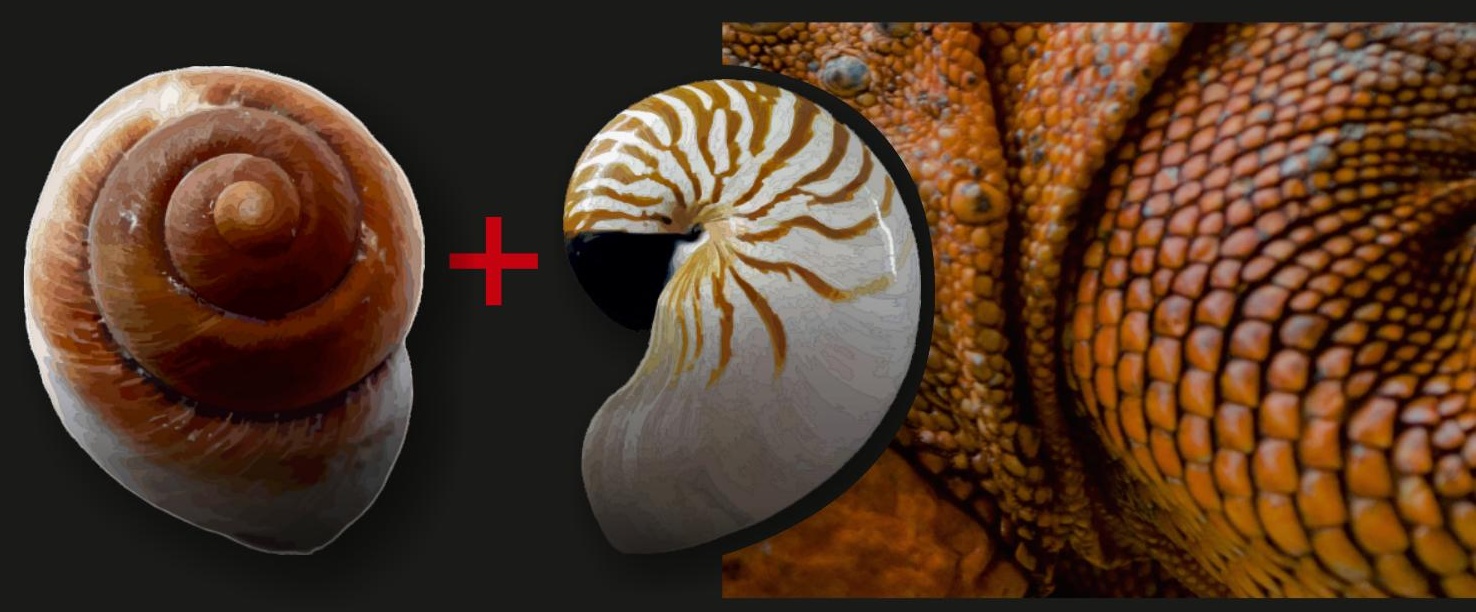